Cochrane Database of Systematic Reviews

\title{
Interventions for improving adherence to iron chelation therapy in people with sickle cell disease or thalassaemia (Review)
}

Fortin PM, Fisher SA, Madgwick KV, Trivella M, Hopewell S, Doree C, Estcourt LJ 


\section{TABLE OF CONTENTS}

HEADER

ABSTRACT

PLAIN LANGUAGE SUMMARY

SUMMARY OF FINDINGS FOR THE MAIN COMPARISON $\quad$. . . . . . . . . . . . . . . . . . . . . . $\quad 5$

BACKGROUND . . . . . . . . . . . . . . . . . . . . . . . . . . . . . . . . . . . . . . 7

OBJECTIVES . . . . . . . . . . . . . . . . . . . . . . . . . . . . . . . . . . . . . 99

METHODS . . . . . . . . . . . . . . . . . . . . . . . . . . . . . . . . . . . . . . 9

RESULTS . . . . . . . . . . . . . . . . . . . . . . . . . . . . . . . . . . . . . 14

Figure 1. . . . . . . . . . . . . . . . . . . . . . . . . . . . . . . 15

Figure 2. . . . . . . . . . . . . . . . . . . . . . . . . . . . . . . . . . . . . . 18

Figure 3. . . . . . . . . . . . . . . . . . . . . . . . . . . . . . . . . . . . . . 19

ADDITIONAL SUMMARY OF FINDINGS . . . . . . . . . . . . . . . . . . . . . . . . . . . . . . . . $\quad$. $\quad 27$

DISCUSSION . . . . . . . . . . . . . . . . . . . . . . . . . . . . . . . . . . . . . . . . 40

AUTHORS' CONCLUSIONS . . . . . . . . . . . . . . . . . . . . . . . . . . . . . . . . . . . . . . .

ACKNOWLEDGEMENTS . . . . . . . . . . . . . . . . . . . . . . . . . . . . . . . . . . . . . . 42

REFERENCES . . . . . . . . . . . . . . . . . . . . . . . . . . . . . . . . . . . . . . 42

CHARACTERISTICS OF STUDIES . . . . . . . . . . . . . . . . . . . . . . . . . . . . . . . . . . . . . . 49

DATA AND ANALYSES . . . . . . . . . . . . . . . . . . . . . . . . . . . . . . . . . . . . . . . . . . . . . . . . 101

ADDITIONAL TABLES . . . . . . . . . . . . . . . . . . . . . . . . . . . . . . . . . . . . . . . 104

CONTRIBUTIONS OF AUTHORS . . . . . . . . . . . . . . . . . . . . . . . . . . . . . . . . . . . . 106

DECLARATIONS OF INTEREST . . . . . . . . . . . . . . . . . . . . . . . . . . . . . . . . . . . . . .

SOURCES OF SUPPORT . . . . . . . . . . . . . . . . . . . . . . . . . . . . . . . . . . . . . . . . . . . . . . .

DIFFERENCES BETWEEN PROTOCOL AND REVIEW . . . . . . . . . . . . . . . . . . . . . . 107

INDEX TERMS . . . . . . . . . . . . . . . . . . . . . . . . . . . . . . . . . . . . . 108 
[Intervention Review]

\title{
Interventions for improving adherence to iron chelation therapy in people with sickle cell disease or thalassaemia
}

\author{
Patricia M Fortin ${ }^{1}$, Sheila A Fisher ${ }^{2}$, Karen V Madgwick ${ }^{3}$, Marialena Trivella ${ }^{4}$, Sally Hopewell ${ }^{5}$, Carolyn Doree ${ }^{2}$, Lise J Estcourt ${ }^{6}$ \\ ${ }^{1}$ Sechelt, Canada. ${ }^{2}$ Systematic Review Initiative, NHS Blood and Transplant, Oxford, UK. ${ }^{3}$ Department of Pathology, North \\ Middlesex University Hospital NHS Trust, London, UK. ${ }^{4}$ Centre for Statistics in Medicine, University of Oxford, Oxford, UK. \\ ${ }^{5}$ Nuffield Department of Orthopaedics, Rheumatology and Musculoskeletal Sciences (NDORMS), University of Oxford, Oxford, \\ UK. ${ }^{6}$ Haematology/Transfusion Medicine, NHS Blood and Transplant, Oxford, UK
}

Contact address: Lise J Estcourt, Haematology/Transfusion Medicine, NHS Blood and Transplant, Level 2, John Radcliffe Hospital, Headington, Oxford, OX3 9BQ, UK. lise.estcourt@nhsbt.nhs.uk, lise.estcourt@ndcls.ox.ac.uk.

Editorial group: Cochrane Cystic Fibrosis and Genetic Disorders Group.

Publication status and date: New, published in Issue 5, 2018.

Citation: Fortin PM, Fisher SA, Madgwick KV, Trivella M, Hopewell S, Doree C, Estcourt LJ. Interventions for improving adherence to iron chelation therapy in people with sickle cell disease or thalassaemia. Cochrane Database of Systematic Reviews 2018, Issue 5. Art. No.: CD012349. DOI: 10.1002/14651858.CD012349.pub2.

Copyright (C) 2018 The Cochrane Collaboration. Published by John Wiley \& Sons, Ltd.

\begin{abstract}
A B S T R A C T
Background

Regularly transfused people with sickle cell disease (SCD) and people with thalassaemia (who are transfusion-dependent or nontransfusion-dependent) are at risk of iron overload. Iron overload can lead to iron toxicity in vulnerable organs such as the heart, liver and endocrine glands; which can be prevented and treated with iron chelating agents. The intensive demands and uncomfortable side effects of therapy can have a negative impact on daily activities and well-being, which may affect adherence.
\end{abstract}

\section{Objectives}

To identify and assess the effectiveness of interventions (psychological and psychosocial, educational, medication interventions, or multi-component interventions) to improve adherence to iron chelation therapy in people with SCD or thalassaemia.

\section{Search methods}

We searched CENTRAL (the Cochrane Library), MEDLINE, Embase, CINAHL, PsycINFO, Psychology and Behavioral Sciences Collection, Web of Science Science \& Social Sciences Conference Proceedings Indexes and ongoing trial databases (01 February 2017). We searched the Cochrane Cystic Fibrosis and Genetic Disorders Group's Haemoglobinopathies Trials Register (12 December 2017).

\section{Selection criteria}

For trials comparing medications or medication changes, only randomised controlled trials (RCTs) were eligible for inclusion.

For studies including psychological and psychosocial interventions, educational Interventions, or multi-component interventions, nonRCTs, controlled before-after studies, and interrupted time series studies with adherence as a primary outcome were also eligible for inclusion.

\section{Data collection and analysis}

Three authors independently assessed trial eligibility, risk of bias and extracted data. The quality of the evidence was assessed using GRADE.

Interventions for improving adherence to iron chelation therapy in people with sickle cell disease or thalassaemia (Review)

Copyright () 2018 The Cochrane Collaboration. Published by John Wiley \& Sons, Ltd. 


\section{Main results}

We included 16 RCTs (1525 participants) published between 1997 and 2017. Most participants had $\beta$-thalassaemia major; 195 had SCD and 88 had $\beta$-thalassaemia intermedia. Mean age ranged from 11 to 41 years. One trial was of medication management and 15 RCTs were of medication interventions. Medications assessed were subcutaneous deferoxamine, and two oral-chelating agents, deferiprone and deferasirox.

We rated the quality of evidence as low to very low across all outcomes identified in this review.

Three trials measured quality of life (QoL) with validated instruments, but provided no analysable data and reported no difference in QoL.

\section{Deferiprone versus deferoxamine}

We are uncertain whether deferiprone increases adherence to iron chelation therapy (four trials, very low-quality evidence). Results could not be combined due to considerable heterogeneity (participants' age and different medication regimens). Medication adherence was high (deferiprone ( $85 \%$ to $94.9 \%)$; deferoxamine $(71.6 \%$ to $93 \%)$ ).

We are uncertain whether deferiprone increases the risk of agranulocytosis, risk ratio (RR) 7.88 ( $99 \%$ confidence interval (CI) 0.18 to 352.39); or has any effect on all-cause mortality, RR 0.44 (95\% CI 0.12 to 1.63 ) (one trial; 88 participants; very low-quality evidence).

\section{Deferasirox versus deferoxamine}

We are uncertain whether deferasirox increases adherence to iron chelation therapy, mean difference (MD) - 1.40 (95\% CI -3.66 to 0.86) (one trial; 197 participants; very-low quality evidence). Medication adherence was high (deferasirox (99\%); deferoxamine (100\%)). We are uncertain whether deferasirox decreases the risk of thalassaemia-related serious adverse events (SAEs), RR 0.95 ( $95 \%$ CI 0.41 to 2.17); or all-cause mortality, RR 0.96 (95\% CI 0.06 to 15.06) (two trials; 240 participants; very low-quality evidence).

We are uncertain whether deferasirox decreases the risk of SCD-related pain crises, RR 1.05 (95\% CI 0.68 to 1.62); or other SCDrelated SAEs, RR 1.08 (95\% CI 0.77 to 1.51 ) (one trial; 195 participants; very low-quality evidence).

\section{Deferasirox film-coated tablet (FCT) versus deferasirox dispersible tablet (DT)}

Deferasirox FCT may make little or no difference to adherence, RR 1.10 (95\% CI 0.99 to 1.22) (one trial; 173 participants; lowquality evidence). Medication adherence was high (FCT (92.9\%); DT (85.3\%)).

We are uncertain if deferasirox FCT increases the incidence of SAEs, RR 1.22 (95\% CI 0.62 to 2.37); or all-cause mortality, RR 2.97 (95\% CI 0.12 to 71.81 ) (one trial; 173 participants; very low-quality evidence).

\section{Deferiprone and deferoxamine combined versus deferiprone alone}

We are uncertain if deferiprone and deferoxamine combined increases adherence to iron chelation therapy (very low-quality evidence). Medication adherence was high (deferiprone $92.7 \%$ (range $37 \%$ to $100 \%$ ) to $93.6 \%$ (range $56 \%$ to $100 \%$ ); deferoxamine $70.6 \%$ (range $25 \%$ to $100 \%$ ).

Combination therapy may make little or no difference to the risk of SAEs, RR 0.15 (95\% CI 0.01 to 2.81) (one trial; 213 participants; low-quality evidence).

We are uncertain if combination therapy decreases all-cause mortality, RR 0.77 (95\% CI 0.18 to 3.35) (two trials; 237 participants; very low-quality evidence).

\section{Deferiprone and deferoxamine combined versus deferoxamine alone}

Deferiprone and deferoxamine combined may have little or no effect on adherence to iron chelation therapy (four trials; 216 participants; low-quality evidence). Medication adherence was high (deferoxamine $91.4 \%$ to $96.1 \%$; deferiprone: $82.4 \%$ )

Deferiprone and deferoxamine combined, may have little or no difference in SAEs or mortality (low-quality evidence). No SAEs occurred in three trials and were not reported in one trial. No deaths occurred in two trials and were not reported in two trials.

\section{Deferiprone and deferoxamine combined versus deferiprone and deferasirox combined}

Deferiprone and deferasirox combined may improve adherence to iron chelation therapy, RR 0.84 (95\% CI 0.72 to 0.99 ) (one trial; 96 participants; low-quality evidence). Medication adherence was high (deferiprone and deferoxamine: 80\%; deferiprone and deferasirox: $95 \%)$.

Interventions for improving adherence to iron chelation therapy in people with sickle cell disease or thalassaemia (Review) 
We are uncertain if deferiprone and deferasirox decreases the incidence of SAEs, RR 1.00 (95\% CI 0.06 to 15.53) (one trial; 96 participants; very low-quality evidence).

There were no deaths in the trial (low-quality evidence).

\section{Medication management versus standard care}

We are uncertain if medication management improves health-related QoL (one trial; 48 participants; very low-quality evidence). Adherence was only measured in one arm of the trial.

\section{Authors' conclusions}

The medication comparisons included in this review had higher than average adherence rates not accounted for by differences in medication administration or side effects.

Participants may have been selected based on higher adherence to trial medications at baseline. Also, within the clinical trial context, there is increased attention and involvement of clinicians, thus high adherence rates may be an artefact of trial participation.

Real-world, pragmatic trials in community and clinic settings are needed that examine both confirmed or unconfirmed adherence strategies that may increase adherence to iron chelation therapy.

Due to lack of evidence this review cannot comment on intervention strategies for different age groups.

\section{PLAIN LANGUAGE SUMMARY}

\section{Strategies to increase adherence to iron chelation therapy in people with sickle cell disease or thalassaemia}

\section{Review question}

We wanted to determine if there are any interventions (medication, psychological or educational) that would help people adhere to their iron chelation therapy.

\section{Background}

People with sickle cell disease or thalassaemia who receive regular transfusions, are exposed to iron overload which can result in toxicity to organs and death. Iron chelation therapy is used to prevent or treat iron overload, but it can be a demanding regimen, and have unwanted side effects. There are three types of iron chelators being used to treat iron overload: deferoxamine given subcutaneously (by injecting a drug into the tissue layer between the skin and the muscle); and two agents that are taken orally, deferiprone and deferasirox.

\section{Search date}

The evidence is current to 12 December 2017.

\section{Study characteristics}

We searched the literature for both randomised and non-randomised studies, and found 16 randomised trials with 1525 participants, published between 1997 and 2017. Most people had $\beta$-thalassaemia major; one trial included people with SCD and one included people with a milder form of thalassaemia (thalassaemia intermedia). Mean age ranged from 11 years to 41 years. We included one trial of medication management and 15 trials comparing different drug treatments.

\section{Key results}

Trials included comparisons of individual agents to each other or a combination of drugs compared to one drug alone or to other combinations of drugs.

We were uncertain if single agents or combined agents made any difference in adherence rates, serious adverse events or mortality. Quality of life, measured using validated questionnaires, was only reported in two trials, but not enough data were reported to determine any differences between treatments.

There was no evidence on intervention strategies for different age groups.

Interventions for improving adherence to iron chelation therapy in people with sickle cell disease or thalassaemia (Review)

Copyright () 2018 The Cochrane Collaboration. Published by John Wiley \& Sons, Ltd. 
We found that there was an unusually high adherence rate to all drugs and combinations of drugs in all the trials. This may be because participants may have been selected based on their ability to stick to medication regimens. Also, adherence may increase in trial participants when there is a higher level of clinician involvement in care.

We concluded that real-world randomised and non-randomised trials, run in both the community and in clinics, are needed to examine a variety of proven and unproven strategies that may be useful for increasing adherence to iron chelation therapy.

\section{Quality of evidence}

We rated the quality of evidence as low to very low across all of the outcomes in this review. This was due to trials being at serious or very serious risk of bias; outcome estimates being imprecise (wide confidence intervals); and not widely applicable (with some trials conducted only in children of a specific age and meeting specific criteria). 


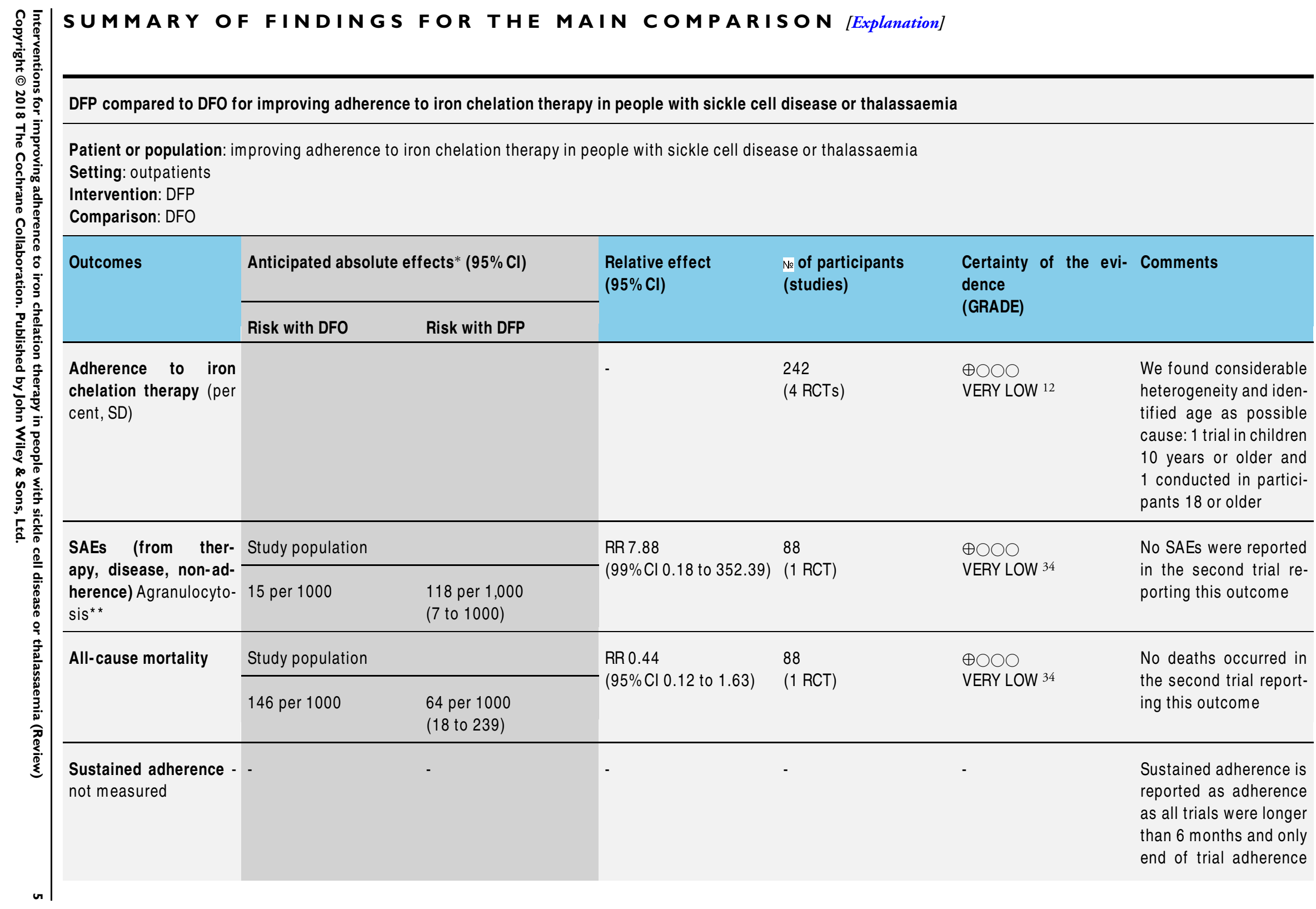


ported

${ }^{*}$ The risk in the intervention group (and its $95 \%$ confidence interval) is based on the assumed risk in the comparison group and the relative effect of the intervention (and its $95 \% \mathrm{Cl})$.

Cl: confidence interval; DFO: deferoxamine; DFP: deferiprone; RCT: randomised controlled trial; RR: risk ratio; SD: standard deviation.

\section{GRADE Working Group grades of evidence}

High certainty: we are very confident that the true effect lies close to that of the estimate of the effect

Moderate certainty: we are moderately confident in the effect estimate: the true effect is likely to be close to the estimate of the effect, but there is a possibility that it is substantially different

Low certainty: our confidence in the effect estimate is limited: the true effect may be substantially different from the estimate of the effect

Very low certainty: we have very little confidence in the effect estimate: the true effect is likely to be substantially different from the estimate of effect

${ }^{1}$ We downgraded the quality of evidence by 1 for risk of bias due to high or uncertain risk of bias due to lack of blinding of participants and personnel in all four RCTs, as well as selection bias (Olivieri 1997), attrition bias (El Beshlawy 2008;

Olivieri 1997), reporting bias (El Beshlawy 2008; Pennell 2006), and other bias (Pennell 2006).

${ }^{2}$ We downgraded the quality of evidence by 2 for inconsistency due to considerable heterogeneity in comparison.

${ }^{3}$ We downgraded the quality of evidence by 2 for imprecision due to very wide Cls that included clinically important benefits and harms.

${ }^{4}$ We downgraded the quality of evidence by 1 for indirectness as the trial was conducted in participants with thalassaemia intermedia only; a milder form of thalassaemia

** Risk estimate based on: Tricta F, Uetrecht J, Galanello R, et al. Deferiprone-induced agranulocytosis: 20 years of clinical observations. American Journal of Hematology. 2016;91(10):1026-1031. doi:10.1002/ajh.24479. 


\section{B A C K G R O U N D}

\section{Description of the condition}

Haemoglobinopathies are a range of inherited disorders resulting from mutations of the globin genes (the protein component of haemoglobin). Two of the most common of these disorders are sickle cell disease (SCD) and thalassaemia.

\section{Sickle cell disease}

SCD is an inheritable blood disorder, which can lead to life-threatening complications. People with SCD experience episodes of severe pain, and other complications including anaemia, end-organ damage, pulmonary complications, kidney disease, and increased susceptibility to infections and stroke (Pleasants 2014). It is one of the most common severe monogenic disorders in the world, due to the inheritance of two abnormal haemoglobin (beta globin) genes (Rees 2010). Populations originating from sub-Saharan Africa, Spanish-speaking regions in the western hemisphere (South America, the Caribbean, and Central America), the Middle East, India and parts of the Mediterranean are predominantly affected. Reductions in infant and child mortality and increasing migration from highly affected countries have made this a worldwide problem (Piel 2012). Over 12,500 people in the UK and 100,000 in the USA suffer from the disease (NICE 2010; Pleasants 2014).

The term SCD refers to all mutations that cause the disease, of which there are three main types. Sickle cell anaemia is the most common form of the disease (up to $70 \%$ of cases of SCD in people of African origin) and is due to the inheritance of two beta globin $\mathrm{S}(\beta \mathrm{S})$ alleles (haemoglobin $(\mathrm{Hb}) \mathrm{SS}$ ). The second most common genotype (up to $30 \%$ of cases in people of African origin) is haemoglobin SC disease (HbSC disease) and is due to the co-inheritance of the $\beta \mathrm{S}$ and $\beta \mathrm{C}$ alleles; this tends to be a more moderate form of the disease. The third major type of SCD occurs when $\beta \mathrm{S}$ is inherited with a $\beta$-thalassaemia allele, causing $\mathrm{HbS} / \beta$-thalassaemia (Rees 2010). People who have inherited a thalassaemia null mutation $\left(\mathrm{HbS} \beta^{\circ}\right)$ have a disease that is clinically indistinguishable from sickle cell anaemia, whereas people with $\mathrm{HbS} \beta^{+}$ thalassaemia have a milder disorder. In high-income nations, people with SCD are expected to live into their 40s, 50 s and beyond; whereas in low-income countries, including some African nations, it is estimated that between $50 \%$ to $90 \%$ of children born with HbSS die before their fifth birthday (Gravitz 2014; Grosse 2011). Red blood cell transfusions can be given to treat complications of SCD (e.g. acute chest syndrome), this often involves a single transfusion episode, or they can be part of a regular long-term transfusion programme to prevent complications of SCD such as stroke in children (Yawn 2014).

\section{Thalassaemia}

The term thalassaemia describes a group of inheritable disorders caused by the absence or reduction in globin chain production. This results in ineffective red blood cell production, anaemia and poor oxygen delivery. The genetic defect can be in the $\alpha$ or $\beta$ globin chain ( $\alpha$-thalassaemia, $\beta$-thalassaemia or $\mathrm{H}$ disease). In $\beta$ thalassaemia, reduced or absent $\beta$ globulin production leads to an excess of free $\alpha$-globin chains resulting in severe anaemia and bone marrow hyperplasia (abnormal cell growth) preventing normal development. In $\mathrm{H}$ disease and $\alpha$-thalassaemia, the $\alpha$-globin chains are affected and disease can vary from mild (where reduced, but adequate, amounts of the functional globin chains are produced) to severe (where no effective haemoglobin is produced) (UK Thalassaemia Society 2008). Complications that may occur include infections, bone diseases, enlarged spleen, slowed growth rates, cardiomyopathy, venous thrombosis, pulmonary hypertension, and hypothyroidism (Rund 2005).

Thalassaemia is common in people from the Mediterranean, the Middle East, Southeast Asia, the Indian subcontinent, and Africa (Piel 2014; UK Thalassaemia Society 2008). It is estimated that there are over 1000 people with thalassaemia in the UK (APPG 2009). In high-income countries most affected children survive with a chronic disorder; however, most children born with thalassaemia are in low-income countries die before the age of five years (Modell 2008). Nevertheless, the thalassaemias are a global health burden due to population migration and growth and improved survival leading to an increase in the incidence of the disorder (Piel 2014).

Regular red blood cell transfusion is the standard treatment to correct anaemia and to enable growth and development, normal activities and to inhibit bone marrow expansion. People with severe forms, $\beta$-thalassaemia major, require life-long transfusions from the first year of life.

\section{Iron chelation therapy and adherence}

Regularly transfused people with SCD, as well as transfusiondependent, and non-transfusion-dependent people with thalassaemia, are exposed to transfusion-related iron overload. Transfusion-related iron overload can lead to iron toxicity, with organs such as the heart, liver and endocrine glands being particularly vulnerable. Iron overload is the major cause of morbidity and mortality in thalassaemia (Aydinok 2014; Rund 2005; Trachtenberg 2012).

Iron chelating agents are used for preventing and treating iron overload. Deferoxamine (DFO) has been the standard treatment for the last 40 years; it is administered subcutaneously or intravenously usually over eight to 12 hours, up to seven days a week. More recently two oral chelating agents, deferiprone (DFP) and then deferasirox (DFX), have been licensed. These were initially introduced as second-line agents in children six years and older with $\beta$-thalassaemia major, or in people when DFO is contraindi- 
cated or found to be inadequate (Fisher 2013). These oral agents are becoming more commonly used, particularly DFX, because of the ease of administration compared to subcutaneous or intravenous DFO (Aydinok 2014).

Licensed iron chelating agents are effective at iron removal; however, the treatment is not without side effects (Telfer 2006). Side effects with DFO include pain or skin reactions at the injection site, retinal toxicity and hearing loss. Side effects with DFX include skin rashes, gastroenteritis, an increase in liver enzymes, and reduced kidney function. Adverse events reported in people taking DFP include gastrointestinal disturbances, arthropathy (joint disease), raised liver enzymes, neutropenia (a decrease in neutrophils, a type of white blood cell, in the blood stream) and agranulocytosis (lowered white blood cell count). Regular blood sampling is recommended to monitor neutropenia, renal function and liver enzymes in people taking oral chelating agents (Fisher 2013).

Adherence to medications is defined as the extent to which a person's use of the medicine matches the agreed prescription from the healthcare provider (NICE 2009; Walsh 2014). Moderate adherence is defined as taking $60 \%$ to $80 \%$ of a prescribed dose, while high adherence can include the continued use of the medicine or taking at least $80 \%$ of the recommended dose. There are several ways to measure adherence including the self-reporting of medication use or more objective factors such as pill counts, prescription refills, urinary assays or in the case of iron chelation, signs of iron overload (Ryan 2014; Walsh 2014). Adherence rates can vary widely, a recent review reported that adherence rates to the iron chelator deferasirox ranged between 22\% and 89\% (Loiselle 2016).

Research suggests that iron chelation therapies impact on a person's quality of life (QoL) and result in low levels of personal satisfaction. The intensive demands and uncomfortable side effects of iron chelation therapy can have a negative impact on daily activities and well-being, which may affect adherence to therapy (Abetz 2006; Payne 2008; Rofail 2010). Other factors affecting adherence to medications include inappropriate use, the quality of information provided to the individual, complex treatment regimens, as well as intolerance to the harms caused by the medications (Ryan 2014). Non-adherence can be both intentional and unintentional, with intentional non-adherence being influenced by such factors as poor communication, adverse effects, personal preferences or beliefs and disagreement with the need for treatment; whereas unintentional non-adherence is influenced by factors generally beyond the person's control such as forgetfulness or difficulties in understanding instructions (NICE 2009; Ryan 2014; Trachtenberg 2012). Sub-optimal adherence can increase adverse events associated with iron overload and result in increased cost of care, hospitalisations, and severe morbidity and mortality (Payne 2008; Vekeman 2016; WHO 2003).

\section{Description of the intervention}

The research on adherence and appropriate use of medicines is vast and complex and comprises a number of studies targeting people taking the medication, clinicians, indications and specific classes of medications. This research has also been reviewed in many systematic reviews as well as overviews of systematic reviews and in guidelines (Costello 2004; NCCPC 2009; NICE 2009; Ryan 2014; WHO 2003).

For this review we focus on the individual with SCD or thalassaemia, with interventions to increase adherence to iron chelation therapy being divided into three main categories. These are psychological and psychosocial interventions, educational interventions and medication interventions. These interventions may be delivered alone or in combination (as a complex intervention). For instance, combining psychological with psychosocial interventions such as symptom self-management with peer support; or medication changes implemented with reconciliation strategies or complemented with medication information and education.

\section{Psychological and psychosocial interventions}

Psychological and psychosocial therapies that may promote medication adherence include interventions to promote behavioural change such as cognitive behavioural therapy (CBT), as well as peer support, counselling and skills development (communication, social, emotional). In addition there is an increasing emphasis on health-system interventions that may influence adherence such as patient-centred care and shared decision-making (NCCPC 2009; Ryan 2014; WHO 2003).

In an outpatient clinic survey of 328 people with SCD using the Patient Health Questionnaire 9, up to $60 \%$ of people with SCD experienced mild to severe depressive symptoms. Interventions to address depression and other co-morbidities may promote medication adherence, and depending on the degree of depression or other co-morbidities can include medications, guided self-help, individual or group CBT or peer support (NCCMH 2010; NICE 2009; Thomas 2013).

\section{Education interventions}

Educational interventions may include disease and medication information, and assistance with communication skills to facilitate communication with healthcare providers (Haywood 2009; Ryan 2014). Interventions in the form of personal communication, structured presentations, and formal educational activities delivered by clinicians or non-medical personnel are included in this category.

\section{Medication interventions}

The identification and correction of medication issues such as under-utilisation, dosing and scheduling, allergies and contraindications, financial issues and inadequate monitoring may impact on adherence and health outcomes. Additional strategies such as 
positive medication changes to reduce burden or increase effectiveness, route of administration, risk minimisation and medication reconciliation may be used to promote improved medication adherence (NCCPC 2009; Ryan 2014).

\section{How the intervention might work}

\section{Psychological and psychosocial interventions}

People with chronic illness face a variety of psychological and psychosocial problems including depression, anxiety disorders, disease burden and restrictions on social and occupational functioning. Research suggests that skill development to help people with chronic illnesses cope with adverse effects of medication and any co-morbidities will decrease disease burden, and improve their health-related QoL (NCCMH 2010; NCCPC 2009). The use of cognitive aids, clear instructions and realistic expectations can improve adherence (Wertheimer 2003). Person-centred psychological and psychosocial interventions encourage self-management skills, shared decision-making and self-efficacy (NCCPC 2009; NICE 2009).

\section{Educational interventions}

Tailored educational interventions can be delivered to individuals or groups and can be delivered face-to-face or remotely. Educational interventions may include both a simple approach, such as evidence-based plain language information, by written or verbal communication, or a multi-faceted approach that considers the wider environment, management, decision making, lifestyle and communication roles taken on by the person taking the medication (Ryan 2014). Each approach should be tailored to the individual (NCCPC 2009; WHO 2003).

\section{Medication interventions}

Iron levels are monitored in people receiving regular transfusions. An increasing iron burden may necessitate medication changes or more aggressive iron chelation therapy such as increasing doses or combination therapy. People may also change medications multiple times due to worsening iron overload, side effects, or personal preferences (Trachtenberg 2014). Medication changes that reflect personal preferences or minimise harms and improve outcomes, combined with medication reconciliation strategies including audit and feedback, prescription and medication help lines, counselling and age-appropriate discharge instructions, may help to address and improve adherence (NCCPC 2009; Ryan 2014). Medication interventions also include medication management which is a person-centred intervention by a clinician (often a pharmacist) to optimise drug therapy in order to improve outcomes for the person (American Pharmacists Association 2008).

\section{Why it is important to do this review}

Adherence to iron chelation therapy is necessary to decrease the risk of morbidity and mortality associated with iron overload. Poor adherence can also result in increased healthcare costs. It is therefore important to understand the effectiveness and limitations of interventions which can be used to influence adherence in people receiving iron chelation therapy for SCD or thalassaemia.

\section{O B J E C T I VES}

To identify and assess the effectiveness of interventions to improve adherence to iron chelation therapy compared to standard care in people with SCD or thalassaemia including:

1. identifying and assessing the effectiveness of different types of interventions (psychological and psychosocial, educational, medication interventions (which include comparisons of adherence between different iron chelators), or multi-component interventions);

2. identifying and assessing the effectiveness of interventions specific to different age groups (children, adolescents, adults).

\section{METHODS}

\section{Criteria for considering studies for this review}

\section{Types of studies}

We included randomised controlled trials (RCTs) comparing one or more adherence interventions, to standard care.

For studies comparing medications or medication changes, we only included RCTs.

If no RCTs were available, we planned to include non-randomised studies of interventions (NRSIs), controlled before-after (CBA) studies, and interrupted time series (ITS) studies including repeated measures designs for those studies including psychological and psychosocial interventions, educational Interventions, or multi-component interventions. We used the Cochrane Effective Practice and Organisation of Care (EPOC) Group's definition of study designs to consider studies for inclusion (EPOC 2015).

We planned to include cluster-randomised trials, non-randomised cluster trials, and CBA studies if they had at least two intervention sites and two control sites. We excluded cluster-randomised trials, non-randomised cluster trials, and CBA studies that had only one intervention or control site because the intervention (or comparison) may be confounded by study site making it difficult to attribute any observed differences to the intervention rather than to other site-specific variables (EPOC 2015). 
We planned to include ITS and repeated measures studies which had a clearly defined point in time when the intervention occurred and at least three data points before and after the intervention. We excluded ITS studies that did not have a clearly defined point in time when the intervention occurred, or fewer than three data points before and after the intervention, or the ITS study ignored secular (trend) changes, performed a simple t-test of the pre- versus post-intervention periods and re-analysis of the data was not possible (in accordance with EPOC 2015 recommendations).

\section{Types of participants}

Children, adolescents, or their caregivers, and adults with SCD or transfusion-dependent or non-transfusion-dependent thalassaemia.

\section{Types of interventions}

- Psychological and psychosocial Interventions

- Educational interventions

- Medication interventions

- Multi-component interventions (combining aspects of the above interventions)

\section{versus}

- Standard care (as defined in the trial)

\section{Types of outcome measures}

\section{Primary outcomes}

1. Adherence to iron chelation therapy rates (defined as per cent of doses administered (number of doses of the iron chelator taken, out of number prescribed), measured for a minimum of three months

2. Serious adverse events (SAEs) (including complications from the therapy, the disease itself, and non-adherence to chelation therapy)

\section{All-cause mortality}

We categorised all-cause mortality and SAEs according to short, medium-, and long-term outcomes. We reported the exact definition of these time frames over time periods that are common to as many trials as possible (e.g. zero to one year, one to five years, over five years).

\section{Secondary outcomes}

1. Sustained adherence to therapy (measured for a minimum of six months)

2. Health-related QoL (as measured by validated instruments)

3. Iron overload (defined by ferritin over $1000 \mu \mathrm{g} / \mathrm{L}$, or clinical symptoms, or signs of iron overload, e.g. magnetic resonance imaging (MRI) T2* cardiac iron content, MRI R2* liver iron content, liver biopsy, or the need for medically indicated additional or change in chelation therapy)

4. Organ damage (including cardiac failure, endocrine disease, surrogate markers of organ damage (creatinine), histologic evidence of hepatic fibrosis)

5. Other adverse events related to iron chelation

We categorised health-related QoL, iron overload and organ damage according to short-, medium-, and long-term outcomes. We reported the exact definition of these time frames over time periods that are common to as many studies as possible (e.g. up to six months, six to 12 months, over 12 months).

\section{Search methods for identification of studies}

We searched for all relevant published and unpublished trials without restrictions on language, year or publication status.

\section{Electronic searches}

We identified studies from the Cochrane Cystic Fibrosis and Genetic Disorders Group's Haemoglobinopathies Trials Register using the terms: (sickle cell OR thalassaemia) AND iron chelation. The Haemoglobinopathies Trials Register is compiled from electronic searches of the Cochrane Central Register of Controlled Trials (CENTRAL) (updated each new issue of the Cochrane Library) and weekly searches of MEDLINE. Unpublished work is identified by searching the abstract books of five major conferences: the European Haematology Association conference; the American Society of Hematology conference; the British Society for Haematology Annual Scientific Meeting; the Caribbean Public Health Agency Annual Scientific Meeting (formerly the Caribbean Health Research Council Meeting); and the National Sickle Cell Disease Program Annual Meeting. For full details of all searching activities for the register, please see the relevant section of the Cochrane Cystic Fibrosis and Genetic Disorders Group's website.

Date of the most recent search of the Cochrane Cystic Fibrosis and Genetic Disorders Group's Haemoglobinopathies Trials Register: 12 December 2017.

In addition to the above, we conducted a search of the following databases to include RCTs, NRSIs, CBA and ITS studies:

- Cochrane Central Register of Controlled Trials (CENTRAL; 2017, Issue 1) and Other Reviews (DARE; 2015 Issue 2) (www.cochranelibrary.com/) searched 01 February 2017;

- PubMed ( Epub Ahead of Print, In-Process and Other Non-Indexed Citations, for recent records not yet added to MEDLINE) (www.ncbi.nlm.nih.gov/sites/entrez) searched 01 February 2017;

- MEDLINE (OvidSP, Epub Ahead of Print, In-Process and Other Non-Indexed Citations, Ovid MEDLINE Daily and Ovid MEDLINE, 1946 to 01 February 2017);

- Embase (OvidSP, 1974 to 01 February 2017); 
- CINAHL (EBSCOHost, 1937 to 01 February 2017);

- PsycINFO (EBSCOHost, 1900 to 01 February 2017);

- ProQuest Dissertations \& Theses Global (ProQuest, 1861

to 01 February 2017);

- Psychology and Behavioral Sciences Collection

(EBSCOHost, 1930 to 01 February 2017);

- Web of Science Science \& Social Sciences Conference Proceedings Indexes (CPSI-S \& CPSSI, 1990 to 01 February 2017).

We also searched the following trial registries for ongoing trials:

- ClinicalTrials.gov ( clinicaltrials.gov/) searched on 01

February 2017;

- WHO International Clinical Trials Registry Platform (

ICTRP) ( apps.who.int/trialsearch/) searched on 01 February 2017;

- ISRCTN registry ( www.isrctn.com/) searched on 01

February 2017.

Search strategies can be found in an appendix (Appendix 1).

\section{Searching other resources}

We handsearched reference lists of included trials in order to identify further relevant trials.

\section{Data collection and analysis}

\section{Selection of studies}

We selected trials according to chapter 7 of the Cochrane Handbook for Systematic Reviews of Interventions (Higgins 2011b). Three review authors (PF, KM, LE) independently screened all electronically-derived citations and abstracts of papers identified by the search strategy for relevance. We excluded studies that were clearly irrelevant at this stage based on the abstract. Three review authors (PF, KM, LE) independently assessed the full texts of all potentially-relevant studies for eligibility against the criteria outlined above. We resolved disagreements by discussion, if we did not reach a consensus or if we were unsure of trial eligibility, we consulted a third review author (LE or SH). We sought further information from trial investigators if the trial report or abstract contained insufficient data to make a decision about eligibility. We used Covidence software to assess trial eligibility, which included ascertaining whether the participants had SCD or thalassaemia, if the trial addressed interventions to improve adherence to iron chelation therapy, and whether the trial was randomised or a NRSI or a CBA or an ITS study (Covidence). We recorded the reasons why potentially-relevant studies failed to meet the eligibility criteria.

\section{Data extraction and management}

Three review authors (PF, SF, KM) extracted the data according to Cochrane guidelines (Higgins 2011a). We resolved disagreements by consensus or we consulted a fourth review author (LE). We extracted data independently for all of the trials using Covidence modified to reflect the outcomes in this review (Covidence). In addition, we used the available tables in Review Manager 5 to extract data on trial characteristics as below (RevMan 2014).

\section{General information}

Review author's name, date of data extraction, study ID, first author of study, author's contact address (if available), citation of paper, objectives of the study.

\section{Study details}

Design, location, setting, sample size, power calculation, treatment allocation, inclusion and exclusion criteria, reasons for exclusion, comparability of groups, length of follow-up, stratification, stopping rules described, statistical analysis, results, conclusion, and funding.

\section{Characteristics of participants}

Age, gender, total number recruited, total number randomised, total number analysed, types of underlying disease, loss to followup numbers, dropouts (percentage in each arm) with reasons, protocol violations, iron chelating agent, previous treatments, current treatment, prognostic factors, co-morbidities, ferritin levels.

\section{Interventions}

Details of the interventions including type of intervention whether psychological and psychosocial or educational or medication or multi-component interventions, how the intervention is being delivered (i.e. group, face-to-face, written information, electronically) and by whom (i.e. clinicians, peers) and where the intervention is being delivered (i.e. hospital, clinic, home).

\section{Outcomes measured}

Adherence rates, SAEs, all-cause mortality, sustained adherence to therapy, health-related QoL, iron overload defined by ferritin over $1000 \mu \mathrm{g} / \mathrm{L}$ or clinical symptoms or signs of iron overload or need for medically indicated additional or change in chelation therapy (or any combination of these), evidence of organ damage, other adverse events.

We used both full-text versions and abstracts as data sources and used one data extraction form for each unique study. Where sources did not provide sufficient information, we contacted authors for additional details.

Three review authors (PF, SF, KM) entered data and we resolved disagreements by consensus. 
If NRSIs had been identified we planned to extract data according to the criteria developed for NRSIs as recommended in Chapter 13 of the Cochrane Handbook of Systematic Reviews of Interventions (Reeves 2011). In addition to the items above, for NRSIs, CBA and ITS studies we also planned to collect data on: confounding factors; the comparability of groups on confounding factors; methods used to control for confounding and on multiple effect estimates (both unadjusted and adjusted estimates) as recommended in chapter 13 of the Cochrane Handbook of Systematic Reviews of Interventions (Reeves 2011).

\section{Assessment of risk of bias in included studies}

Three review authors (PF, KM, SF) assessed all included trials for possible risks of bias as described in the Cochrane Handbook of Systematic Reviews of Interventions (Higgins 2011c).

The assessment included information about the design, the conduct and the analysis of the trial. We assessed each criterion using Cochrane's tool for assessing the risk of bias for RCTs (classed as 'low', 'high' or 'unclear' risk) in the following areas.

- Selection bias (random sequence generation and allocation concealment)

- Performance bias (blinding of participants and personnel)

- Detection bias (blinding of outcome assessment)

- Attrition bias (incomplete outcome data)

- Reporting bias (selective reporting)

- Other bias

We resolved disagreements on the assessment of quality of an included trial by discussion until we reached consensus or failing that by consulting a fourth review author (LE).

The only included trials were RCTs. In future updates of this review, we plan to use the ROBINS-I tool (Risk Of Bias In Nonrandomized Studies of Interventions) to rate the quality of NRSIs and CBA studies (Sterne 2016). The tool, uses signalling questions and covers seven domains (listed below) where the quality of evidence is rated as 'low', 'moderate', 'serious', 'critical' or 'no information'. Please refer to an appendix for a copy of the tool (Appendix 2).

- Bias due to confounding

- Bias in the selection of participants

- Bias in measurement of interventions

- Bias due to departure from intended interventions

- Bias due to missing data

- Bias in measurement of outcomes

- Bias in the selection of the reported result

In future updates of this review, for ITS studies we plan to use the risk of bias criteria below as suggested for EPOC reviews (EPOC 2015).

- Was the intervention independent of other changes?

- Was the shape of the intervention effect pre-specified?

- Was the intervention unlikely to affect data collection?
- Was knowledge of the allocated interventions adequately prevented during the study?

- Were incomplete outcome data adequately addressed?

- Was the study free from selective outcome reporting?

- Was the study free from other risks of bias?

\section{Measures of treatment effect}

\section{RCTs}

For RCTs of continuous outcomes we recorded the mean, standard deviation (SD) and total number of participants in both the treatment and control groups. For those using the same scale, we performed analyses using the mean difference (MD) with $95 \%$ confidence intervals (CIs); for those reported using different scales, we would have used standardised mean difference (SMD).

For RCTs of dichotomous outcomes we recorded the number of events and the total number of participants in both the treatment and control groups and reported the pooled risk ratio (RR) with a 95\% CI (Deeks 2011). Where the number of observed events is small (less than $5 \%$ of sample per group), and where trials have balanced treatment groups, we would have reported the Peto odds ratio (OR) with 95\% CI (Deeks 2011).

There were no eligible cluster randomised trials, if such trials are included in future updates of this review, we plan to extract and report direct estimates of the effect measure (e.g. RR with a 95\% CI) from an analysis that accounts for the clustered design. We will obtain statistical advice (MT) to ensure the analysis is appropriate. If appropriate analyses are not available, we will make every effort to approximate the analysis following the recommendations in chapter 16 of the Cochrane Handbook for Systematic Reviews of Interventions (Higgins 2011d).

\section{Non-randomised studies}

There were no eligible NRSIs, if such studies are included in future updates of this review, we plan to extract and report the RR with a 95\% CI for dichotomous outcomes, adjusting for baseline differences (such as Poisson regressions or logistic regressions) or the ratio of RRs (i.e. the RR post intervention / RR pre intervention). For continuous variables we will extract and report the absolute change from a statistical analysis adjusting for baseline differences (e.g. regression models, mixed models or hierarchical models) or the relative change adjusted for baseline differences in the outcome measures (i.e. the absolute post-intervention difference between the intervention and control groups, as well as the absolute pre-intervention difference between the intervention and control groups / the post-intervention level in the control group) (EPOC 2015). 


\section{ITS studies}

There were no eligible ITS studies, if such studies are included in future updates, we plan to standardise data by dividing the level (or time slope) and standard error (SE) by the SD of the preintervention slope, in order to obtain the effect sizes.

Where appropriate, we plan to report the number-needed-totreat-to-benefit (NNTB) and the number-needed-to-treat-toharm (NNTH) with CIs.

If we are unable to report the available data in any of the formats described above, we will perform a narrative report, and if appropriate, present the data in tables.

\section{Unit of analysis issues}

For trials with multiple treatment groups or interventions, we included subgroups that were considered relevant to the analysis. If appropriate, we combined groups to create a single pair-wise comparison. If this was not possible, we selected the most appropriate pair of interventions and excluded the others (Higgins 2011d). No trials randomised participants more than once.

There were no included cluster randomised studies or NRSIs. If these are included in future updates of this review, we plan to treat any unit of analysis issues that arise in accordance with the advice given in chapter 16 of the Cochrane Handbook for Systematic Reviews of Interventions (Higgins 2011d). .

There were no included ITS studies. If these are included in future updates of this review, we plan to deal with any unit of analysis issues arising from their inclusion according to the EPOC recommendations (EPOC 2015).

\section{Dealing with missing data}

Where we identified data as being missing or unclear in the published literature, we contacted trial authors directly. We contacted three authors for additional trial information (Antmen 2013; Badawy 2010; Elalfy 2015) and have received one response stating that the trial data were not available at this time (Badawy 2010). We recorded the number of participants lost to follow-up for each trial. Where possible, we analysed data on an intention-to-treat (ITT) basis, but if insufficient data were available, we also presented a per protocol analyses (Higgins 2011c).

\section{Assessment of heterogeneity}

If the clinical and methodological characteristics of individual trials were sufficiently homogeneous, we combined the data to perform a meta-analysis. We planned to analyse the data from RCTs, NRSIs, CBA and ITS studies separately, but we only included RCTs.

We assessed statistical heterogeneity of treatment effects between trials using a $\mathrm{Chi}^{2}$ test with a significance level at $\mathrm{P}<0.1$. We used the $\mathrm{I}^{2}$ statistic to quantify the degree of potential heterogeneity and classified it as moderate if $\mathrm{I}^{2}$ is greater than $50 \%$, or considerable if $\mathrm{I}^{2}$ is greater than $75 \%$. We used the random-effects model as we anticipated that we would identify at least moderate clinical and methodological heterogeneity within the trials selected for inclusion. If statistical heterogeneity was considerable, we did not report the overall summary statistic. We assessed potential causes of heterogeneity by sensitivity and subgroup analyses (Deeks 2011).

\section{Assessment of reporting biases}

No meta-analysis in this review included at least 10 trials, we therefore could not perform a formal assessment of publication bias (Sterne 2011).

\section{Data synthesis}

If trials were sufficiently homogenous in their design, we conducted a meta-analysis according to the recommendations of Cochrane (Deeks 2011). We used the random-effects model for all analyses as we anticipated that true effects would be related but not the same for included trials. If we could not perform a metaanalysis we commented on the results as a narrative.

For RCTs where meta-analysis was feasible, we used the MantelHaenszel method for dichotomous outcomes and the inverse variance method for continuous outcomes. We did not have outcomes that included data from cluster-RCTs. Where heterogeneity was above $75 \%$, and we identified a cause for the heterogeneity, we explored this with subgroup analyses. If we did not find a cause for the heterogeneity then we did not perform a meta-analysis. If identified, we planned to analyse NRSIs or CBA studies separately. We planned to analyse outcomes with adjusted effect estimates if these were adjusted for the same factors using the inverse variance method as recommended in chapter 13 of the Cochrane Handbook of Systematic Reviews of Interventions (Reeves 2011). For ITS studies, we would have used the effect sizes (if reported in the included studies or obtained (as described earlier)) and pooled them using the generic inverse variance method in Review Manager 5 (RevMan 2014).

\section{Subgroup analysis and investigation of heterogeneity}

We reported results for the different types of disease separately (SCD or thalassaemia). Only one trial included participants with SCD (Vichinsky 2007).

There were insufficient data to perform some of the planned subgroup analyses. We planned to perform subgroup analyses according to Cochrane's recommendations (Deeks 2011) for each of the following criteria, and separately for the different study design types included in the review in order to assess the effect on heterogeneity.

- Age of participant (child (one to 12 years), adolescent (13 to 17 years) adult (18+ years))

- Route of administration of iron chelating agents (oral, intravenous or subcutaneous) 


\section{Sensitivity analysis}

There were insufficient data to perform the planned sensitivity analyses. If adequate data were available, we planned to assess the robustness of our findings by performing the following sensitivity analyses according to Cochrane recommendations where appropriate (Deeks 2011).

- Including only those trials with a 'low' risk of bias (e.g. RCTs with methods assessed as low risk for random sequence generation and concealment of treatment allocation)

- Including only those studies with less than a $20 \%$ dropout rate

- Duration of follow-up (up to and including six months compared to over six months)

\section{Summary of findings table}

We used the GRADE approach to generate a 'Summary of Findings' table as suggested in the Cochrane Handbook for Systematic Reviews of Interventions (Schünemann 2011a). We used the GRADE approach to rate the quality of the evidence as 'high', 'moderate', 'low', or 'very low' using the five GRADE considerations.

- Risk of bias (serious or very serious)

- Inconsistency (serious or very serious)

- Indirectness (serious or very serious)

- Imprecision (serious or very serious)

- Publication bias (likely or very likely)

For NRSIs or CBA or ITS studies, we planned to consider the following factors.

- Dose response (yes or no)

- Size of effect (large or very large)
- Confounding either reduces the demonstrated effect or increases the effect if no effect was observed (yes or no)

In GRADE NRSIs or CBA or ITS studies are rated initially as low quality and upgraded according to GRADE guidelines if appropriate. We planned to present outcomes for these studies in separate tables from outcomes for the results of RCTs.

We reported the following outcomes in each 'Summary of findings' table.

1. Adherence rates (minimum of three months)

2. Serious adverse events (most common time frame used in most studies)

3. All-cause mortality (most common time frame used in most studies)

4. Sustained adherence (six months or more)

5. QoL (most common time frame used in most studies)

\section{R E S U L T S}

\section{Description of studies}

See also Characteristics of included studies; Characteristics of excluded studies; Characteristics of studies awaiting classification; Characteristics of ongoing studies.

\section{Results of the search}

See PRISMA flow diagram (Figure 1). 
Figure I. Study flow diagram.

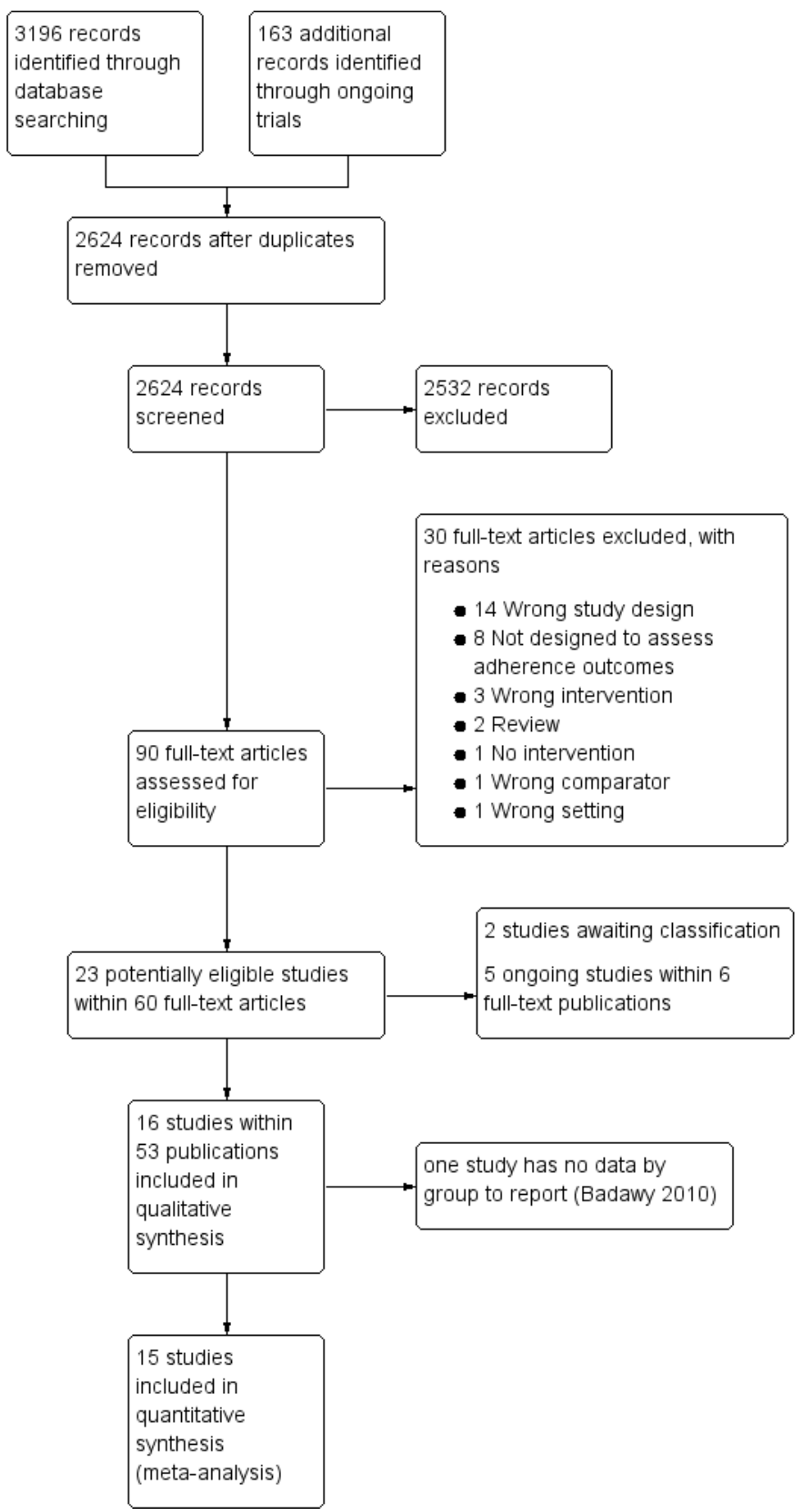


In the searches for this review we identified a total of 3359 potentially relevant references. There were 2624 references after we removed duplicates and three review authors (PF, KM and LE) excluded 2533 references on the basis of abstract and three authors (PF, KM, LE) reviewed 90 full-text articles for relevance.

We excluded 30 studies that were not relevant and identified 16 studies within 53 publications - all were RCTs (Aydinok 2007; Badawy 2010; Bahnasawy 2017; Calvaruso 2015; El Beshlawy 2008; Galanello 2006; Hassan 2016; Maggio 2009; Mourad 2003; Olivieri 1997; Pennell 2006; Pennell 2014; Taher 2017; Tanner 2007; Vichinsky 2007).

We also identified five ongoing RCTs ( IRCT2015101218603N2; EudraCT 2012-000353-31; Madderom 2016; NCT02173951; NCT02435212), and two studies awaiting classification (Antmen 2013; NCT00004982). We did not identify any cluster-randomised trials, NRSIs, CBA or ITS studies that met the inclusion criteria.

\section{Included studies}

Sixteen RCTs including 1525 participants met the pre-defined inclusion criteria (Aydinok 2007; Badawy 2010; Bahnasawy 2017; Calvaruso 2015; Elalfy 2015; El Beshlawy 2008; Galanello 2006; Hassan 2016; Maggio 2009; Mourad 2003; Olivieri 1997; Pennell 2006; Pennell 2014; Taher 2017; Tanner 2007; Vichinsky 2007). Two of the included trials were abstract reports only (Badawy 2010; Olivieri 1997). One abstract did not report outcomes by intervention and therefore is not included in the quantitative reporting of the effects of interventions (Badawy 2010).

\section{Trial design}

There were 15 RCTs of medication interventions (Aydinok 2007; Badawy 2010; Calvaruso 2015; Elalfy 2015; El Beshlawy 2008; Galanello 2006; Hassan 2016; Maggio 2009; Mourad 2003; Olivieri 1997; Pennell 2006; Pennell 2014; Taher 2017; Tanner 2007; Vichinsky 2007); while one was an RCT on medication management (Bahnasawy 2017).

Ten were multicentre trials (Calvaruso 2015; Elalfy 2015; Galanello 2006; Maggio 2009; Olivieri 1997; Pennell 2006; Pennell 2014; Taher 2017; Tanner 2007; Vichinsky 2007) and ranged from two centres in one country (Calvaruso 2015; Elalfy 2015; Olivieri 1997) to 44 centres in multiple countries (Vichinsky 2007). Six were single-centre trials (Aydinok 2007; Bahnasawy 2017; Badawy 2010; El Beshlawy 2008; Hassan 2016, Mourad 2003).

Follow-up ranged from six months in two trials (Bahnasawy 2017; Taher 2017) to five years in two trials (Calvaruso 2015; Maggio 2009). The remainder of the trials were of 12 months duration, except in the Badawy trial, which did not report follow-up time (
Badawy 2010); and the Olivieri trial, which had 24 months followup (Olivieri 1997).

\section{Trial size}

The number of participants enrolled in the trials ranged from 24 (Aydinok 2007) to 213 (Maggio 2009). Sample-size calculations were reported in eight trials (Calvaruso 2015; Elalfy 2015; El Beshlawy 2008; Maggio 2009; Pennell 2006; Pennell 2014; Tanner 2007; Vichinsky 2007).

\section{Setting}

Trials were published between 1997 and 2017. Five were conducted in Egypt (Badawy 2010; Bahnasawy 2017; Elalfy 2015; El Beshlawy 2008, Hassan 2016); five in Italy (Calvaruso 2015; Galanello 2006; Maggio 2009; Pennell 2006; Tanner 2007); and three were international multicentre trials conducted in several countries (Pennell 2014; Taher 2017; Vichinsky 2007). One trial was conducted in each of the following countries: Turkey (Aydinok 2007); Lebanon (Mourad 2003); and Canada (Olivieri 1997).

\section{Participants}

Fourteen trials included only participants with $\beta$-thalassaemia major (Aydinok 2007; Badawy 2010; Bahnasawy 2017; Elalfy 2015; El Beshlawy 2008; Galanello 2006; Hassan 2016; Maggio 2009; Mourad 2003; Olivieri 1997; Pennell 2006; Pennell 2014; Taher 2017; Tanner 2007). One trial included only participants with SCD (Vichinsky 2007); and one trial included only participants with thalassaemia intermedia (Calvaruso 2015).

The mean age ranged from 11 years (El Beshlawy 2008) to 41 years (Calvaruso 2015). Two trials only provided the minimum age of enrolment into the RCT, at least eight years old in the Badawy trial (Badawy 2010); and at least 10 years old in the Olivieri trial (Olivieri 1997).

Participants tended to be equally divided between males and females with the lowest percentage of males at 38\% (Bahnasawy 2017) to a high of $66 \%$ (Elalfy 2015).

\section{Intervention}

In this review we report the Effects of interventions by the various comparisons in the different trials. All trials included medication interventions except for one, which was a medication management intervention by a clinical pharmacist (Bahnasawy 2017).

The comparisons and studies included:

- DFP versus DFO: five trials (Badawy 2010; Calvaruso 2015; El Beshlawy 2008; Olivieri 1997; Pennell 2006); 
- DFX versus DFO: three trials (Hassan 2016; Pennell 2014; Vichinsky 2007);

- DFX (film-coated tablet (FCT) versus DFX (dispersible tablet (DT)): one trial (Taher 2017);

- DFP and DFO combined versus DFP alone: four trials (Aydinok 2007; Badawy 2010; El Beshlawy 2008; Maggio 2009);

- DFP and DFO combined versus DFO alone: five trials (Badawy 2010; El Beshlawy 2008; Galanello 2006; Mourad 2003; Tanner 2007);

- DFP and DFO combined versus DFP and DFX

combined: one trial (Elalfy 2015);

- Medication management versus standard care: one trial (Bahnasawy 2017).

\section{Outcomes}

Outcomes varied across trials depending on the objectives. All trials measured adherence, although this was usually as a secondary, rather than a primary outcome. Reduction in serum ferritin or LIC were the primary outcomes in most trials; however, in three trials the primary outcome was myocardial T2* MRI results (Pennell 2006; Pennell 2014; Tanner 2007) and in one trial was overall safety (Taher 2017). Safety (including both SAEs and AEs) was included as a secondary outcome in all trials. QoL was reported in three trials (Aydinok 2007; Bahnasawy 2017; Elalfy 2015).

\section{Source}

Four trials identified non-profit organisations as their source of support, including universities, foundations and societies (Badawy 2010; Calvaruso 2015; Elalfy 2015; Maggio 2009).

Five trials identified industry sponsorships (Galanello 2006; Pennell 2006; Pennell 2014; Taher 2017; Vichinsky 2007). Six trials did not state their source of funding (Aydinok 2007; Bahnasawy
2017; El Beshlawy 2008; Hassan 2016; Mourad 2003; Olivieri 1997); but of these, three may have had industry funding. In one trial, drugs were supplied by the manufacturer (Aydinok 2007); one trial was halted by the manufacturer (Olivieri 1997); and one trial included industry employees as authors (El Beshlawy 2008). One trial had a mix of non-profit and industry funding (Tanner 2007).

\section{Excluded studies}

We excluded 30 trials:

- in 14 trials the trial design did not meet the inclusion criteria (Abu 2015; Al Kloub 2014; Al Kloub 2014a; Al Refaie 1995; Alvarez 2009; Kidson Gerber 2008; Kolnagou 2008; Leonard 2014; NCT02133560; NCT02466555; Pakbaz 2004; Pakbaz 2005; Porter 2009; Porter 2012);

- eight trials were not designed to assess adherence (Berkovitch 1995; Chakrabarti 2013; NCT01709032; NCT01825512; Vichinsky 2005; Vichinsky 2008; Waheed 2014; Yarali 2006);

- three trials assessed the wrong intervention (Armstrong 2011, Belgrave 1989; Gomber 2004);

- one trial had no interventions (Bala 2014);

- one trial had a wrong comparator (Mazzone 2009);

- one trial was in the wrong setting (Daar 2010);

- two were reviews (Loiselle 2016; Walsh 2014).

\section{Risk of bias in included studies}

Refer to the figures section of the review for visual representations of the assessments of risk of bias across all trials and for each item in the included trials (Figure 2; Figure 3). See the risk of bias section in the Characteristics of included studies section for further information about the bias identified within individual trials. 
Figure 2. Risk of bias graph: review authors' judgements about each risk of bias item presented as percentages across all included studies.

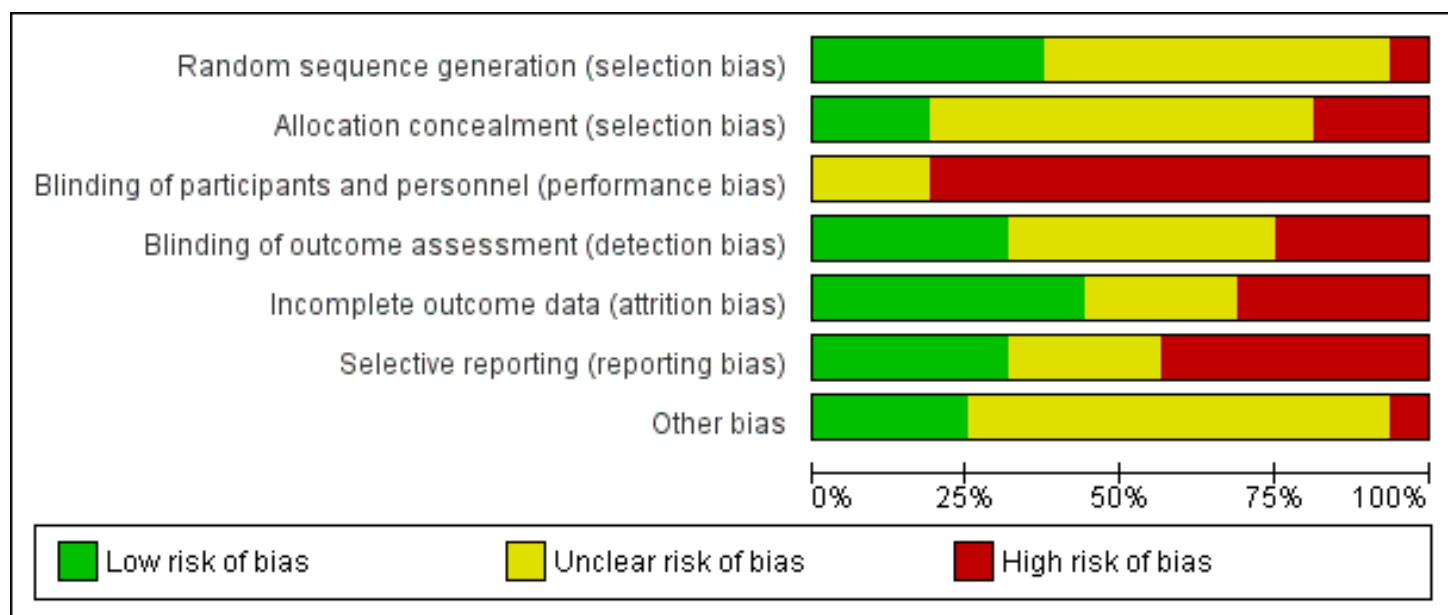


Figure 3. Risk of bias summary: review authors' judgements about each risk of bias item for each included study.

\begin{tabular}{|c|c|c|c|c|c|c|c|}
\hline & 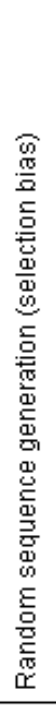 & 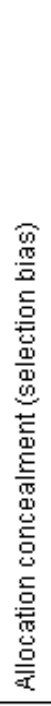 & 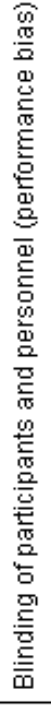 & 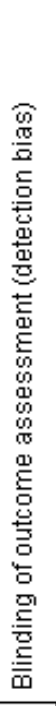 & 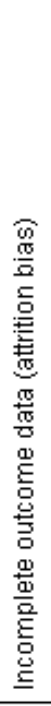 & 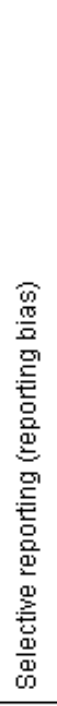 & 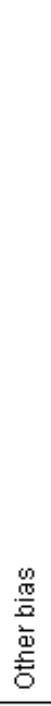 \\
\hline Aydinok 2007 & + & - & & $?$ & . & + & $?$ \\
\hline Badaw 2010 & $?$ & $?$ & & $?$ & - & & $?$ \\
\hline Bahnasawy 2017 & $?$ & $?$ & & $\partial$ & . & (- & $?$ \\
\hline Calvaruso 2015 & + & + & & + & + & + & $?$ \\
\hline Elalfy 2015 & + & + & & + & + & $?$ & $?$ \\
\hline El Beshlawy 2008 & $?$ & $?$ & & $?$ & 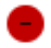 & & $?$ \\
\hline Galanello 2006 & $?$ & $?$ & $?$ & $?$ & 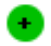 & $?$ & + \\
\hline Hassan 2016 & $?$ & $?$ & & - & $\oplus$ & 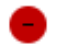 & $?$ \\
\hline Maggio 2009 & + & + & 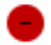 & + & $?$ & + & $?$ \\
\hline Mourad 2003 & $?$ & $?$ & $?$ & $?$ & + & 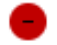 & + \\
\hline Olivieri 1997 & A & $?$ & & $?$ & & + & $?$ \\
\hline Pennell 2006 & $?$ & $?$ & & + & $\oplus$ & 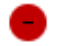 & \\
\hline Pennell 2014 & + & $?$ & & + & $?$ & $?$ & + \\
\hline Taher 2017 & $?$ & & & & $?$ & & $?$ \\
\hline Tanner 2007 & $?$ & ( & $?$ & $?$ & $?$ & + & $\odot$ \\
\hline Vichinsky 2007 & + & $?$ & & & $\oplus$ & $?$ & $?$ \\
\hline
\end{tabular}


Allocation

\section{Random sequence generation}

We considered six trials to be at a low risk of bias for random sequence generation as randomisation was clearly described and done centrally, in permuted blocks, or computer-generated (Aydinok 2007; Calvaruso 2015; Elalfy 2015; Maggio 2009; Pennell 2014; Vichinsky 2007).

We considered nine trials to be at an unclear risk of bias. Although one trial used permuted blocks there were several imbalances in baseline characteristics between groups (Hassan 2016). We judged the remaining eight trials to have an unclear risk of bias as there was no description of randomisation and the report only stated that participants were randomised (Badawy 2010; Bahnasawy 2017; El Beshlawy 2008; Galanello 2006; Mourad 2003; Pennell 2006; Taher 2017; Tanner 2007).

We considered one trial to be at a high risk of bias as participants were "assigned" to treatment groups by a research pharmacist and there was no description of how it was done (Olivieri 1997).

\section{Allocation concealment (selection bias)}

We considered three trials to be at low risk for selection bias as participants were allocated by telephone contact from a co-ordinating centre (Calvaruso 2015; Elalfy 2015; Maggio 2009).

We considered 10 trials to be at an unclear risk as there was no description of how allocation was concealed (Badawy 2010; Bahnasawy 2017; El Beshlawy 2008; Galanello 2006; Hassan 2016; Mourad 2003; Olivieri 1997; Pennell 2006: Pennell 2014; Vichinsky 2007).

We considered three trials to be at a high risk for selection bias as there was no allocation concealment (Aydinok 2007; Taher 2017; Tanner 2007).

\section{Blinding}

\section{Blinding of participants and personnel (performance bias)}

We considered three trials to be at an unclear risk for performance bias as there was no description of blinding (Galanello 2006; Mourad 2003; Tanner 2007).

We considered 13 trials to be at a high risk for performance bias. Trials were either open label, did not mention blinding, or blinding was difficult due to type of treatment: a subcutaneous injection compared to an oral intervention or combination of both (Aydinok 2007; Badawy 2010; Bahnasawy 2017; El Beshlawy 2008; Calvaruso 2015; Elalfy 2015; Hassan 2016; Maggio 2009;
Olivieri 1997; Pennell 2006; Pennell 2014; Taher 2017; Vichinsky 2007).

\section{Blinding of outcome assessment (detection bias)}

We considered five trials to be at a low risk of detection bias for all outcomes as data management and analysis were carried out by assessors who were blinded to interventions (Calvaruso 2015; Elalfy 2015; Maggio 2009; Pennell 2006; Pennell 2014).

We considered seven trials to be at an unclear risk of detection bias for all outcomes except mortality as there was no mention of blinding (Aydinok 2007; Badawy 2010; El Beshlawy 2008; Galanello 2006; Mourad 2003; Olivieri 1997; Tanner 2007).

We considered four trials to be at a high risk of detection bias as there was no description of blinding of outcome assessment and it appears that investigators who were not blinded were also involved in outcome assessment (Bahnasawy 2017; Hassan 2016; Taher 2017; Vichinsky 2007).

\section{Incomplete outcome data}

We considered seven trials to be at a low risk for attrition bias as all outcomes were reported and either no participants or few participants were lost to follow-up and flow of participants was reported (Calvaruso 2015; Elalfy 2015; Galanello 2006; Hassan 2016; Mourad 2003; Pennell 2006; Vichinsky 2007).

We considered four trials to be at an unclear risk of attrition bias as there was no indication of the number of participants included in the different outcome analyses; there was substantial attrition towards the end of the trial, a per protocol analysis was conducted for some outcomes; or there was high attrition or vague reporting with no specific results (Maggio 2009; Pennell 2014; Taher 2017; Tanner 2007).

We considered the rest of the trials to be at a high risk for attrition bias as there was no data on the flow and number of participants completing the trial; no participant numbers on adverse events or compliance; no comparative data reported; per protocol analysis only; or large attrition bias in outcome analysis (Aydinok 2007; Badawy 2010; Bahnasawy 2017; El Beshlawy 2008; Olivieri 1997).

\section{Selective reporting}

We considered five trials to be at a low risk of reporting bias as all identified outcomes were reported (Aydinok 2007; Calvaruso 2015; Maggio 2009; Olivieri 1997; Tanner 2007).

We considered four trials to be at an unclear risk of reporting bias because of either: minimal reporting of participant satisfaction and compliance; or no report of compliance with DFP; or unclear and 
selective reporting of adverse events (Elalfy 2015; Galanello 2006; Pennell 2014; Vichinsky 2007).

We considered seven trials to be at a high risk of reporting bias due to: the incomplete reporting of adverse events or a lack of reporting of adverse events by treatment groups; or a lack of detailed or incomplete reporting of compliance and serum ferritin and LIC; or non-reporting of some pre-specified outcomes (Badawy 2010, Bahnasawy 2017; El Beshlawy 2008; Hassan 2016, Mourad 2003; Pennell 2006; Taher 2017).

\section{Other potential sources of bias}

We considered four trials to be at a low risk as no other potential sources of bias were identified (Galanello 2006; Mourad 2003; Pennell 2014; Tanner 2007).

We considered 11 trials to be at an unclear risk of other bias for various reasons including: baseline imbalances; abstract reports with insufficient details; no comparative numbers in control group; incomplete reporting of AEs; dose amendments after the start of the trial (Aydinok 2007; Badawy 2010; Bahnasawy 2017; Calvaruso 2015; Elalfy 2015; El Beshlawy 2008; Hassan 2016; Maggio 2009; Olivieri 1997; Taher 2017; Vichinsky 2007).

We considered one trial to be at a high risk of other sources of bias due to a serious imbalance in baseline characteristics of participants, particularly serum ferritin levels (Pennell 2006).

\section{Effects of interventions}

See: Summary of findings for the main comparison DFP compared to DFO for improving adherence to iron chelation therapy in people with sickle cell disease or thalassaemia; Summary of findings 2 DFX compared to DFO for improving adherence to iron chelation therapy in people with sickle cell disease or thalassaemia; Summary of findings 3 DFX filmcoated tablet compared to DFX dispersible tablet for improving adherence to iron chelation therapy in people with sickle cell disease or thalassaemia; Summary of findings 4 DFP and DFO compared to DFP for improving adherence to iron chelation therapy in people with sickle cell disease or thalassaemia; Summary of findings $5 \mathrm{DFP}$ and DFO compared to DFO for improving adherence to iron chelation therapy in people with sickle cell disease or thalassaemia; Summary of findings 6 DFP and DFO compared to DFP and DFX for improving adherence to iron chelation therapy in people with sickle cell disease or thalassaemia; Summary of findings 7 Medication management compared to standard care for improving adherence to iron chelation therapy in people with sickle cell disease or thalassaemia Results are presented for each of the main comparisons.

The main focus of our review is on compliance and effects of compliance (or non-compliance) on participant outcomes. For more detailed estimates of effectiveness of different iron chelators please refer to another Cochrane Review (Fisher 2013).
One abstract of a trial that included three review comparisons (DFP versus DFO; combination DFP and DFO versus DFP; combination DFP and DFO versus DFO) did not report any outcomes by intervention group and did not include counts of events (i.e. adverse events); therefore we did not include this trial in the quantitative analysis (Badawy 2010). Thus we have included 15 trials within the quantitative analysis.

See Table 1 and also the outcomes section in the Characteristics of included studies section for summary information on results and how adherence was measured in the individual trials. Adherence rates were mostly measured by pill or vial count (either automated or manual).

The quality of the evidence has been graded for those outcomes included in the summary of findings table. For the definitions of these gradings, please refer to the summary of findings tables (Summary of findings for the main comparison; Summary of findings 2; Summary of findings 3; Summary of findings 4; Summary of findings 5; Summary of findings 6; Summary of findings 7).

\section{DFP (deferiprone) alone versus DFO (deferoxamine) alone}

Four trials of thalassaemia met the inclusion criteria for this comparison (Calvaruso 2015; El Beshlawy 2008; Olivieri 1997; Pennell 2006). See Summary of findings for the main comparison.

\section{Primary outcomes}

\section{Adherence to iron chelation therapy rates}

We are uncertain whether oral DFP increases adherence to iron chelation therapy more than subcutaneous DFO (very low-quality evidence). Results could not be combined due to both a lack of data to report as well as considerable heterogeneity between comparisons $\left(\mathrm{I}^{2}=99 \%\right.$ ) (Analysis 1.1$)$. We identified the age of participants and differences in the medication regimens as possible explanations for heterogeneity. We provide a narrative review of the data on compliance below.

- Calvaruso 2015: compliance with DFP: 85\% (47 participants) versus compliance with DFO: $76 \%$ (41 participants).

- El Beshlawy 2008: "four patients, all treated with DFObased regimen, were excluded from the study due to lack of compliance. Compliance was otherwise excellent during the entire study period".

- Olivieri 1997: compliance with DFP: $94.9 \% \pm 1.1 \%$ (19 participants) versus compliance with DFO: $71.6 \% \pm 3.9 \%$ (18 participants).

- Pennell 2006: compliance with DFP: $94 \% \pm 5.3 \%$ (29 participants) versus compliance with DFO: $93 \% \pm 9.7 \%(32$ participants). 


\section{Serious adverse events (SAEs)}

Two trials reported this outcome (Calvaruso 2015; Pennell 2006). One trial reported on the risk of developing agranulocytosis: we are uncertain if switching to oral DFP increases the risk of agranulocytosis compared to subcutaneous DFO, RR 7.88 (95\% CI 0.18 to 352.39 ) (one trial; 88 participants; very low-quality evidence) (Calvaruso 2015) (Analysis 1.2). No SAEs occurred in the second trial (Pennell 2006).

\section{All-cause mortality}

Two trials reported this outcome (Calvaruso 2015; Pennell 2006). Oral DFP may have little or no difference on mortality compared to subcutaneous DFO, RR 0.44 (95\% CI 0.12 to 1.63) (88 participants; one trial; low-quality evidence) (Calvaruso 2015) (Analysis 1.3). No deaths occurred in the second trial (Pennell 2006).

\section{Secondary outcomes}

\section{Sustained adherence to therapy (measured for a minimum of six months)}

All trials reported more than six months follow-up, sustained adherence is reported in the primary outcome (adherence to iron chelation therapy rates), as only end-of-trial adherence numbers were provided.

\section{Health-related quality of life (QoL)}

No trials measured QoL.

\section{Iron overload}

One trial reported the proportion of participants with iron overload (Calvaruso 2015). We are uncertain if DFP reduces iron overload compared to DFO: iron levels greater or equal to $800(\mu \mathrm{g} / \mathrm{L})$, RR 1.31 (95\% CI 0.49 to 3.48) (one trial; 38 participants; very low quality evidence) (Analysis 1.4).

\section{Organ damage}

One trial reported the proportion of participants with organ damage (Calvaruso 2015). We are uncertain if DFP increases the risk of liver damage compared to DFO, RR 4.36 (95\% CI 0.53 to 35.82) (one trial; 88 participants; very low-quality evidence) (Analysis 1.5).

\section{Other adverse events (AEs) related to iron chelation}

Three trials reported this outcome (Calvaruso 2015; El Beshlawy 2008; Pennell 2006). In people with thalassaemia taking DFP, we are uncertain if there is a difference in the risk of AEs compared to people taking DFO (Analysis 1.6).

- Risk of leukopenia: RR 3.94 (99\% CI 0.44 to 35.50) (three trials; 192 participants; very low-quality evidence) (Calvaruso 2015; El Beshlawy 2008; Pennell 2006).

- Risk of pain or swelling in joints: RR 3.38 (99\% CI 0.54 to 21.31) (three trials; 192 participants; very low-quality evidence) (Calvaruso 2015; El Beshlawy 2008; Pennell 2006).

- Risk of nausea or vomiting: RR 13.68 (99\% CI 0.99 to 188.88) (two trials; 132 participants; very low-quality evidence) (Calvaruso 2015; El Beshlawy 2008).

- Risk of increased liver transaminase: RR 1.10 (99\% CI 0.03 to 38.47) (one trial; 44 participants; very low-quality evidence) (El Beshlawy 2008).

- Local reactions at infusions site: RR 0.17 (99\% CI 0.00 to 9.12) (one trial; 88 participants; very low-quality evidence) (Calvaruso 2015).

In all trials we downgraded the quality of evidence by one for risk of bias (due to high or unclear risk of bias in several domains) and in one trial we downgraded by two due to imprecision, the effect estimates have wide CIs (Calvaruso 2015).

\section{DFX (deferasirox) alone versus DFO (deferoxamine) alone}

Three trials met the inclusion criteria for this comparison; two in thalassaemia (Hassan 2016; Pennell 2014); and one in SCD (Vichinsky 2007). See Summary of findings 2.

\section{Primary outcomes}

\section{Adherence to iron chelation therapy rates}

All three trials reported on this outcome. Only one trial reported data in a format that could be incorporated into the analysis (Pennell 2014). We are uncertain if DFX increases the rate of adherence compared to people taking DFO, MD -1.40 (95\% CI -3.66 to 0.86 ) (one trial; 197 participants; very-low quality evidence) (Analysis 2.1).

Regarding the remaining two trials:

- Hassan 2016 stated that "throughout the study, all patients were compliant with the prescribed doses, and no discontinuation of drugs or drop-out of follow-up occurred."

- Vichinsky 2007 reported that "the ratios of the administered to intended doses of therapy were high (1.16 for deferasirox and 0.97 for deferoxamine), indicating high adherence to the prescribed treatment regimens." 


\section{Serious adverse events (SAEs)}

All three trials reported the effect on disease-related SAEs (Hassan 2016; Pennell 2014; Vichinsky 2007); two in thalassaemia (Hassan 2016; Pennell 2014), and one in SCD (Vichinsky 2007).

We are uncertain whether DFX decreases risk of disease-related SAEs in thalassaemia compared to DFO, RR 0.95 (95\% CI 0.41 to 2.17) (two trials; 247 participants; very low-quality evidence) (Analysis 2.2).

We are uncertain whether DFX decreases the risk of SCD-related pain crisis, RR 1.05 (95\% CI 0.68 to 1.62 ) (one trial; 195 participants; very low-quality evidence); or other SCD-related SAEs compared to DFO, RR 1.08 (95\% CI 0.77 to 1.51 ) (one trial; 195 participants; very low-quality evidence) (Analysis 2.2).

\section{All-cause mortality}

Two trials report mortality (Hassan 2016; Pennell 2014). We are uncertain whether DFX decreases the risk of mortality in people with thalassaemia compared to DFO, RR 0.96 (95\% CI 0.06 to 15.06) (two trials; 240 participants; very low-quality evidence) (Analysis 2.3).

\section{Secondary outcomes}

\section{Sustained adherence to therapy (measured for a minimum of six months)}

All trials reported more than six months follow-up, sustained adherence is reported in the primary outcome (adherence to iron chelation therapy rates), as only end-of-trial adherence numbers were provided.

\section{Health-related quality of life (QoL)}

No trials measured quality of life.

\section{Iron overload}

In people with thalassaemia, we are uncertain whether DFX reduces the proportion of participants with serum ferritin of 1500 $(\mu \mathrm{g} / \mathrm{l})$ or higher, RR 1.18 (95\% CI 0.63 to 2.20 ) (one trial; 60 participants; very low-quality evidence) (Hassan 2016) (Analysis 2.4). Furthermore, we are uncertain whether DFX reduces the proportion of participants with severe LIC $(15 \mathrm{mg} \mathrm{Fe} / \mathrm{g} \mathrm{dw}$ or higher), RR 1.00 (95\% CI 0.83 to 1.20 ); or myocardial T2* $<10$ ms, RR 1.10 (95\% CI 0.72 to 1.70 ) (one trial; 172 participants; very low-quality evidence)* (Pennell 2014) (Analysis 2.4).

*LIC and myocardial T2*analyses from Pennell 2014 were based on the per protocol population.

In people with SCD, Vichinsky reported LIC mean changes from baseline and no data on proportion of participants with end-oftrial iron overload (Vichinsky 2007).

\section{Organ damage}

No trial reported any other organ damage.

\section{Other adverse events (AEs) related to iron chelation}

In people with thalassaemia taking DFX, we are uncertain if there is a difference in the risk of iron chelation therapy-related AEs compared to people taking DFO (Analysis 2.5).

- Risk of total iron chelation therapy-related AE: RR 1.15 (95\% CI 0.76 to 1.73 ); (one trial; 187 participants; very lowquality evidence) (Pennell 2014).

- Risk of gastrointestinal upset: RR 3.00 (95\% CI 0.66 to 13.69); (one trial; 60 participants; very low-quality evidence) (Hassan 2016).

- Risk of rash: RR 3.05 (95\% CI 0.98 to 9.47); (two trials; 247 participants; very low-quality evidence) (Hassan 2016; Pennell 2014).

- Risk of increased blood creatinine: RR 3.79 (95\% CI 0.83 to 17.38 ); (one trial;187 participants; very low-quality evidence) (Pennell 2014).

- Risk of proteinuria: RR 2.21 (95\% CI 0.59 to 8.29); (one trial; 187 participants; very low-quality evidence) (Pennell 2014).

- Risk of increased ALT: RR 5.69 (95\% CI 0.70 to 46.33); (one trial; 187 participants; very low-quality evidence); (Pennell 2014).

- Risk of increased AST: RR 5.69 (95\% CI 0.70 to 46.33); (one trial; 187 participants; very low-quality evidence); (Pennell 2014).

- Risk of diarrhoea: RR 5.69 (95\% CI 0.70 to 46.33); (one trial; 187 participant; very low-quality evidence); (Pennell 2014).

- Risk of vomiting: RR 6.64 (95\% CI 0.35 to 126.78); (one trial; 187 participants; very low-quality evidence); (Pennell 2014).

In people with thalassaemia, we are uncertain whether DFX reduces the incidence of total AEs as compared to DFO, RR 0.89 (95\% CI 0.75 to 1.07 ) (one trial; 187 participants; very low-quality evidence) (Pennell 2014) (Analysis 2.6). We downgraded the quality of evidence either by two due to high or uncertain risk of bias in several domains, or by one due to imprecision as CIs are wide and only one trial with data in comparison, or both.

In people with SCD, DFX compared to DFO, may increase slightly the risk of abdominal pain, RR 1.91 ( $99 \%$ CI 0.80 to 4.58 ); the risk of diarrhoea, RR 4.14 (99\% CI 0.90 to 18.92$)$; and the risk of nausea or vomiting, RR 1.63 (99\% CI 0.90 to 2.94) (one trial; 195 participants; low-quality evidence) (Vichinsky 2007) (Analysis 2.7). We are uncertain if DFX compared to DFO increases the risk of an increase in ALT, RR 5.29 (99\% CI 0.12 to 232.98) or the risk of pain or swelling in joints, RR 1.06 (99\% CI 0.41 to 2.76) (one trial; 195 participants; very low-quality evidence) (Vichinsky 2007) (Analysis 2.7). We downgraded the quality of evidence either by two due to high or uncertain risk of bias in 
several domains, or by one due to imprecision as CIs are wide and only one trial with data in comparison, or both.

\section{DFX film-coated Tablet (FCT) versus DFX (deferasirox) dispersible tablet (DT)}

One trial in thalassaemia met the inclusion criteria for this comparison (Taher 2017). See Summary of findings 3.

\section{Primary outcomes}

\section{Adherence to iron chelation therapy rates}

DFX FCT may have little or no difference on adherence as compared to DFX DT, RR 1.10 (95\% CI 0.99 to 1.22 ) (one trial; 173 participants; low-quality evidence) (Analysis 3.1).

\section{Serious adverse events (SAEs)}

We are uncertain if DFX FCT increases SAEs as compared to DFX DT, RR 1.22 (95\% CI 0.62 to 2.37) (one trial; 173 participants; very low-quality evidence) (Analysis 3.2).

\section{All-cause mortality}

We are uncertain if DFX FCT increases all-cause mortality as compared to DFX DT, RR 2.97 (95\% CI 0.12 to 71.81) (one trial; 173 participants; very low-quality evidence) (Analysis 3.3).

\section{Secondary outcomes}

\section{Sustained adherence to therapy}

This trial reported more than six months follow-up, sustained adherence is reported in the primary outcome (adherence to iron chelation therapy rates), as only end of trial adherence numbers were provided.

\section{Health-related quality of life (QoL)}

This outcome was not measured with a validated instrument.

\section{Iron overload}

The trial did not report the proportion of participants with iron overload at the end of the trial.

\section{Organ damage}

We are uncertain if DFX FCT increases the incidence of renal events as compared to DFX DT, RR 1.25 ( $95 \%$ CI 0.83 to 1.91 ) (one trial; 173 participants; very low-quality evidence) (Analysis 3.4).

\section{Other adverse events (AEs) related to iron chelation}

DFX FCT, compared to DFX DT, may improve slightly the incidence of all chelation-related AEs, RR 0.75 (99\% CI 0.52 to 1.08); and the incidence of vomiting, RR 0.28 (99\% CI 0.07 to 1.15) (one trial; 173 participants; low-quality evidence) (Analysis 3.5).

We are uncertain if DFX FCT, compared to DFX DT, improves: the risk of diarrhoea, RR 0.70 (99\% CI 0.29 to 1.70); the incidence of abdominal pain, RR 0.49 ( $99 \%$ CI 0.16 to 1.52 ); the incidence of nausea, RR 0.72 (99\% CI 0.23 to 2.23 ) or increases urine protein/urine creatinine ratio, RR 1.65 (99\% CI 0.60 to 4.54 ) (one trial; 173 participants; very low-quality evidence) (Analysis 3.5).

We downgraded the quality of evidence by either two for risk of bias due to high or unclear risk of bias in all domains or by one for imprecision due to wide confidence intervals, or both.

\section{DFP (deferiprone) and DFO (deferoxamine) combination therapy versus DFP (deferiprone) alone}

Three trials in thalassaemia met the inclusion criteria for this comparison (Aydinok 2007; El Beshlawy 2008; Maggio 2009). See Summary of findings 4 .

\section{Primary outcomes}

\section{Adherence to iron chelation therapy rates}

All trials reported on this outcome. We are uncertain if DFP and DFO increases adherence compared to DFP alone (very low-quality evidence).

- Aydinok 2007: "Compliance was generally excellent during the entire study period. There was only one patient in the DFP treatment arm who missed more than one chelation dose per week because of problems with swallowing."

- El Beshlawy 2008: "four patients, all treated with DFObased regimen, were excluded from the study due to lack of compliance. Compliance was otherwise excellent during the entire study period."

- Maggio 2009: "In the sequential DFP-DFO group, compliance was $92.7 \%$ (SD $\pm 15.2 \%$; range $37-100 \%$ ) with DFP treatment and $70.6 \%(S D \pm 24.1 \%$; range $25-100 \%)$ with DFO treatment (105 participants). Compliance with DFP was $93.6 \%(\mathrm{SD} \pm 9.7 \%$; range $56-100 \%)$ in the DFP-alone patients (108 participants).” 


\section{Serious adverse events (SAEs)}

Only one trial reported this outcome (Maggio 2009). In people with thalassaemia, combination therapy with DFP and DFO may have little or no difference on the incidence of SAEs as compared to DFP alone, RR 0.15 (95\% CI 0.01 to 2.81) (one trial; 213 participants; low-quality evidence) (Maggio 2009) (Analysis 4.1).

\section{All-cause mortality}

Two trials reported on this outcome (Aydinok 2007; Maggio 2009). We are uncertain if combination therapy with DFP and DFO decreases mortality as compared to DFP alone, RR 0.77 (95\% CI 0.18 to 3.35) (two trials; 237 participants; very lowquality evidence) (Analysis 4.2).

\section{Secondary outcomes}

\section{Sustained adherence to therapy}

Sustained adherence is reported under the primary outcome (adherence to iron chelation rates), as all trials are longer than six months and end-of-trial adherence is reported.

\section{Health-related quality of life (QoL)}

One trial assessed QoL, but did not use a validated questionnaire (Aydinok 2007).

\section{Iron overload}

No trial reported the proportion of participants with iron overload.

\section{Organ damage}

No trial reported the proportion of participants with organ damage.

\section{Other adverse events (AEs) related to iron chelation}

All three trials reported AEs. We are uncertain if combination DFP and DFO reduces the incidence of adverse events compared to DFP alone in people with thalassaemia (Analysis 4.3).

- Risk of leukopenia, neutropenia or agranulocytosis (or a combination of): RR 1.15 (99\% CI 0.50 to 2.62) (three trials; 280 participants; very low-quality evidence) (Aydinok 2007; El Beshlawy 2008; Maggio 2009).

- Risk of pain or swelling in joints: RR 0.76 (99\% CI 0.31 to 1.91) (two trials; 256 participants; very low-quality evidence) (El Beshlawy 2008; Maggio 2009).

- Risk of increased liver transaminase: RR 1.02 (99\% CI 0.52 to 1.98 ) (two trials; 256 participants; very low-quality evidence) (El Beshlawy 2008; Maggio 2009).
- Risk of nausea or vomiting: RR 0.55 (99\% CI 0.13 to 2.23) (one trial; 43 participants; very low-quality evidence) (El Beshlawy 2008).

One trial reported on this outcome (Maggio 2009). Combination therapy with DFP and DFO may have little or no difference on the risk of gastrointestinal disorders as compare to DFP alone: RR 0.45 (95\% CI 0.15 to 1.37 ) (one trial; 213 participants; lowquality evidence) (Analysis 4.3).

We downgraded the quality of evidence by either two for risk of bias due to high or unclear risk of bias in several domains in all trials, or by one due to imprecision, the effect estimates have wide confidence intervals, or both.

\section{DFP (deferiprone) and DFO (deferoxamine) combination therapy versus DFO (deferoxamine) alone}

Four trials in thalassaemia met the inclusion criteria for this comparison (El Beshlawy 2008; Galanello 2006; Mourad 2003; Tanner 2007). See Summary of findings 5.

\section{Primary outcomes}

\section{Adherence to iron chelation therapy rates}

In people with thalassaemia, combined therapy with DFP and DFO versus DFO alone, may have little or no difference in adherence rates (low-quality evidence). We could not combine any data for an effect estimate.

- El Beshlawy 2008: "four patients, all treated with DFObased regimen, were excluded from the study due to lack of compliance. Compliance was otherwise excellent during the entire study period".

- Galanello 2006: DFP/DFO: DFO: $96.1 \pm 5.0(29$ participants); DFP compliance was not reported; DFO: $95.7 \pm$ 5.7 (30 participants).

- Mourad 2003: "In patients receiving the combined therapy, compliance was excellent (arbitrarily defined as taking $>90 \%$ of the recommended doses) in 10 patients and good (75\% to $90 \%$ of recommended doses) in one patient, as assessed by the patient's history, parental evidence and usage of tablets provided in just sufficient quantities between check-up visits. In patients receiving DFX alone, compliance was considered to be excellent in 11 patients and good in three patients, as assessed mainly by counting the vials given to, and returned by, the patients".

- Tanner 2007: "Compliance with deferoxamine was similar in both groups (combined $91.4 \pm 2.7 \%$ versus deferoxamine $92.6 \pm 2.7 \% ; \mathrm{P}=0.7$ ). Compliance with deferiprone was less than compliance with placebo $(82.4 \pm 18.1 \%$ versus $89.8 \pm$ $7.2 \% ; \mathrm{P}=0.04) \%$. 


\section{Serious adverse events (SAEs)}

Three trials reported SAEs (Galanello 2006; Mourad 2003; Tanner 2007). In people with thalassaemia, combined therapy with DFP and DFO versus DFO alone, may have little or no difference in SAEs (low-quality evidence). No SAEs occurred in the three trials.

\section{All-cause mortality}

Only one trial reported on this outcome and no deaths occurred (Tanner 2007). Combined therapy with DFP and DFO versus DFO alone, may have little or no difference in morality (one trial; 65 participants; low-quality evidence).

\section{Secondary outcome}

\section{Sustained adherence to therapy}

All trials reported more than six months follow-up, sustained adherence is reported in the primary outcome (adherence to iron chelation therapy rates), as only end-of-trial adherence numbers were provided.

\section{Health-related quality of life (QoL)}

No trials measured QoL.

\section{Iron overload}

No trials reported the proportion of participants with iron overload.

\section{Organ damage}

No trials reported the proportion of participants with organ damage.

\section{Other adverse events (AEs) related to iron chelation}

All four trials reported some AEs. We are uncertain if DFP combined with DFO reduces other chelation-related AEs compared to DFO alone in people with thalassaemia (Analysis 5.1).

- Risk of leukopenia, neutropenia or agranulocytosis (or a combination of): RR 1.18 (99\% CI 0.09 to 15.37) (three trials; 169 participants; very low-quality evidence) (El Beshlawy 2008; Galanello 2006; Tanner 2007).

- Risk of pain or swelling in joints: RR 2.39 (99\% CI 0.18 to 32.31) (three trials; 135 participants; very low-quality evidence $\left.I^{2}=66 \%\right)($ El Beshlawy 2008; Mourad 2003; Tanner 2007).

- Risk of increased liver transaminase: RR 3.46 (99\% CI 0.45 to 26.62) (two trials; 104 participants; very low-quality evidence) (El Beshlawy 2008; Galanello 2006).
- Risk of nausea or vomiting: RR 3.81 (99\% CI 0.84 to 17.36) (four trials; 194 participants; very low-quality evidence) (El Beshlawy 2008; Galanello 2006; Mourad 2003; Tanner 2007).

- Risk of local reactions at infusion site: RR 0.18 (99\% CI 0.01 to 3.56 ) (two trials; 90 participants; very low-quality evidence) (Mourad 2003; Tanner 2007).

We downgraded the quality of evidence by two for risk of bias due to high or unclear risk of bias in several domains in all trials and by one due to imprecision, the effect estimates have wide CIs.

\section{Combination DFP (deferiprone) and DFO (deferoxamine) versus combination DFP (deferiprone) and DFX (deferasirox)}

One trial in thalassaemia met the inclusion criteria for this comparison (Elalfy 2015). See Summary of findings 6.

\section{Primary outcomes}

\section{Adherence to iron chelation therapy rates}

In children with thalassaemia, combination therapy with DFP and DFX may improve adherence to iron chelation therapy compared to combination therapy with DFP and DFO, RR 0.84 (95\% CI 0.72 to 0.99 ) (one trial; 96 participants; low-quality evidence) (Analysis 6.1).

\section{Serious adverse events (SAEs)}

In children with thalassaemia, we are uncertain if combination therapy with DFP and DFX decreases the incidence of SAEs compared to combination therapy with DFP and DFO, RR 1.00 (95\% CI 0.06 to 15.53 ) (one trial; 96 participants; very low-quality evidence) (Analysis 6.2).

\section{All-cause mortality}

In children with thalassaemia, combination therapy with DFP and DFX may make little or no difference in mortality compared to combination therapy with DFP and DFO. There were no deaths in the trial (one trial; 96 participants; low-quality evidence).

\section{Secondary outcomes}

\section{Sustained adherence to therapy}

The trial reported more than six months follow-up, sustained adherence is reported in the primary outcome (adherence to iron chelation therapy rates), as only end-of-trial adherence numbers were provided. 


\section{Health-related quality of life (QoL)}

In children with thalassaemia we are unclear if combination therapy with DFP and DFX improves QoL compared to combination therapy with DFP and DFO (very low-quality evidence). Authors state that "significant improvement in quality of life was observed in both groups at study end compared to baseline"; no comparative data were provided.

\section{Iron overload}

Proportion of participants with iron overload was not reported.

\section{Organ damage}

In children with thalassaemia, combination therapy with DFP and DFX as compared to DFP and DFO may have little or no difference in the incidence of increased creatinine, RR $3.00(99 \%$ CI 0.16 to 56.04 ) (one trial; 96 participants; low-quality evidence) (Analysis 6.4).

\section{Other adverse events (AEs) related to iron chelation}

In children with thalassaemia, we are unclear if combination therapy with DFP and DFX as compared to DFP and DFO reduces the incidence of AEs (one trial; 96 participants; very low-quality evidence) (Analysis 6.5).

- Total drug-related AEs: RR 1.08 (99\% CI 0.68 to 1.71).

- Risk of leukopenia, neutropenia, or agranulocytosis: RR

1.67 (99\% CI 0.27 to 10.14 ).

- Risk of pain or swelling in joints: RR 0.89 (99\% CI 0.29 to 2.77).

- Gastrointestinal problems: RR 0.60 (99\% CI 0.18 to 2.04).

- Liver transaminase increased: RR 1.33 (99\% CI 0.20 to 8.88).

- Skin rash: RR 5.00 (99\% CI 0.10 to 261.34).

We downgraded the quality of evidence by one for risk of bias as there was a high or unclear risk of bias in three domains; by one for indirectness, as the trial was conducted in children aged 10 to 18 with years with severe iron overload; and by one due to imprecision, the effect estimates have wide CIs.

\section{Medication management versus standard care}

One trial in thalassaemia met the inclusion criteria for this comparison (Bahnasawy 2017). See Summary of findings 7.

\section{Primary outcomes}

\section{Adherence to iron chelation therapy rates}

Adherence was only reported in the intervention group and not in the control group.

\section{Serious adverse events (SAEs)}

SAEs were not reported.

\section{All-cause mortality}

All-cause mortality was not reported.

\section{Secondary outcomes}

\section{Sustained adherence to therapy}

Adherence was only reported in the intervention group and not in the control group.

\section{Health-related quality of life (QoL)}

We are uncertain if medication management improves health-related QoL: PedsQLTM HRQoL total score median (IQR): test group: 63.51 (51.75 to 84.54); control group: 49.84 (41.9 to 60.81) (one trial; 48 participants; very low-quality evidence).

\section{Iron overload}

Proportion of participants with iron overload was not reported.

\section{Organ damage}

Proportion of participants with organ damage was not reported.

\section{Other adverse events (AEs) related to iron chelation}

AEs were not reported. 


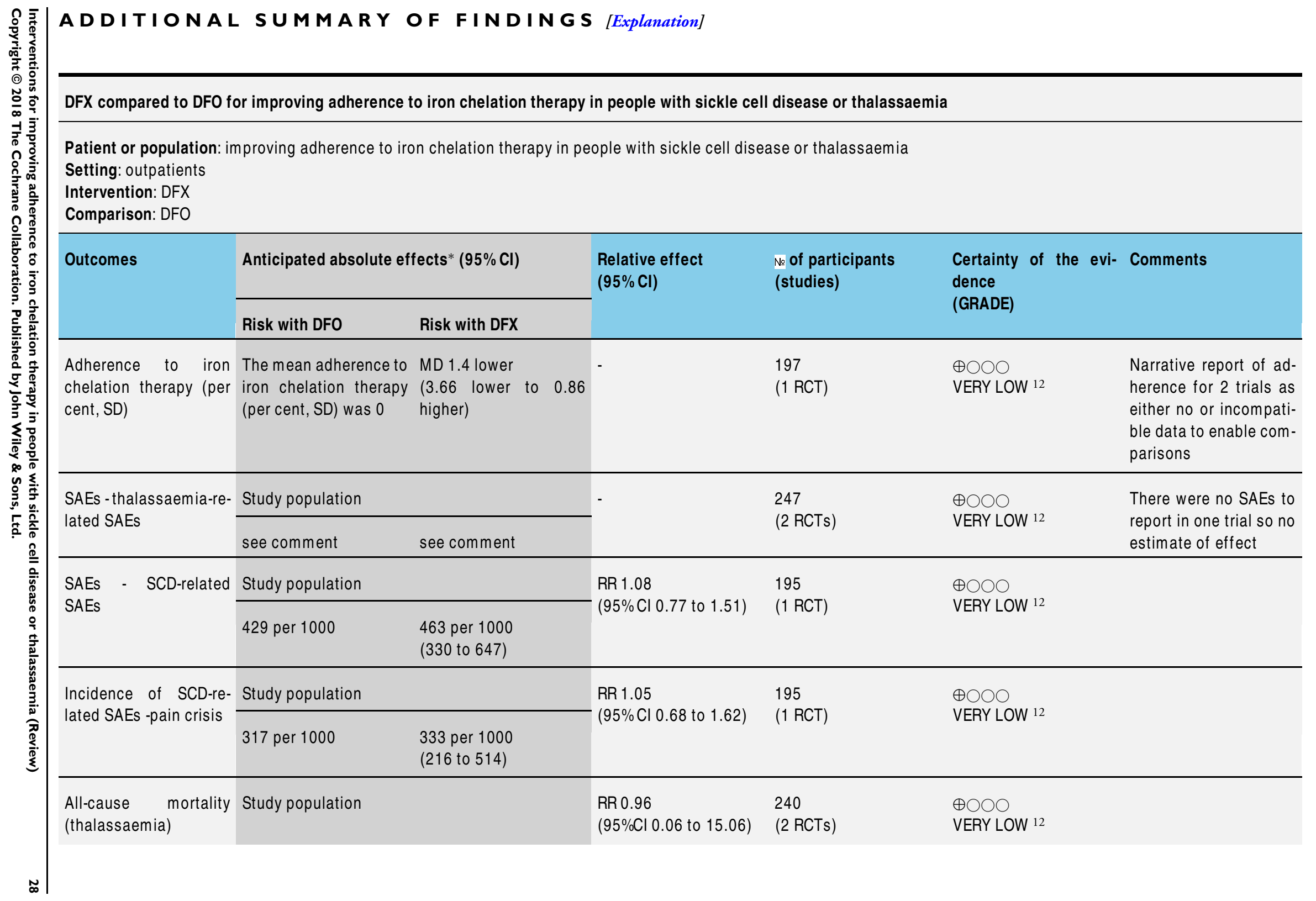




\begin{tabular}{|c|c|c|c|c|c|c|}
\hline & 8 per 1000 & $\begin{array}{l}8 \text { per } 1000 \\
(1 \text { to } 128)\end{array}$ & & & & \\
\hline $\begin{array}{l}\text { Sustained adherence - } \\
\text { not measured }\end{array}$ & - & - & - & - & - & $\begin{array}{l}\text { Sustained adherence is } \\
\text { reported as adherence } \\
\text { as all trials were longer } \\
\text { than } 6 \text { months and only } \\
\text { end of trial adherence } \\
\text { reported }\end{array}$ \\
\hline $\begin{array}{l}\text { Quality of life - not re- } \\
\text { ported }\end{array}$ & - & - & - & - & - & \\
\hline
\end{tabular}

${ }^{*}$ The risk in the intervention group (and its $95 \% \mathrm{Cl}$ ) is based on the assumed risk in the comparison group and the relative effect of the intervention (and its $95 \% \mathrm{Cl}$ ).

Cl: confidence interval; DFO: deferoxamine; DFX: deferasirox; MD: mean difference; RCT: randomised controlled trial; RR: risk ratio; SD: standard deviation

\section{GRADE Working Group grades of evidence}

High certainty: we are very confident that the true effect lies close to that of the estimate of the effect

Moderate certainty: we are moderately confident in the effect estimate: the true effect is likely to be close to the estimate of the effect, but there is a possibility that it is substantially different

Low certainty: our confidence in the effect estimate is limited: the true effect may be substantially different from the estimate of the effect

Very low certainty: we have very little confidence in the effect estimate: the true effect is likely to be substantially different from the estimate of effect

${ }^{1}$ We downgraded the quality of evidence by 2 due to high or uncertain risk of bias in several domains

${ }^{2}$ We downgraded the quality of evidence by 1 due to imprecision as $\mathrm{Cls}$ are wide and only 1 trial with data in comparison 
DFX film-coated tablet compared to DFX dispersible tablet for improving adherence to iron chelation therapy in people with sickle cell disease or thalassaemia

Patient or population: improving adherence to iron chelation therapy in people with sickle cell disease or thalassaemia

Setting: outpatients

Intervention: DFX film-coated tablet

Comparison: DFX dispersible tablet

\begin{tabular}{|c|c|c|c|c|c|c|}
\hline \multirow[t]{2}{*}{ Outcomes } & \multicolumn{2}{|c|}{ Anticipated absolute effects* $(95 \% \mathrm{Cl})$} & \multirow{2}{*}{$\begin{array}{l}\text { Relative effect } \\
(95 \% \mathrm{Cl})\end{array}$} & \multirow{2}{*}{$\begin{array}{l}\text { № of participants } \\
\text { (studies) }\end{array}$} & \multirow{2}{*}{$\begin{array}{l}\text { Certainty of the evi- } \\
\text { dence } \\
\text { (GRADE) }\end{array}$} & \multirow{2}{*}{ Comments } \\
\hline & $\begin{array}{l}\text { Risk with DFX dis- } \\
\text { persible tablet }\end{array}$ & $\begin{array}{l}\text { Risk with DFX film- } \\
\text { coated tablet }\end{array}$ & & & & \\
\hline \multirow{2}{*}{$\begin{array}{l}\text { Adherence to iron } \\
\text { chelation therapy }(n, N)\end{array}$} & Study population & & \multirow{2}{*}{$\begin{array}{l}\text { RR } 1.10 \\
-(95 \% \mathrm{Cl} 0.99 \text { to } 1.22)\end{array}$} & \multirow{2}{*}{$\begin{array}{l}173 \\
(1 \mathrm{RCT})\end{array}$} & \multirow{2}{*}{$\begin{array}{l}\oplus \oplus \circ \bigcirc \\
\text { LOW }^{1}\end{array}$} & \\
\hline & 849 per 1000 & $\begin{array}{l}934 \text { per } 1000 \\
(840 \text { to } 1000)\end{array}$ & & & & \\
\hline \multirow[t]{2}{*}{ Incidence of SAEs } & Study population & & \multirow{2}{*}{$\begin{array}{l}\text { RR } 1.22 \\
-(95 \% \mathrm{Cl} 0.62 \text { to } 2.37)\end{array}$} & \multirow{2}{*}{$\begin{array}{l}173 \\
(1 \mathrm{RCT})\end{array}$} & \multirow{2}{*}{$\begin{array}{l}\oplus \circ \bigcirc \bigcirc \\
\text { VERY LOW } 12\end{array}$} & \\
\hline & 151 per 1,000 & $\begin{array}{l}184 \text { per } 1000 \\
(94 \text { to } 358)\end{array}$ & & & & \\
\hline \multirow[t]{2}{*}{ All-cause mortality } & Study population & & \multirow{2}{*}{$\begin{array}{l}\text { RR } 2.97 \\
(95 \% \mathrm{Cl} 0.12 \text { to } 71.81)\end{array}$} & \multirow{2}{*}{$\begin{array}{l}173 \\
(1 \mathrm{RCT})\end{array}$} & \multirow{2}{*}{$\begin{array}{l}\oplus \circ \bigcirc \bigcirc \\
\text { VERY LOW } 12\end{array}$} & \\
\hline & 0 per 1000 & $\begin{array}{l}0 \text { per } 1000 \\
(0 \text { to } 0)\end{array}$ & & & & \\
\hline $\begin{array}{l}\text { Sustained adherence } \\
\text { not measured }\end{array}$ & - & - & - & - & - & $\begin{array}{l}\text { Reported as adherence } \\
\text { as trial was } 6 \text { months in } \\
\text { duration and end of trial } \\
\text { adherence reported }\end{array}$ \\
\hline
\end{tabular}

Quality of life - not re- -

ported 
${ }^{*}$ The risk in the intervention group (and its $95 \%$ confidence interval) is based on the assumed risk in the comparison group and the relative effect of the intervention (and its $95 \% \mathrm{Cl})$.

Cl: confidence interval; DFX: deferasirox; RCT: randomised controlled trial; RR: risk ratio; SAEs: serious adverse events

GRADE Working Group grades of evidence

High certainty: we are very confident that the true effect lies close to that of the estimate of the effect

Moderate certainty: we are moderately confident in the effect estimate: the true effect is likely to be close to the estimate of the effect, but there is a possibility that it is substantially different

Low certainty: our confidence in the effect estimate is limited: the true effect may be substantially different from the estimate of the effect

Very low certainty: we have very little confidence in the effect estimate: the true effect is likely to be substantially different from the estimate of effect

We downgraded the quality of evidence by 2 for risk of bias due to high or unclear risk of bias in all domains

${ }^{2}$ We downgraded the quality of evidence by 1 for imprecision due to wide $\mathrm{Cls}$ 


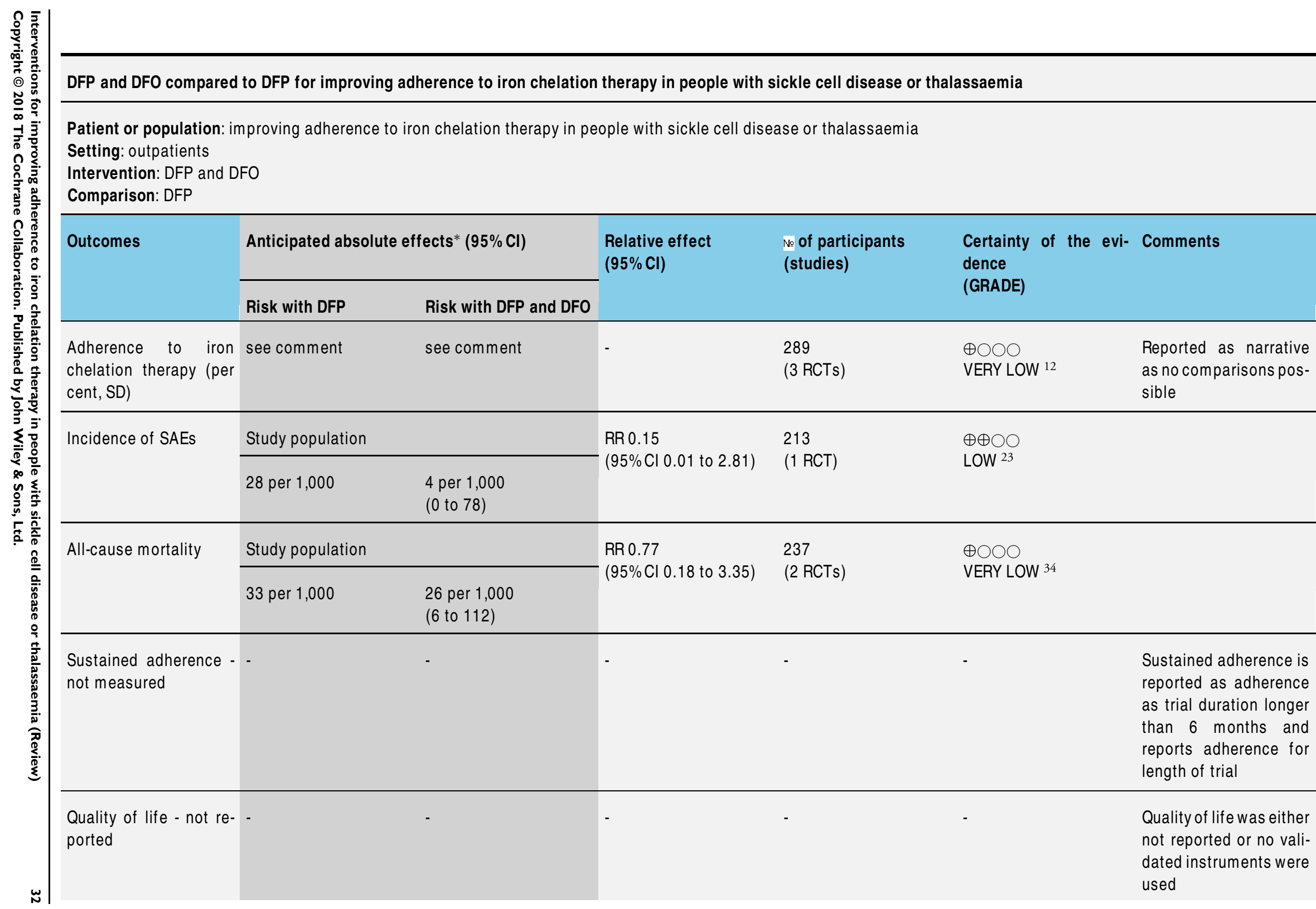


* The risk in the intervention group (and its $95 \%$ confidence interval) is based on the assumed risk in the comparison group and the relative effect of the intervention (and its $95 \% \mathrm{Cl})$.

Cl: confidence interval; DFO: deferoxamine DFP: deferiprone; RCT: randomised controlled trial; RR: risk ratio; SAEs: serious adverse events; SD: standard deviation.

GRADE Working Group grades of evidence

High certainty: we are very confident that the true effect lies close to that of the estimate of the effect

Moderate certainty: we are moderately confident in the effect estimate: The true effect is likely to be close to the estimate of the effect, but there is a possibility that it is substantially different

Low certainty: our confidence in the effect estimate is limited: The true effect may be substantially different from the estimate of the effect

Very low certainty: we have very little confidence in the effect estimate: The true effect is likely to be substantially different from the estimate of effect

We downgraded the quality of evidence by 2 for risk of bias as there was high or uncertain risk of bias in most domains in 3 out of 4 trials

${ }^{2}$ We downgraded the quality of evidence by 1 due to high or unclear risk of bias in 3 domains

3 We downgraded the quality of evidence by 1 for imprecision due to wide $\mathrm{Cls}$

${ }^{4}$ We downgraded the quality of evidence by 2 for risk of bias as there was high or uncertain risk of bias in 1 of the trials in the

comparison 


\section{DFP and DFO compared to DFO for improving adherence to iron chelation therapy in people with sickle cell disease or thalassaemi}

Patient or population: improving adherence to iron chelation therapy in people with sickle cell disease or thalassaemia

Setting: outpatients

Intervention: DFP and DFO

Comparison: DFO

\begin{tabular}{|c|c|c|c|c|c|c|}
\hline \multirow[t]{2}{*}{ Outcomes } & \multicolumn{2}{|c|}{ Anticipated absolute effects $*(95 \% \mathrm{Cl})$} & \multirow{2}{*}{$\begin{array}{l}\text { Relative effect } \\
(95 \% \mathrm{Cl})\end{array}$} & \multirow{2}{*}{$\begin{array}{l}\text { № of participants } \\
\text { (studies) }\end{array}$} & \multirow{2}{*}{$\begin{array}{l}\text { Certainty of the evi- } \\
\text { dence } \\
\text { (GRADE) }\end{array}$} & \multirow{2}{*}{ Comments } \\
\hline & Risk with DFO & Risk with DFP and DFO & & & & \\
\hline $\begin{array}{l}\text { Adherence to iron } \\
\text { chelation therapy (per } \\
\text { cent, SD) }\end{array}$ & see comment & see comment & & $\begin{array}{l}205 \\
\text { (4 RCTs) }\end{array}$ & $\begin{array}{l}\oplus \oplus \circ \bigcirc \\
\text { LOW }^{1}\end{array}$ & $\begin{array}{l}\text { Reported as narrative } \\
\text { only as adherence in } \\
\text { combined group not re- } \\
\text { ported for combination } \\
\text { therapy }\end{array}$ \\
\hline \multirow[t]{2}{*}{ Incidence of SAEs } & \multicolumn{2}{|c|}{ Study population } & & \multirow{2}{*}{$\begin{array}{l}205 \\
\text { (4 RCTs) }\end{array}$} & \multirow{2}{*}{$\begin{array}{l}\oplus \oplus \circ \bigcirc \\
\text { LOW }^{1}\end{array}$} & \multirow{2}{*}{$\begin{array}{l}3 \text { trials report no SAEs } \\
\text { SAES are not reported } \\
\text { in one trial }\end{array}$} \\
\hline & see comment & see comment & & & & \\
\hline \multirow[t]{2}{*}{ All-cause mortality } & Study populatic & & & \multirow{2}{*}{$\begin{array}{l}205 \\
\text { (4 RCTs) }\end{array}$} & \multirow{2}{*}{$\begin{array}{l}\oplus \oplus \circ \bigcirc \\
\text { LOW }^{1}\end{array}$} & \multirow[t]{2}{*}{ no deaths reported } \\
\hline & see comment & see comment & & & & \\
\hline $\begin{array}{l}\text { Sustained adherence } \\
\text { not measured }\end{array}$ & & - & & - & - & $\begin{array}{l}\text { Sustained adherence } \\
\text { reported as adherence } \\
\text { as trial duration was } \\
\text { longer than } 6 \text { months } \\
\text { and adherence reported } \\
\text { at end of trial }\end{array}$ \\
\hline
\end{tabular}

Quality of life - not re- -

ported 
${ }^{*}$ The risk in the intervention group (and its $95 \% \mathrm{Cl}$ ) is based on the assumed risk in the comparison group and the relative effect of the intervention $($ and its $95 \% \mathrm{Cl}$ ).

DFO: deferoxamine; DFP: deferiprone; SAEs: serious adverse events.

\section{GRADE Working Group grades of evidence}

High certainty: we are very confident that the true effect lies close to that of the estimate of the effect

Moderate certainty: we are moderately confident in the effect estimate: The true effect is likely to be close to the estimate of the effect, but there is a possibility that it is substantially different

Low certainty: our confidence in the effect estimate is limited: The true effect may be substantially different from the estimate of the effect

Very low certainty: we have very little confidence in the effect estimate: The true effect is likely to be substantially different from the estimate of effect

We downgraded the quality of evidence by 2 for risk of bias as high or unclear risk of bias in all domain 


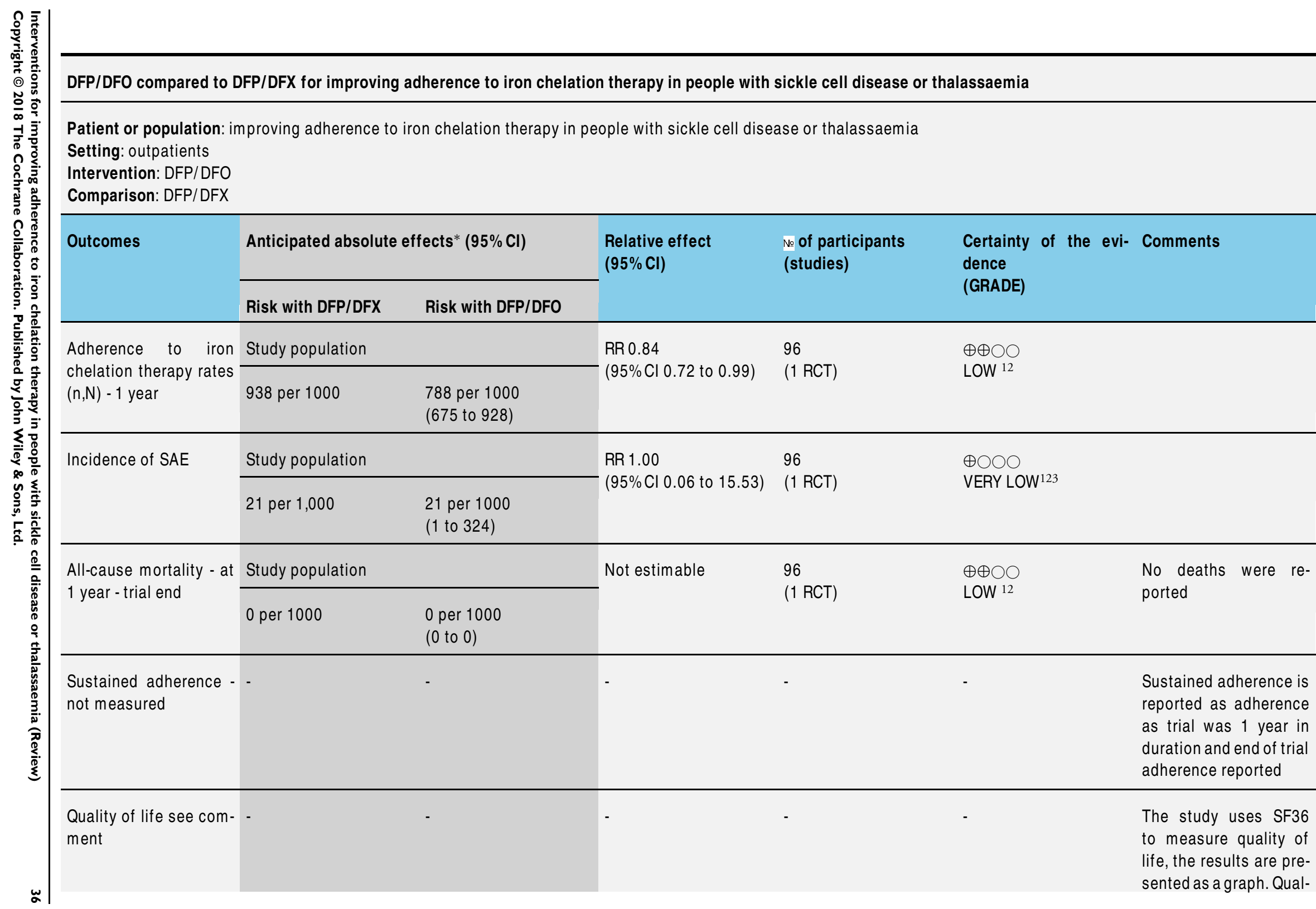


ity of life increased in both trial arms with no significant difference between trial arms $P=0.860$

${ }^{*}$ The risk in the intervention group (and its $95 \%$ confidence interval) is based on the assumed risk in the comparison group and the relative effect of the intervention (and its $95 \% \mathrm{Cl})$.

Cl: confidence interval; DFO: deferoxamine; DFP: deferiprone; DFX: deferasirox; RCT: randomised controlled trial; RR: risk ratio.

\section{GRADE Working Group grades of evidence}

High certainty: we are very confident that the true effect lies close to that of the estimate of the effect

Moderate certainty: we are moderately confident in the effect estimate: The true effect is likely to be close to the estimate of the effect, but there is a possibility that it is substantially different

Low certainty: our confidence in the effect estimate is limited: The true effect may be substantially different from the estimate of the effect

Very low certainty: we have very little confidence in the effect estimate: The true effect is likely to be substantially different from the estimate of effect

${ }^{1}$ We downgraded the quality of evidence by 1 for risk of bias as there was high or unclear risk of bias in 3 domains

2 We downgraded the quality of evidence by 1 for indirectness as the trial included children $10-18$ with severe iron overload

3 We downgraded the quality of evidence by 1 for imprecision as the comparison has wide $\mathrm{Cls}$ 
Medication management compared to standard care for improving adherence to iron chelation therapy in people with sickle cell disease or thalassaemia

Patient or population: improving adherence to iron chelation therapy in people with sickle cell disease or thalassaemia

Setting: outpatient

Intervention: medication management

Comparison: standard care

\begin{tabular}{|c|c|c|c|c|c|c|}
\hline \multirow[t]{2}{*}{ Outcomes } & \multicolumn{2}{|c|}{ Anticipated absolute effects* $(95 \% \mathrm{Cl})$} & \multirow{2}{*}{$\begin{array}{l}\text { Relative effect } \\
(95 \% \mathrm{Cl})\end{array}$} & \multirow{2}{*}{$\begin{array}{l}\text { № of participants } \\
\text { (studies) }\end{array}$} & \multirow{2}{*}{$\begin{array}{l}\text { Certainty of the evi- } \\
\text { dence } \\
\text { (GRADE) }\end{array}$} & \multirow{2}{*}{ Comments } \\
\hline & Risk with standard care & $\begin{array}{l}\text { Risk with medication } \\
\text { management }\end{array}$ & & & & \\
\hline $\begin{array}{l}\text { Adherence to iron } \\
\text { chelation therapy - not } \\
\text { reported }\end{array}$ & & & - & - & - & $\begin{array}{l}\text { Adherence was only re- } \\
\text { ported in the interven- } \\
\text { tion group and there- } \\
\text { fore no comparative } \\
\text { data }\end{array}$ \\
\hline SAEs - not reported & - & - & - & - & - & \\
\hline Mortality - not reported & - & - & - & - & - & \\
\hline Sustained adherence & - & - & - & - & - & $\begin{array}{l}\text { Adherence was only re- } \\
\text { ported in the interven- } \\
\text { tion group and there- } \\
\text { fore no comparative } \\
\text { data }\end{array}$ \\
\hline $\begin{array}{l}\text { Quality of life } \\
\text { assessed with: Ped- } \\
\text { SQLTM HRQLL total } \\
\text { score }\end{array}$ & & & - & $\begin{array}{l}48 \\
(1 \mathrm{RCT})\end{array}$ & $\begin{array}{l}\oplus \bigcirc \bigcirc \bigcirc \\
\text { VERY LOW } 12\end{array}$ & $\begin{array}{l}\text { Medication manage- } \\
\text { ment: } 63.51(51.75-84 . \\
54) \text {; standard care: } 49 . \\
84(41.9-60.81)\end{array}$ \\
\hline
\end{tabular}


${ }^{*}$ The risk in the intervention group (and its $95 \%$ confidence interval) is based on the assumed risk in the comparison group and the relative effect of the intervention (and its $95 \% \mathrm{Cl})$.

RCT: randomised controlled trial; SAEs: serious adverse events.

GRADE Working Group grades of evidence

High certainty: we are very confident that the true effect lies close to that of the estimate of the effect

Moderate certainty: we are moderately confident in the effect estimate: The true effect is likely to be close to the estimate of the effect, but there is a possibility that it is substantially different

Low certainty: our confidence in the effect estimate is limited: The true effect may be substantially different from the estimate of the effect

Very low certainty: we have very little confidence in the effect estimate: The true effect is likely to be substantially different from the estimate of effect

We downgraded the quality of evidence for indirectness by 2 because most outcomes were only reported in the medication

management group

${ }^{2}$ We downgraded the quality of evidence by 2 for risk of bias due to high or uncertain risk of bias in all domains 


\section{I SCUSSION}

Regularly transfused people with SCD, as well as transfusiondependent, and non-transfusion-dependent people with thalassaemia, are at risk of iron overload. Iron overload can lead to iron toxicity, with organs such as the heart, liver and endocrine glands being particularly vulnerable.

In this review we reviewed the evidence for improving adherence to iron chelation therapy in people with SCD or thalassaemia.

Sixteen RCTs with a total of 1525 participants met our inclusion criteria. Fourteen trials included people with $\beta$-thalassaemia major, one trial was conducted in people with SCD and another in people with $\beta$-thalassaemia intermedia, a milder form of $\beta$ thalassaemia. Trials were conducted between 1997 and 2017 and all included trials were medication interventions, except for one, which was a medication management intervention.

We also identified an additional five ongoing RCTs, and two studies awaiting classification (one RCT and one prospective cohort study).

We did not identify any cluster randomised trials, NRSIs, CBA or ITS studies that met the inclusion criteria.

\section{Summary of main results}

The findings of the review led to the following main conclusions regarding medication interventions to improve adherence to iron chelation.

\section{DFP versus DFO}

Based on results from four trials in thalassaemia, we are uncertain whether oral DFP increases adherence to iron chelation therapy more than subcutaneous DFO (Calvaruso 2015; El Beshlawy 2008; Olivieri 1997; Pennell 2006). Results could not be combined due to a lack of data to report as well as the considerable heterogeneity between comparisons $\left(\mathrm{I}^{2}=99 \%\right)$. There was high adherence in all trials. We are uncertain if switching to oral DFP increases the risk of agranulocytosis compared to subcutaneous DFO. Oral DFP may have little or no effect on mortality compared to subcutaneous DFO. Quality of life was not measured in any trial in this comparison.

\section{DFX versus DFO}

Based on results from three trials, two in thalassaemia (Hassan 2016; Pennell 2014) and one in SCD (Vichinsky 2007), we are uncertain if DFX increases the rate of adherence compared to people taking DFO; participants had high adherence in all trials. We are uncertain whether DFX decreases risk of thalassaemiarelated SAEs or decreases the risk of mortality in people with thalassaemia compared to DFO. We are uncertain whether DFX decreases the risk of SCD-related pain crisis or other SCD-related SAEs compared to DFO. QoL was not reported in any trial in this comparison.

\section{DFX (film-coated tablet (FCT)) versus DFX (dispersible tablet (DT))}

Based on results from a single trial in thalassaemia, DFX FCT may make little or no difference to adherence as compared to DFX DT (Taher 2017). There was high adherence in both arms of the trial. We are uncertain if DFX FCT increases SAEs or all-cause mortality as compared to DFX DT. QoL was not measured using a validated instrument.

\section{DFP and DFO combined versus DFP alone}

Based on results from three trials in thalassaemia, we are uncertain if DFP and DFO combined increases adherence compared to DFP alone (Aydinok 2007; El Beshlawy 2008; Maggio 2009). There was high adherence in all trials. Combination therapy with DFP and DFO may make little or no difference to the incidence of SAEs as compared to DFP alone. We are uncertain if combination therapy with DFP and DFO decreases mortality as compared to DFP alone. QoL was not measured using a validated instrument.

\section{DFP and DFO combined versus DFO alone}

Based on results from four trials in people with thalassaemia, combined therapy with DFP and DFO versus DFO alone, may make little or no difference to adherence rates, SAEs, or mortality (El Beshlawy 2008; Galanello 2006; Mourad 2003; Tanner 2007). There was high adherence in all trials. QoL was not measured in any trial in this comparison.

\section{DFP and DFO combined versus DFP and DFX combined}

Based on the results of a single trial in children with thalassaemia, combination therapy with DFP and DFX may improve adherence to iron chelation therapy compared to combination therapy with DFP and DFO (Elalfy 2015). There was high adherence in both arms. We are uncertain if DFP and DFX reduces the incidence of SAEs, and may make little or no difference in mortality or QoL, compared to combination therapy with DFP and DFO.

\section{Medication management versus standard care}

A single trial on thalassaemia reported on this comparison (Bahnasawy 2017). Adherence rates were only reported in the intervention arm and therefore there are no comparative data to report. We are uncertain if medication management improves health-related QoL. 


\section{Overall completeness and applicability of evidence}

This review provides the most up-to-date assessment of interventions to improve adherence to iron chelation therapy in people with SCD and thalassaemia. We have also identified five ongoing trials and two trials that are awaiting classification.

Of the five ongoing trials, two compare medication interventions in thalassaemia and two in SCD and thalassaemia (EudraCT 2012-000353-31; IRCT2015101218603N2; NCT02173951; NCT02435212), and one assesses the effectiveness of group medical appointments on self-efficacy and adherence in SCD (Madderom 2016). Of the two studies awaiting classification, one is an educational study (Antmen 2013), and one is a medication intervention (NCT00004982).

The results of this review can only be interpreted in consideration of the following factors.

- Adherence is not the primary outcome in any of the included trials.

- All trials, except for one medication management trial, are medication interventions and participants were often selected based on their anticipated compliance. Lack of adherence was a reason for exclusion from some trials or analyses of results.

- Within the context of a clinical trial, there is increased attention by, and involvement of, clinicians and specialist nurses with participants which may impact and increase rates of adherence not seen in a community setting.

- Research has shown that up to $50 \%$ of people do not take medications as prescribed and over $85 \%$ of people are occasionally non-adherent to prescribed medications (Ryan 2014). The reported adherence rates in the trials included in this review are substantially higher than average, despite the substantial side effects and demanding administration regimen of iron chelators. This may be indicative of high adherence rates being an artefact created by participant involvement in a clinical trial.

- We did not identify any cluster randomised trials, NRSIs, CBA or ITS studies with adherence as a primary outcome, that met the inclusion criteria.

- Due to a lack of evidence this review cannot comment on intervention strategies for different age groups.

\section{Quality of the evidence}

Overall the quality of the evidence according to GRADE methodology across all comparisons for the outcomes of adherence, SAEs, and mortality was rated as low to very low (Summary of findings for the main comparison; Summary of findings 2; Summary of findings 3; Summary of findings 4; Summary of findings 5; Summary of findings 6; Summary of findings 7). This was due to trials being at serious or very serious risk of bias; outcome estimates being imprecise (wide CIs); and indirectness with some trials conducted only in children of a specific age and meeting specific criteria. QoL was mostly not reported or reported using non-validated measurements or sparsely reported with no data.

\section{Potential biases in the review process}

To our knowledge, our review process was free from bias. We conducted a comprehensive search: searching data sources (including multiple databases, and clinical trial registries) to ensure that all relevant studies would be captured. There were no restrictions for the language in which the paper was originally published. The relevance of each paper was carefully assessed and all screening and data extractions were performed in duplicate. We pre-specified all outcomes and subgroups prior to analysis. There were insufficient numbers of included trials within the meta-analyses for us to use a funnel plot to examine the risk of publication bias.

\section{Agreements and disagreements with other studies or reviews}

Adherence rates can vary widely, a recent review reported that adherence rates to the oral iron chelator DFX ranged between 22\% and $89 \%$ (Loiselle 2016). Another review of medication adherence in SCD reports adherence rates ranging from $16 \%$ to $89 \%$; but most included studies reported moderate adherences (Walsh 2014). In this Cochrane Review, we found adherence rates across trials and for all comparisons of different chelators to be quite high in individual trial reports (predominantly at least 80\%). Indeed the results of this review are in disagreement with most literature that identifies major issues with compliance across indications, people and setting (NICE 2009; Ryan 2014; WHO 2003). We suggest that selection bias for compliance into the chelation trials as a possible reason for high adherence; as well, the additional time and attention received by participants make high adherence an artefact of trial participation.

Ryan identifies several strategies that may help to promote adherence including self-management; self-monitoring; simplified dosing regimens; or interventions involving pharmacists in medication management (Ryan 2014). Other identified interventions that need further research include pragmatic interventions (such as reminders); educational interventions, and financial incentives. One RCT on pharmacist-led medication management was included in this review, but the trial had few participants, was of short duration and poorly reported (Bahnasawy 2017). The remaining trials in this review measured compliance primarily as a secondary outcome and did not identify any specific strategies that may have led to increased compliance, thus supporting the contention that high compliance is an artefact of participation in these trials and not the result of change or improvement in medication regimens. 


\section{AUTHORS' CONCLUSIONS}

\section{Implications for practice}

Adherence to iron chelation regimens can reduce morbidity and mortality in people with transfusion- and non-transfusion-dependent thalassaemia and sickle cell disease. Iron chelation regimens can be demanding and also have unpleasant side effects that reduce adherence to these medications. In this review we did not identify any specific medication intervention that increased adherence with iron chelators and suggest that adherence was high due to the artefact of participation in these trials. Due to a lack of evidence, this review cannot comment on intervention strategies for different age groups.

Overviews of systematic reviews that identify intervention strategies that have been successful for other indications and medications may be more useful to clinicians who want to improve compliance with iron chelation therapy. However, the successful translation of these interventions to iron chelation regimens would still need to be confirmed in appropriate trials.

\section{Implications for research}

Real-world, pragmatic trials in community and clinic settings are needed to examine a variety of confirmed or unconfirmed adherence strategies that may be useful to increase adherence to iron chelation therapy. High-quality, non-randomised trials that measure compliance over multiple time points, before and after an intervention, as well as non-randomised studies that test interventions in multiple settings could help to identify evidence-based strategies that increase compliance with iron chelation therapy. Finally, appropriate measurements of compliance are needed that include both patient-oriented, such as quality of life measurements, as well as objective measurements that link iron levels and morbidity due to iron overload to levels of adherence. Targeted strategies that increase adherence in different age groups, particularly in adolescents, are also needed.

\section{ACKNOWLEDGEMENTS}

We thank the National Institute for Health Research for supporting this project, via Cochrane Infrastructure funding to the Cochrane Cystic Fibrosis and Genetic Disorders Group. The views and opinions expressed therein are those of the authors and do not necessarily reflect those of the Systematic Reviews Programme, NIHR, NHS or the Department of Health.

This review is part of a series of reviews that have been partly funded by the NIHR Cochrane Programme Grant - Safe and Appropriate Use of Blood Components.

\section{R E F E R E N C E S}

\section{References to studies included in this review}

\section{Aydinok 2007 \{published data only\}}

Aydinok Y, El-Beshlawy A, von Orelli-Leber C, CzarneckiTarabishi C, Manz C Y. A randomized controlled trial comparing the combination therapy of deferiprone (DFP) and desferrioxamine (DFO) versus DFP or DFO monotherapy in patients with thalassemia major. Blood 2006;108(11). Abstract no.: 557]

* Aydinok Y, Ulger Z, Nart D, Terzi A, Cetiner N, Ellis G, et al. A randomized controlled 1-year study of daily deferiprone plus twice weekly desferrioxamine compared with daily deferiprone monotherapy in patients with thalassemia major. Haematologica 2007;92(12):1599-606. Manz CH, El-Beshlawy A, Aydinok Y, Leber C, CzarneckiTarabishi C. A randomized controlled prospective clinical study comparing the combination therapy of deferiprone (L1) and desferrioxamine with L1 and DFO monotherapy in patients with thalassemia major. Haematologica 2006;91 (S1):190. Abstract no.: 0515]

Badawy 2010 \{published data only\}

Badawy S, Hassan TH, Hesham MA, Badr MA. Evaluation of iron chelation therapy in B-thalassemic patients in Zagazig University Hospital. ASPHO abstracts (The
American Society of Pediatric Hematology/Oncology) 2010; Vol. 54, issue 6:799-800.

Bahnasawy 2017 \{published data only\} Bahnasawy SM, El Wakeel LM, El Beblawy N, El-Hamamsy $\mathrm{M}$. Clinical pharmacist-provided services in iron overloaded Beta-thalassemia major children; A new insight to patient care. Basic \& Clinical Pharmacology \& Toxicology 2017;120 (4):354-9.

Calvaruso 2015 \{published data only\} * Calvaruso G, Vitrano A, Di Maggio R, Ballas S, Steinberg $\mathrm{MH}$, Rigano P, et al. Deferiprone versus deferoxamine in thalassemia intermedia: results from a 5-year long-term Italian multicenter randomized clinical trial. American Journal of Hematology 2015;90(7):634-8.

Vitrano A, Calvaruso G, Di Maggio G, Romeo MA, Cianciulli P, Lai ME, et al. Deferiprone versus deferoxamine in thalassemia intermedia: results from 5-year long-term Italian multi-center randomized clinical trial. 56th ASH Annual Meeting and Exposition; 2014 Dec 6-9; San Francisco, California 2014.

Elalfy 2015 \{published data only\} Elalfy M, Walli Y, Adly A, Henawy Y. 18 months data of a randomized controlled trial of combined deferiprone (DFP) and deferasirox (DFX) versus combined deferiprone 
and deferoxamine (DFO), in young B-thalassemia major. Haematologica 2014;99(S1):443-4.

* Elalfy MS, Adly AM, Wali Y, Tony S, Samir A, Elhenawy YI. Efficacy and safety of a novel combination of two oral chelators deferasirox/deferiprone over deferoxamine/ deferiprone in severely iron overloaded young beta thalassemia major patients. European Journal of Haematology 2015;95(5):411-20.

Elalfy MS, Wali Y, Tony S, Samir, Adly A. Comparison of two combination iron chelation regimens, deferiprone and deferasirox versus deferiprone and deferoxamine, in pediatric patients with beta-thalassemia major. Blood 2013; 122(21). Abstract no.: 559]

\section{El Beshlawy 2008 \{published data only\}}

Study With Deferiprone and/or Desferrioxamine in Iron Overloaded Patients. ClinicalTrials.gov 2006; Vol. NCT00350662.

El-Beshlawy A, Manz C, Naja M, Eltagui M, Tarabishi C, Youssry I, et al. Iron chelation in thalassemia: combined or monotherapy? The Egyptian experience. Annals of Hematology 2008;87(7):545-50.

\section{Galanello 2006 \{published data only\}}

Galanello R, Kattamis A, Piga A, Tricta F. Safety and efficacy of alternate desferrioxamine and deferiprone compared to desferrioxamine alone in the treatment of iron overload in transfusion-dependent thalassemia patients. Blood 2004; 104(11 Pt 1). Abstract no.: 3611]

* Galanello R, Piga A, Forni G L, Bertrand Y, Foschini M L, Bordone E, et al. Phase II clinical evaluation of deferasirox, a once-daily oral chelating agent, in pediatric patients with beta-thalassemia major. Haematologica 2006;91(10): 1343-51.

\section{Hassan 2016 \{published data only\}}

Hassan MA, Tolba OA. Iron chelation monotherapy in transfusion-dependent beta-thalassemia major patients: A comparative study of deferasirox and deferoxamine. Electronic physician 2016;8(5):2425-2431.

Maggio 2009 \{published data only\}

Maggio A, Capra M, Cuccia L, Gagliardotto F, Magnano C, Caruso V, et al. Deferiprone versus sequential deferipronedeferoxamine treatment in thalassemia major: a five years multicenter randomized clinical trial under the auspices of the society for the study of thalassemia and hemogobinopathies (SoST). Blood 2007;110(11). Abstract no.: 575]

Maggio A, Vitrano A, Capra M, Cuccia L, Gagliardotto F, Filosa A, et al. Decrease of mortality during deferiprone treatments: results from a large randomised cohort of thalassemia major patients under the auspices of the Italian society for thalassemia and hemogobinopathies. Blood 2008;112 Suppl:Abstract no: 3885.

* Maggio A, Vitrano A, Capra M, Cuccia L, Gagliardotto F, Filosa A, et al. Long-term sequential deferipronedeferoxamine versus deferiprone alone for thalassaemia major patients: A randomized clinical trial. British Journal of Haematology 2009;145(2):245-54.

NCT00733811. Efficacy Study of the Use of Sequential DFP-DFO Versus DFP. https://clinicaltrials.gov/ct2/show/ NCT00733811 (accessed 11 April 2018).

Pantalone GR, Maggio A, Vitrano A, Capra M, Cuccia L, Gagliardotto F, et al. Sequential alternating deferiprone and deferoxamine treatment compared to deferiprone monotherapy: Main findings and clinical follow-up of a large multicenter randomized clinical trial in -thalassemia major patients. Hemoglobin 2011;35(3):206-16.

Mourad 2003 \{published data only\}

Mourad FH, Hoffbrand AV, Sheikh-Taha M, Koussa S, Khoriaty AI, Taher A. Comparison between desferrioxamine and combined therapy with desferrioxamine and deferiprone in iron overloaded thalassaemia patients. British Journal of Haematology 2003;121(1):187-9.

\section{Olivieri 1997 \{published data only\}}

Olivieri N, the Iron Chelation Research Group. Randomized trial of deferiprone (LI) and deferoxamine (DFO) in thalassemia major. Blood 1996; Vol. 88, issue 10 Suppl 1:651a.

Olivieri NF, Brittenham GM. Evidence of progression of myocardial iron loading as determined by magnetic resonance imaging (MRI) in thalassemia patients during treatment with deferiprone (L1) and deferoxamine (DFO). Blood 1999; Vol. 94, issue 10 Suppl 1:35b.

* Olivieri NF, Brittenham GM. Final results of the randomized trial of deferiprone (L1) and deferoxamine (DFO). Blood 1997; Vol. 90, issue 10 Suppl 1:264a. Olivieri NF, Brittenham GM, Armstrong SAM, Basran RK, Daneman R, Daneman N, et al. First prospective randomized trial of the iron chelators deferiprone (L1) and deferoxamine. Blood 1995; Vol. 86, issue 10 Suppl 1:249a. Pope, Elena. Critical review of standard and new methods of assessing compliance with chelation therapy in thalassemic patients. Proquest Dissertations Publishing 1995.

\section{Pennell 2006 \{published data only\}}

* Pennell D J, Berdoukas V, Karagiorga M, Ladis V, Piga A, Aessopos A, et al. Randomized controlled trial of deferiprone or deferoxamine in beta-thalassemia major patients with asymptomatic myocardial siderosis. Blood 2006;107(9):3738-44.

Smith GC, Alpendurada F, Carpenter JP, Alam MH, Berdoukas V, Kargiorga M, et al. Effect of deferiprone or deferoxamine on right ventricular function in thalassemia major patients with myocardial iron overload. Journal of Cardiovascular Magnetic Resonance 2011; Vol. 13, issue 1: 34.

\section{Pennell 2014 \{published data only\}}

Aydinok Y, Porter JB, Piga A, Elalfy M, El-Beshlawy A, Kilinc Y, et al. Prevalence and distribution of iron overload in patients with transfusion-dependent anemias differs across geographic regions: results from the CORDELIA 
study. European Journal of Haematology 2015;95(3): 244-53.

Pennell D, Porter J, Piga A, El-Alfy M, El-Beshlawy A, Kilinc Y, et al. Prevalence of cardiac iron overload in patients with transfusion-dependent anemias: data from the randomized, active-controlled deferasirox CORDELIA trial. Haematologica 2012;97 Suppl 1:384. Abstract no.: 0928]

Pennell D, Porter JB, Piga A, Lai Y, El-Beshlawy A, Beloul $\mathrm{K}$, et al. A multicenter, randomized, open-label trial evaluating deferasirox compared with deferoxamine for the removal of cardiac iron in patients with beta-thalassemia major and iron overload (CORDELIA). Blood 2012;120 (21). Abstract no.: 2124]

* Pennell DJ, Porter JB, Piga A, Lai Y, El-Beshlawy A, Belhoul K M, et al. A 1-year randomized controlled trial of deferasirox vs deferoxamine for myocardial iron removal in beta-thalassemia major (CORDELIA). Blood 2014;123 (10): 1447-54.

Taher 2017 \{published data only\}

A randomized, open-label, multicenter, two arm, phase II study to investigate the benefits of an improved deferasirox formulation (Film-coated Tablet). ClinicalTrials.go 2014; Vol. NCT02125877.

A randomized, open-label, multicenter, two arm, phase II study to investigate the benefits of an improved deferasirox formulation (film-coated tablet). EU Clinical Trials Register issue https://www.clinicaltrialsregister.eu/ctr-search/trial/ 2013-004167-32/AT.

Huang VW, Banderas B, Sen R. Psychometric evaluation of clinical outcomes assessments in a phase II trial. Value in Health 2016; Vol. 19, issue 7:A746.

* Taher AT, Origa R, Perrotta S, Kourakli A, Ruffo GB, Kattamis A, et al. New film-coated tablet formulaion of deferasirox is well tolerated in patients with thalassemia or lower-risk MDS: Results of the randomized, Phase II ECLIPSE study. American Journal of Hematology 2017;92 (5):420-8.

Tanner 2007 \{published data only\}

Tanner MA, Galanello R, Dessi C, Agus A, Smith GC, Westwood MA, et al. Improved endothelial function combined chelation therapy in thalassaemia major. Blood 2006;108(11). Abstract no.: 1770]

* Tanner MA, Galanello R, Dessi C, Smith GC, Westwood MA, Agus A, et al. A randomized, placebo-controlled, double-blind trial of the effect of combined therapy with deferoxamine and deferiprone on myocardial iron in thalassemia major using cardiovascular magnetic resonance. Circulation 2007;115(14):1876-84.

Tanner MA, Galanello R, Dessi C, Smith GC, Westwood MA, Agus A, et al. The effect of combined therapy with deferoxamine and deferiprone on myocardial iron and endothelial function in thalassaemia major: a randomized controlled trials using cardiovascular magnetic resonance. Haematologica 2006; Vol. 91, issue Suppl 1:191. Tanner MA, Galanello R, Dessi C, Westwood MA, Smith GC, Khan M, et al. A randomized, placebo controlled, double blind trial of the effect of combined therapy with deferoxamine and deferiprone on myocardial iron in thalassaemia major using cardiovascular magnetic resonance. Blood 2005;106(11 Pt 1). Abstract no.: 3655]

\section{Vichinsky 2007 \{published data only\}}

Vichinsky E. Patient reported outcomes with chelation therapy in patients with sickle cell disease (SCD) on either deferasirox (Exjade®, ICL670) or deferoxamine (DFO). 29th annual meeting of the National Sickle Cell Disease Program; April 8-12; Memphis, USA 2006. Abstract no.: 174]

Vichinsky E. Results of a randomized, controlled phase two trials of deferasirox (Exjade ${ }^{\circledR}$, ICL670) in sickle cell disease patients with chronic overload. 29th Annual Meeting of the National Sickle Cell Disease Program; April 8-12; Memphis, USA 2006. Abstract no.: 175]

Vichinsky E, Bernaudin F, Forni GL, Gardner R, Hassell $\mathrm{K}$, Heeney MM, et al. Long-term safety and efficacy of deferasirox (Exjade) for up to 5 years in transfusional ironoverloaded patients with sickle cell disease. British Journal of Haematology 2011;154(3):387-97.

Vichinsky E, Bernaudin F, Forni GL, Gardner R, Hassell KL, Heeney MM, et al. Long-term safety and efficacy of deferasirox (Exjade $\left.{ }^{\circledR}\right)$ in transfused patients with sickle cell disease treated for up to 5 years. Blood 2011;118(21). Abstract no.: 845]

Vichinsky E, Coates T, Thompson A, Bernaudin F, Lagrone $\mathrm{D}$, Dong V, et al. Safety and efficacy of iron chelation therapy with deferasirox in patients with sickle cell disease (SCD): 3.5-year follow-up. 14th Congress of the European Haematology Association; Jun 4-7; Berlin, Germany 2009. Vichinsky E, Coates T, Thompson AA, Bernaudin F, Rodriguez M, Rojkjaer L, et al. Deferasirox (Exjade $\left.{ }^{\circledR}\right)$, the once-daily oral iron chelator, demonstrates safety and efficacy in patients with sickle cell disease (SCD): 3.5-year follow-up. Blood 2008;112(11). Abstract no.: 1420] Vichinsky E, Coates T, Thompson AA, Bernaudin F, Rodriguez M, Rojkjaer L, et al. Deferasorix (Exjade®), the once-daily oral iron chelator, demonstrates safety and efficacy in patients with sickle cell disease (SCD): 3.5-year follow-up. 3rd Annual Sickle Cell Disease Research and Educational Symposium and Annual Sickle Cell Disease Scientific Meeting; Feb 18-20; Florida, USA 2009. Abstract no.: 225]

Vichinsky E, Coates T, Thompson AA, Mueller BU, Lagrone D, Heeney MM. Long-term efficacy and safety of deferasirox (Exjade®, ICL670), a once-daily oral iron chelator, in patients with sickle cell disease (SCD). Blood 2007;110(11 Pt 1):995A. Abstract no.: 3395]

Vichinsky E, Fischer R, Fung E, Onyekwere O, Porter J, Swerdlow P, et al. A randomized, controlled phase two trial in sickle cell disease patients with chronic iron overload demonstrates that the once-daily oral iron chelator deferasirox (Exjade®, ICL670) is well tolerated and reduces iron burden. Blood 2005;106. Abstract no.: 313] Vichinsky E, Fischer R, Pakbaz Z, Onyekwere O, Porter J, Swerdlow P, et al. Satisfaction and convenience of 
chelation therapy in patients with sickle cell disease (SCD): Comparison between deferasirox (Exjade®, ICL670) and deferoxamine (DFO). Blood 2005;106(11 Pt 1). Abstract no.: 2334]

* Vichinsky E, Onyekwere O, Porter J, Swerdlow P, Eckman J, Lane P, et al. A randomised comparison of deferasirox versus deferoxamine for the treatment of transfusional iron overload in sickle cell disease. British Journal of Haematology 2007;136(3):501-8.

Vichinsky E, Pakbaz Z, Onyekwere O, Porter J, Swerdlow $P$, Coates T, et al. Patient-reported outcomes of deferasirox (Exjade, ICL670) versus deferoxamine in sickle cell disease patients with transfusional hemosiderosis. Substudy of a randomized open-label phase II trial. Acta Haematologica 2008;119(3):133-141.

\section{References to studies excluded from this review}

Abu 2015 \{published data only\}

Abu SO, Auda W, Kamhawy H, Al-Tonbary Y. Impact of educational programme regarding chelation therapy on the quality of life for B-thalassemia major children. Hematology 2015;20(5):297-303.

Al Kloub 2014 \{published data only\} Al-Kloub MI, A Bed MA, Al Khawaldeh OA, Al Tawarah YM, Froelicher ES. Predictors of non-adherence to followup visits and deferasirox chelation therapy among Jordanian adolescents with Thalassemia major. Pediatric Hematology and Oncology 2014;31(7):624-37.

Al Kloub 2014a \{published data only\}

Al-Kloub, MI, TN, Salameh, ES, Froelicher. Impact of psychosocial status and disease knowledge on deferoxamine adherence among thalassaemia major adolescents. International Journal of Nursing Practice 2014;20(3): 265-74.

Al Refaie 1995 \{published data only\}

Al-Refaie FN, Hershko C, Hoffbrand AV, Kosaryan M, Olivier NF, Tondury P, et al. Results of long-term deferiprone (L1) therapy: a report by the International Study Group on Oral Iron Chelators. British Journal of Haematology 1995;91(1):224-9.

Alvarez 2009 \{published data only\} Alvarez O, Rodriguez-Cortes H, Robinson N, Lewis N, Pow Sang CD, Lopez-Mitnik G, et al. Adherence to deferasirox in children and adolescents with sickle cell disease during 1-year of therapy. Journal of Pediatric Hematology/Oncology 2009;31(10):739-44.

Armstrong 2011 \{published data only\} Armstrong EP, Skrepnek GH, Ballas SK, Kwok P, Snodgrass S, Sasane M. Costs, persistence, and hospitalizations associated with the use of iron-chelating therapies in sickle cell disease in medicaid patients. Blood 2011; Vol. 118, issue 21.

Bala 2014 \{published data only\}

Bala J, Sarin J. Treatment adherence and quality of life of thalassemic children. International Journal of Nursing Education 2014;6(2):151-2.
Belgrave 1989 \{published data only\}

Belgrave FZ, Gilbert SK. Health care adherence of persons with sickle cell disease. The role of social support. Annals of the New York Academy of Sciences 1989;565:369-70.

\section{Berkovitch 1995 \{published data only\}} Berkovitch M, Davis S, Matsui D, DonskyJ, Koren G, Olivieri NF. Use of a eutectic mixture of local anesthetics for prolonged subcutaneous drug administration. Journal of Clinical Pharmacology 1995;35(3):295-7.

\section{Chakrabarti 2013 \{published data only\}}

Chakrabarti P, Bohara V, Ray S, Sankar Ray S, Kumar, NU, Chaudhuri U. Can the availability of unrestricted financial support improve the quality of care of thalassemics in a center with limited resources? A single center study from India. Thalassemia Reports 2013;3(1):6-10.

Daar 2010 \{published data only\} Daar S, Al, Salmi F, Ableen V, Jacob W, Jabeen Z, Pathare A. T2*MRI - An effective tool to increase chelation compliance in thalassemia major. Haematologica 2010, issue 95:698.

\section{Gomber 2004 \{published data only\}} Gomber S, Saxena R, Madan N. Comparative efficacy of desferrioxamine, deferiprone and in combination on iron chelation in thalassemic children. Indian Pediatrics 2004;41 (1):21-7.

\section{Kidson Gerber 2008 \{published data only\}}

Kidson-Gerber G, Lindeman R. Adherence to desferrioxamine and deferiprone and the impact of deferiprone co-prescription in thalassaemia major patients. Does the addition of deferiprone improve adherence?. British Journal of Haematology 2008;142(4):679-80.

Kolnagou 2008 \{published data only\} Kolnagou A, Economides C, Eracleous E, Kontoghiorghes GJ. Long term comparative studies in thalassemia patients treated with deferoxamine or a deferoxamine/deferiprone combination. Identification of effective chelation therapy protocols. Hemoglobin 2008;32(1-2):41-7.

\section{Leonard 2014 \{published data only\}}

Leonard S, Jonassaint J, Anderson L, Shah N. The use of mobile technology for intensive training in medication management in the pediatric population. Blood 2014; Vol. 124 , issue 21.

Loiselle 2016 \{published data only\} Loiselle K, Lee JL, Szulczewski L, Drake S, Crosby LE, Pai AL. Systematic and meta-analytic review: medication adherence among pediatric patients With sickle cell disease. Journal of Pediatric Psychology 2016;41(4):406-18.

Mazzone 2009 \{published data only\} Mazzone L, Battaglia L, Andreozzi F, Romeo MA, Mazzone D. Emotional impact in beta-thalassaemia major children following cognitive-behavioural family therapy and quality of life of caregiving mothers. Clinical Practice and Epidemiology in Mental Health 2009;5(5):Online.

NCT01709032 \{published data only\} NCT01709032. Combination deferasirox and deferiprone for severe iron overload in thalassemia. https:// 
clinicaltrials.gov/ct2/show/NCT01709032 (accessed 11

April 2018).

\section{NCT01825512 \{published data only\}}

NCT01825512. Multicentre, randomised, open label, noninferiority active-controlled trial to evaluate the efficacy and safety of deferiprone compared to deferasirox in paediatric patients aged from 1 month to less than 18 years of age affected by transfusion-dependent haemoglobinopathies. https://clinicaltrials.gov/ct2/show/NCT01825512 (accessed 11 April 2018).

\section{NCT02133560 \{published data only\}}

NCT02133560. Use of mobile technology for intensive training in medication management. https:// clinicaltrials.gov/ct2/show/NCT02133560 (accessed 14 April 2018).

NCT02466555 \{published data only\}

NCT02466555. Music Therapy in Sickle Cell Transition Study. https://clinicaltrials.gov/ct2/show/NCT02466555 (accessed 14 April 2018). Sponsors: University Hospital Case Medical Center|Kulas FoundationOther IDs: 03-15-30]

Pakbaz 2004 \{published data only\} Pakbaz Z, Fischer R, Gamino R, Quirolo K, Yamashita R, Treadwell $\mathrm{M}$, et al. Assessing compliance to iron chelation therapy in patients with thalassemia. Blood 2004; Vol. 104, issue 11:33B.

Pakbaz 2005 \{published data only\}

Pakbaz ZR, Fischer M, Treadwell R, Yamashita EB, Fung $\mathrm{L}$, Calvell K, et al. A simple model to assess and improve adherence to iron chelation therapy with deferoxamine in patients with thalassemia. Annals of the New York Academy of Sciences 2005;1054:486-91.

Porter 2009 \{published data only\}

Porter JB, Athanasiou-Metaxa M, Bowden DK, Troncy, J, Habr D, Domokos G, et al. Improved patient satisfaction, adherence and health-related quality of life with deferasirox (Exjade) in beta-thalassemia patients previously receiving other iron chelation therapies. Blood 2009; Vol. 114, issue 22.

Porter 2012 \{published data only\}

Porter J, Bowden DK, Economou M, Troncy J, Ganser A, Habr D, et al. Health- related quality of life, treatment satisfaction, adherence and persistence in beta-thalassemia and myelodysplastic syndrome patients with iron overload receiving deferasirox: results from the epic clinical trial. Anemia 2012;297:641.

Vichinsky 2005 \{published data only\} Vichinsky E, Fischer R, Pakbaz Z, Onyekwere O, Porte, J, Swerdlow P, et al. Satisfaction and convenience of chelation therapy in patients with sickle cell disease (SCD): Comparison between deferasirox (Exjade ${ }^{\circledR}$, ICL670) and deferoxamine (DFO). Blood 2005;106(11 Pt 1). Abstract no.: 2334]

Vichinsky 2008 \{published data only\}

Vichinsky E, Pakbaz Z, Onyekwere O, Porter J, Swerdlow P, Coates T, et al. Patient-reported outcomes of deferasirox
(Exjade, ICL670) versus deferoxamine in sickle cell disease patients with transfusional hemosiderosis. Substudy of a randomized open-label phase II trial. Acta Haematologica 2008;119(3):133-41.

\section{Waheed 2014 \{published data only\}}

Waheed N, Ali S, Butt MA. Comparison of deferiprone and deferrioxamine for the treatment of transfusional iron overload in children with beta thalassemia major. Journal of Ayub Medical College, Abbottabad: JAMC 2014;26(3): 297-300.

\section{Walsh 2014 \{published data only\}}

Walsh KE, Cutrona SL, Kavanagh PL, Crosby LE, Malone $\mathrm{C}$, Lobner K, et al. Medication adherence among pediatric patients with sickle cell disease: a systematic review. Pediatrics 2014;134(6):1175-83.

Yarali 2006 \{published data only\}

Yarali N, Fisgin T, Duru F, Kara A, Ecin N, Fitoz S, Erden I. Subcutaneous bolus injection of deferoxamine is an alternative method to subcutaneous continuous infusion. Journal of Pediatric Hematology/Oncology 2006;28(1):11-6.

\section{References to studies awaiting assessment}

\section{Antmen 2013 \{published data only\}}

Antmen B, Organ K, Sasmaz I, Berktas M, Kilinc Y. A cohort study to assess the contribution of patient compliance program on persistence to deferasirox in patients with chronic iron overload in turkey (ex-pat program). Haematologica/the Hematology Journal 2013; Vol. 98: $713-4$.

\section{NCT00004982 \{published data only\}} NCT00004982. Combination iron chelation therapy. https://clinicaltrials.gov/ct2/show/NCT00004982 (accessed 18 April 2018).

\section{References to ongoing studies}

\section{EudraCT 2012-000353-31 \{published data only\}} Consorzio per le Valutazioni Biologiche e Farmacologiche. Multicentre, randomised, open label, non-inferiority active-controlled trial to evaluate the efficacy and safety of deferiprone compared to deferasirox in paediatric patients aged from 1 month to less than 18 years of age affected by transfusion-dependent haemoglobinopathies. https:// www.clinicaltrialsregister.eu/ctr-search/trial/2012-00035331/IT (EU Clinical Trials Register) (accessed 18 April 2018).

\section{IRCT2015101218603N2 \{published data only\}}

Amirmoezi F. To assess compliance, efficacy and satisfaction with two different formulation of deferasirox in patients with transfusion-dependent beta-thalassemia. http:// en.irct.ir/trial/16826 (Iranian Registry of Clinical Trails) (accessed 18 April 2018).

Madderom 2016 \{published data only\} Cnossen MH. A randomized trial evaluaTing the Effects of group medical AppointMents on self-efficacy and adherence in Sickle Cell Disease (TEAM study). - TEAM study 
[Effecten van GroepsconsulTen op zElfmAnageMent en therapietrouw in sikkelcelziekte (TEAM studie)]. http:// www.trialregister.nl/trialreg/admin/rctview.asp? TC $=4750$ (Erasmus Medical Center, Sophia Children's Hospital) (accessed 18 April 2018), issue NTR4750.

* Madderom MJ, Heijdra J, Utens EM, Polinder S, Rijneveld AW, Cnossen MH. A randomized controlled trial studying the effectiveness of group medical appointments on self-efficacy and adherence in sickle cell disease (TEAM study): study protocol. BMC Hematology 2016;16(21):6.

\section{NCT02173951 \{published data only\}}

NCT02173951. An algorithm to start iron chelation in minimally transfused young beta-thalassemia major patients. https://clinicaltrials.gov/ct2/show/NCT02173951 (accessed 18 April 2018):ClinicalTrials.gov.

\section{NCT02435212 \{published data only\}}

NCT02435212. Study to evaluate treatment compliance, efficacy and safety of an improved deferasirox formulation (granules) in pediatric patients $(2-<18$ years old) with iron overload. https://clinicaltrials.gov/ct2/ show/NCT02435212 (accessed 18 April 2018). Sponsors: Novartis Pharmaceuticals|NovartisOther IDs: CICL670F2202|2013-004739-55]

\section{Additional references}

\section{Abetz 2006}

Abetz l, Baladi JF, Jones P, Rafail D. The impact of iron overload and its treatment on quality of life: results from a literature review. Health and Quality of Life Outcomes 2006; 4:73.

\section{American Pharmacists Association 2008}

American Pharmacists Association, National Association of Chain Drug Stores Foundation. Medication therapy management in community pharmacy practice: core elements of an MTM service (version 2.0). American Pharmacists Association 2008:24.

\section{APPG 2009}

All-Party Parliamentary Group on Sickle Cell and Thalassaemia (APPG). Sickle cell disease and thalassaemia: A health check. http://ukts.org/pdfs/awareness/appg.pdf (accessed 20 June 2016).

\section{Aydinok 2014}

Aydinok Y, Kattamis A, Viprakasit V. Current approach to iron chelation in children. British Journal of Haematology 2014;165(6):745-55.

\section{Costello 2004}

Costello I, Wong ICK, Nunn AJ. A literature review to identify interventions to improve the use of medicines in children. Child: Care, Health and Development 2004;30(6): 647-65.

\section{Covidence [Computer program]}

Veritas Health Innovation. Covidence systematic review software. Melbourne, Australia: Veritas Health Innovation, 2017.

\section{Deeks 2011}

Deeks JJ, Higgins JPT, Altman DG on behalf of the Cochrane Statistical Methods Group, editor(s). Chapter 9: Analysing data and undertaking meta-analysis. In: Higgins JPT, Green S editor(s). Cochrane Handbook for Systematic Reviews of Interventions Version 5.1.0 (updated March 2011). The Cochrane Collaboration, 2011. Available from www.cochrane-handbook.org.

\section{EPOC 2015}

Effective Practice, Organisation of Care (EPOC). EPOC Resources for review authors. http://epoc.cochrane.org/ epoc-specific-resources-review-authors (accessed 21 September 2017).

\section{Fisher 2013}

Fisher SA, Brunskill SD, Doree C, Gooding S, Chowdbury O, Roberts DJ. Desferrioxamine mesylate for managing transfusional iron overload in people with transfusion-dependent thalassaemia. Cochrane Database of Systematic Reviews 2013, Issue 8. DOI: 10.1002/ 14651858.CD004450.pub3

\section{Gravitz 2014}

Gravitz L, Pincock S. Sickle-cell disease. Nature 2014;515: S1.

\section{Grosse 2011}

Grosse SD, Odame I, Atrash HK, Amendah DD, Piel FB, Williams TN. Sickle cell disease in Africa. A neglected cause of early childhood mortality. American Journal of Preventive Medicine 2011;41(6 S4):S298-S405.

\section{Haywood 2009}

Haywood, C, Beach M, Lanzkron S, Strouse, J, Wilson, $\mathrm{R}$, Park, $\mathrm{H}$, et al. A systematic review of barriers and interventions to improve appropriate use of therapies for sickle cell disease. Journal of the National Medical Association 2009;10(10):1022-33.

\section{Higgins 2011a}

Higgins JPT, Green S, editor(s). Cochrane Handbook for Systematic Reviews of Interventions Version 5.1.0 [updated March 2011]. The Cochrane Collaboration 2011. Available from www.cochrane-handbook.org.

\section{Higgins 2011b}

Higgins JPT, Deeks JJ, editor(s). Chapter 7: Selecting studies and collecting data. In: Higgins JPT, Green S, editor(s). Cochrane Handbook for Systematic Reviews of Interventions Version 5.1.0 [updated March 2011]. The Cochrane Collaboration, 2011. Available from www.cochrane-handbook.org.

\section{Higgins 2011c}

Higgins JPT, Altman DG, Sterne JAC on behalf of the Cochrane Statistical Methods Group and the Cochrane Bias Methods Group, editor(s). Chapter 8: Assessing risk of bias in included studies. In: Higgins JPT, Green S, editor(s). Cochrane Handbook for Systematic Reviews of Interventions. Version 5.1.0 [updated March 2011]. The Cochrane Collaboration, 2011. Available from www.cochrane-handbook.org. 


\section{Higgins 2011d}

Higgins JPT, Deeks JJ, Altman DG on behalf of the Cochrane Statistical Methods Group, editor (s). Chapter 16: Special topics in statistics. In: Higgins JPT, Green $S$, editor(s). Cochrane Handbook of Systematic Reviews of Interventions. Version 5.1.0 [updated March 2011]. The Cochrane Collaboration, 2011. Available from www.cochrane-handbook.org.

\section{Modell 2008}

Modell B, Darlison M. Global epidemiology of haemoglobin disorders and derived service indicators. Bulletin of the World Health Organization 2008;86(6):480-7.

\section{NCCMH 2010}

National Collaborating Centre for Mental Health. Depression in adults with a chronic physical health problem: treatment and management. Clinical guideline [CG91]. www.nice.org.uk/guidance/cg91 (accessed 16 April 2018). [ISBN:: 13: 978-1-904671-86-2]

\section{NCCPC 2009}

The National Collaborating Centre for Primary Care. Medicines adherence: involving patients in decisions about prescribed medicines and supporting adherence. NICE Guideline CG76. https://www.nice.org.uk/guidance/cg76 (accessed 20 June 2016).

\section{NICE 2009}

National Institute for Health and Care Excellence.

Depression in adults with chronic physical health problem: recognition and management. NICE guideline CG91. www.nice.org.uk/guidance/cg91 (accessed 20 June 2016).

\section{NICE 2010}

NICE National Institute for health and Care Excellence. Sickle cell disease. http://cks.nice.org.uk/sickle-celldisease\# !backgroundsub:3 (accessed 20 June 2016).

Payne 2008

Payne KA, Rofail D, Baladi JF, Viala M, Abetz L, Desrosiers MP, et al. Iron chelation therapy: clinical effectiveness, economic burden and quality of life in patients with iron overload. Advances in Therapy 2008;25(8):725-42.

Piel 2012

Piel FB, Patil AP, Howes RE, Nyangiri OA, Gething PW, Dewi M, et al. Global epidemiology of sickle haemoglobin in neonates: a contemporary geostatistical model-based map and population estimates. Lancet 2012;381(9861):142-51.

\section{Piel 2014}

Piel, FB, Weatherall DJ. The $\alpha$-thalassemias. New England Journal of Medicine 2014;371(20):1908-16.

\section{Pleasants 2014}

Pleasants S. Epidemiology: a moving target. Nature 2014; 515:S2-3

Rees 2010

Rees DC, Williams TN, Gladwin MT. Sickle-cell disease. Lancet 2010;376(9757):2018-31.

\section{Reeves 2011}

Reeves BC, Deeks JJ, Higgins JPT, Wells GA on behalf of the Cochrane Non-Randomised Studies Methods Group.
Chapter 13: Including non-randomized studies. In: Higgins JPT, Green S, editor(s). Cochrane Handbook for Systematic Reviews of Interventions Version 5.1.0 [updated March 2011]. The Cochrane Collaboration, 2011. Available from www.cochrane-handbook.org.

\section{RevMan 2014 [Computer program]}

The Nordic Cochrane Centre, The Cochrane Collaboration. Review Manager (RevMan). Version 5.3. Copenhagen: The Nordic Cochrane Centre, The Cochrane Collaboration, 2014.

\section{Rofail 2010}

Rofail D, Viala M, Gater A, Abetz-Webb L, Baladi JF, Cappellini MD. An instrument assessing satisfaction with iron chelation therapy: Psychometric testing from an openlabel clinical trial. Advances in Therapy 2010;27(8):533-46.

\section{Rund 2005}

Rund D, Rachmilewitz E. $\beta$-Thalassemia. New England Journal of Medicine 2005;353(11):1135-46.

\section{Ryan 2014}

Ryan R, Santesso N, Lowe D, Hill S, Grimshaw J, Prictor M, Kaufman C, Cowie G, Taylor M. Interventions to improve safe and effective medicines use by consumers: an overview of systematic reviews. Cochrane Database of Systematic Reviews 2014, Issue 4. DOI: 10.1002/ 14651858.CD007768.pub3

\section{Schünemann 2011a}

Schünemann HJ, Oxman AD, Higgins JPT, Vist GE, Glasziou P, Guyatt GH on behalf of the Cochrane Applicability and Recommendations Methods Group and the Cochrane Statistical Methods Group. Chapter 11: Presenting results and 'Summary of findings' tables. In: Higgins JPT, Green S, editor(s). Cochrane Handbook for Systematic Reviews of Interventions Version 5.1.0 [updated March 2011]. The Cochrane Collaboration, 2011. Available from www.cochrane-handbook.org.

\section{Sterne 2011}

Sterne JAC, Egger M, Moher D on behalf of the Cochrane Bias Methods Group, editor(s). Chapter 10: Addressing reporting biases. In: Higgins JPT, Green S, editor (s). Cochrane Handbook for Systematic Reviews of Interventions Version 5.1.0 [updated March 2011]. The Cochrane Collaboration, 2011. Available from www.cochrane-handbook.org.

\section{Sterne 2016}

Sterne JAC, Higgins JPT, Reeves BC on behalf of the development group for ROBINS-I. ROBINS-I: a tool for assessing Risk Of Bias In Non-randomized Studies of Interventions, Version 7 March 2016. www.riskofbias.info (accessed 19 April 2016).

\section{Telfer 2006}

Telfer P, Coen PG, Christou S, Hadjigavriel M, Kolnakou A, Pangalou E, et al. Survival of medically treated thalassemia patients in Cyprus. Trends and risk factors over the period 1980-2004. Haematologica 2006;91(9):1187-92. 


\section{Thomas 2013}

Thomas, V, Rawle, H, Abedian, M, Ferguson, A. Implementing the NICE clinical guideline 91 ('Depression in adults with a chronic physical health problem') in a haematology department. Clinical Psychology Forum 2013; 246:41-45.

Trachtenberg 2012

Trachtenberg FL, Mednick L, Kwiatkowski JL, Neufeld EJ, Haines D, Pakbaz Z, et al. Beliefs about chelation among thalassemia patients. Health and Quality of Life Outcomes 2012;10:148

Trachtenberg 2014

Trachtenberg, F, Gerstenberger, E, Xu, Y Mednick, L, Sobota, A, Ware, H, et al. Relationship among chelator adherence, change in chelators, and quality of life in Thalassemia. Quality of Life Research 2014;23:2277-88.

UK Thalassaemia Society 2008

United Kingdom Thalassaemia Society. Standards for the clinical care of children and adults with thalassaemia in the UK. UK Thalassaemia Society 2008; Vol. 2nd edition:120.

\section{Vekeman 2016}

Vekeman F, Sasane M, Cheng WY, Ramanakumar AV, Fortier J, Qiu Y, et al. Adherence to iron chelation therapy and associated healthcare resource utilization and costs in
Medicaid patients with sickle cell disease and thalassemia. Journal of Medical Economics 2016;19(3):292-303.

Wertheimer 2003

Wertheimer A, Santella T. Medication compliance research. Journal of Applied Research in Clinical and Experimental Therapeutics 2003;3(3):254-61.

\section{WHO 2003}

World Health Organization. Adherence to long-term therapies: Evidence for action. WHO 2003:194.

\section{Yawn 2014}

Yawn BP, Buchanan GR, Afenyi-Annan AN, Ballas SK, Hassell KL, James AH, et al. Management of sickle cell disease:summary of the 2014 evidence-based report by expert panel members. JAMA 2014;312(10):1033-48.

\section{References to other published versions of this review}

\section{Fortin 2016}

Fortin PM, Madgwick KV, Trivella M, Hopewell S, Doree C, Estcourt LJ. Interventions for improving adherence to iron chelation therapy in people with sickle cell disease or thalassaemia. Cochrane Database of Systematic Reviews 2016, Issue 9. DOI: 10.1002/14651858.CD012349

* Indicates the major publication for the study 


\title{
CHARACTERISTICS OF STUDIES
}

\section{Characteristics of included studies [ordered by study ID]}

\author{
Aydinok 2007
}

Methods

Participants
Study design: single-centre RCT

Study grouping: parallel group

Study duration: treatment duration 12 months; follow-up: not stated

\section{Baseline characteristics}

DFP, DFO

- Total \# of participants: 12 randomised; 8 analysed

- Age mean (SD): 16.6 (4.8) years, range 9 to 23 years

- Sex: not reported

- Ethnicity: not reported

- Thalassaemia genotype $\mathrm{N}(\%): 100 \% \beta$-thalassaemia

- Baseline ferritin levels (ng/mL) mean (SD): 4453 (2858)

- Previous iron chelation: not reported

- Duration of any iron chelation: not reported

- LIC (mg/g) mean (SD): 27.0 (13.4)

- Splenectomy n (\%): not reported

- QoL (mean (SD)): not reported

- Hb, g/L mean (SD): 89 (5)

DFP

- Total \# of participants: 12

- Age mean (SD): 15.9 (4.2) years

- Sex: not reported

- Ethnicity: not reported

- Thalassaemia genotype $\mathrm{N}(\%): 100 \% \beta$-thalassaemia

- Baseline ferritin levels (ng/mL): 4070 (3223)

- Previous iron chelation: not reported

- Duration of any iron chelation: not reported

- LIC (mg/g): 30.7 (10.6)

- Splenectomy n (\%): not reported

- QoL (mean (SD)): not reported

- $\mathrm{Hb}, \mathrm{g} / \mathrm{L}$ mean (SD): 89 (5), range 9 to 23 years

Inclusion criteria: iron-overloaded people with thalassaemia at least 4 years old

Exclusion criteria: lack of compliance, known toxicity or intolerance preventing therapy with DFO and DFP, neutropenia (neutrophils $<1.5 \times 10^{9} / \mathrm{L}$ ), thrombocytopenia (platelets $<100 \times 10^{9} / \mathrm{L}$ ), renal, hepatic or decompensated heart failure, active viral illness being treated with interferon- $\alpha /$ ribavirin, repeated Yersinia infections, HIV-positivity, pregnancy or nursing, and patients of reproductive age not taking adequate contraceptive precautions

Interventions

Treatment arm: DFO $(50 \mathrm{mg} / \mathrm{kg} /$ day subcutaneously twice weekly (mean (SD) dose: $43.8(2.8) \mathrm{mg} / \mathrm{kg})$ ) combined with DFP $(75 \mathrm{mg} / \mathrm{kg} /$ day, daily (mean (SD) dose: 78.2 (1.4) $\mathrm{mg} / \mathrm{kg} /$ day))

Comparator arm: DFP $(75 \mathrm{mg} / \mathrm{kg} /$ day, daily (mean (SD) dose: $78.2(2.6) \mathrm{mg} / \mathrm{kg} /$ day)) 
Outcomes
Adherence: compliance was assessed by drug accounting at each visit (by counting the returned empty blisters of DFP and used vials of DFO) as well as by a trial-specific questionnaire completed by the participants and/or their legal representative/guardian at quarterly intervals

The same questionnaire also served for the assessment of tolerance to treatment and QoL

Trial-reported outcomes

1. Changes in LIC and SF (primary outcome)

2. Total iron excretion

3. Urinary iron excretion

4. Iron balance

5. Cardiac function (Echo)

6. Toxicity

7. Assessment of tolerance to treatment and QoL

Identification

Source of funding: none stated although the drugs were supplied by Lipomed AG, Switzerland

Notes

All participants had prior exposure to DFO (dose, schedule and duration were not reported) and all had a washout period of 2 weeks with no iron chelation before initiating trial treatment

Sample-size calculation not reported

Country: Turkey

Risk of bias

\begin{tabular}{ll}
\hline Bias $\quad$ Authors' judgement \\
\hline
\end{tabular}

Random sequence generation (selection Low risk bias)

\section{Support for judgement}

"The randomization sequence was generated by the Department of Mathematical Statistics at the University of Berne, Switzerland according to local policy". Following central registration of a subject by the investigator, the trial co-ordinator assigned the intervention according to the randomisation sequence

The trial report states that the intervention was assigned according to the randomisation sequence "without concealing the sequence prior to allocation"

The authors did not report any information as to whether participants, personnel were blinded to treatment allocation but one treatment subcutaneous and other oral so difficult to blind
Blinding of participants and personnel High risk (performance bias)

All outcomes except mortality or other objective outcomes
High risk 
Aydinok 2007 (Continued)

Blinding of outcome assessment (detection Unclear risk bias)

All outcomes except mortality

Incomplete outcome data (attrition bias) High risk

All outcomes
The authors did not report any information as to whether outcome assessors were blinded to treatment allocation

There was an imbalance in missing data across the treatment arms. 4 participants from the comparator group (DFO) were not included in the outcome analysis: 2 withdrew consent due to refusal to take DFO; 1 died from arrhythmia induced congestive heart failure at start of trial; and 1 developed agranulocytosis at week 14

\begin{tabular}{|c|c|c|}
\hline Selective reporting (reporting bias) & Low risk & All outcomes were reported \\
\hline Other bias & Unclear risk & $\begin{array}{l}\text { There is an imbalance in baseline LIC and } \\
\text { Ferritin between groups }\end{array}$ \\
\hline
\end{tabular}

Badawy 2010

Methods

Study design: RCT

Study grouping: parallel group

Length of trial or follow-up not stated. Not stated if open label; but no mention of blinding and DFO is infusion versus tablet

Participants

\section{Baseline characteristics \\ DFP, DFO}

- Total \# of participants: 50

- Age: $\geq 8$ years

- Sex: not reported

- Ethnicity: not reported

- Thalassaemia genotype N (\%): $100 \% \beta$-thalassaemia

- Baseline ferritin levels $(\mathrm{ng} / \mathrm{mL})$ : not reported

- Previous iron chelation: DFO

- Duration of any iron chelation: not reported

- LIC (mg/g): not reported

- Splenectomy n (\%): not reported

- QoL (mean (SD)): not reported

- Hb, g/L: not reported

DFP

- Total \# of participants: 50

- Age: $\geq 8$ years

- Sex: not reported

- Ethnicity: not reported

- Thalassaemia genotype $\mathrm{N}(\%): \beta$-thalassaemia

- Baseline ferritin levels (ng/mL): not reported

- Previous iron chelation: DFO 
Badawy 2010 (Continued)

- Duration of any iron chelation: not reported

- Liver iron concentration LIC (mg/g): not reported

- Splenectomy n (\%): not reported

- QoL (mean (SD)): not reported

- Hb, g/L: not reported

DFO

- Total \# of participants: 50

- Age: greater or equal to 8 years

- Thalassaemia genotype N (\%): $100 \% \beta$-thalassaemia

- Baseline ferritin levels $(\mathrm{ng} / \mathrm{mL})$ : not reported

- Previous iron chelation: DFO

- Duration of any iron chelation: not reported

- LIC (mg/g): not reported

- Splenectomy n (\%): not reported

- QoL (mean (SD)): not reported

- $\mathrm{Hb}, \mathrm{g} / \mathrm{L}$ : not reported

Inclusion criteria: 8 years, RBC transfusion every 3 to 4 weeks, on DFO prior to study as single therapy

Exclusion criteria: not stated

Participants PRBCs $/ 3$ - 4 weeks to maintain $\mathrm{Hb}>9 \mathrm{~g} / \mathrm{dL}$

Interventions

\section{DFP, DFO}

- Medication intervention: daily DFP, DFO twice-weekly DFO (40 mg/kg/day);

Deferipron e $(75 \mathrm{mg} / \mathrm{kg} /$ day $)$.

DFP

- Medication intervention: daily DFP Deferipron e $(75 \mathrm{mg} / \mathrm{kg} /$ day $)$. DFO

- Medication intervention: DFO 5 days/week DFO (40 mg/kg/day)

Outcomes

Adherence to iron chelation therapy rates

Questionnaire on chelation therapy, reasons for non-compliance, side effects, life activities, transfusion regimen

Trial-reported outcomes

1. CBC monthly

2. SF levels

3. liver and kidney functions

4. blood glucose level

5. serum calcium and phosphorus/3 months and T3, T4,TSH, LH, FSH

6. echocardiography

7. bone density

8. auditory and visual examination twice

Identification

Sponsorship source: Zagazig University Hospital, Zagazig

Country: Egypt

Setting: University Hospital

Comments: Abstract Poster 124

Authors name: Sherif Badawy

Institution: Ann Robert H. Lurie Children's Hospital of Chicago

Email: sbadawy@luriechildrens.org

Interventions for improving adherence to iron chelation therapy in people with sickle cell disease or thalassaemia (Review) 
Address: Ann Robert H. Lurie Children's Hospital of Chicago Northwestern University Feinberg School of Medicine225 East Chicago Avenue, Box 30, Chicago, Illinois 60611 2605

\begin{tabular}{ll}
\hline Notes & $\begin{array}{l}\text { Contacted author and study data not available at this time. Sample-size calculation not } \\
\text { reported }\end{array}$
\end{tabular}

\section{Risk of bias}

\begin{tabular}{|c|c|c|}
\hline Bias & Authors' judgement & Support for judgement \\
\hline $\begin{array}{l}\text { Random sequence generation (selection } \\
\text { bias) }\end{array}$ & Unclear risk & $\begin{array}{l}\text { Judgement comment: no description of se- } \\
\text { quence generation }\end{array}$ \\
\hline Allocation concealment (selection bias) & Unclear risk & $\begin{array}{l}\text { Judgement comment: no description of al- } \\
\text { location concealment }\end{array}$ \\
\hline $\begin{array}{l}\text { Blinding of participants and personnel } \\
\text { (performance bias) } \\
\text { All outcomes except mortality or other ob- } \\
\text { jective outcomes }\end{array}$ & High risk & $\begin{array}{l}\text { Judgement comment no description, but } \\
\text { one drug is subcutaneous injection (DFO) } \\
\text {. Open label }\end{array}$ \\
\hline $\begin{array}{l}\text { Blinding of outcome assessment (detection } \\
\text { bias) } \\
\text { All outcomes except mortality }\end{array}$ & Unclear risk & $\begin{array}{l}\text { Judgement comment: no description of } \\
\text { blinding of assessors }\end{array}$ \\
\hline $\begin{array}{l}\text { Incomplete outcome data (attrition bias) } \\
\text { All outcomes }\end{array}$ & High risk & $\begin{array}{l}\text { Judgement comment: no data on number } \\
\text { of participants who completed the study } \\
\text { and how many in each group experienced } \\
\text { complications. Lack of detail on number of } \\
\text { compliant or non-compliant participants }\end{array}$ \\
\hline Selective reporting (reporting bias) & High risk & $\begin{array}{l}\text { Judgement comment: not clear which } \\
\text { groups and how many experienced adverse } \\
\text { events. No data reported on SF or other } \\
\text { outcomes }\end{array}$ \\
\hline Other bias & Unclear risk & $\begin{array}{l}\text { Judgement comment: results of the trial } \\
\text { were not published in detail and no data } \\
\text { available when authors were contacted }\end{array}$ \\
\hline
\end{tabular}

Bahnasawy 2017

Methods
Study design: single-centre RCT

Study grouping: parallel group

Study duration: 6 months 
Participants

\section{Baseline characteristics}

Comprehensive medication management

- Total \# of participants: 24

- Age (mean (SD)): 12 (2.7)

- Sex N (\%): F: 15 (62.5); M: 9 (37.5)

- Ethnicity: not reported

- Thalassaemia genotype (\%): $\beta$-thalassaemia major $100 \%$

- Baseline ferritin levels (ng/mL) (mean (SD)): 3949 (1864)

- Previous iron chelation: N/A

- Duration of any iron chelation: N/A

- LIC $(\mathrm{mg} / \mathrm{g})$ : not stated

- Splenectomy n (\%): 6 (25.9)

- QoL PedsQL median (IQR): 55.16 (43.42 - 63.75)

- Hb, g/L: not stated

Standard care (as defined in the trial)

- Total \# of participants: 24

- Age (mean (SD)): 13 (2.8)

- Sex N (\%): F: 15 (62.5); M: 9 (37.5)

- Ethnicity: not reported

- Thalassaemia genotype (\%: $\beta$-thalassaemia major $100 \%$

- Baseline ferritin levels (ng/mL) (mean (SD)): 3871 (1881)

- Previous iron chelation: N/A

- Duration of any iron chelation: N/A

- LIC (mg/g): not stated

- Splenectomy n (\%): 9 (37.5)

- QoL PedsQL median (IQR): 49.12(38.13 - 56.95)

- Hb, g/L: not stated

Inclusion criteria: transfusion-dependent children with $\beta$-thalassaemia major aged 8 to 18 years with SF level of more than $1000 \mu \mathrm{g} / \mathrm{L}$

Exclusion criteria: people with cognitive impairment

\section{Comprehensive medication management}

- interview with participants at each visit, drug-related problems identified, care plan introduced / monitored to include dosage modification, education. Follow-up compliance via regular phone calls

\section{Standard care (as defined in the trial)}

- all participants presented to the clinic regularly every $2-4$ weeks according to the need for receiving blood transfusion, blood samples were drawn for CBC assessment. Physical examination was done by physician including assessment of hepatomegaly, splenomegaly and any health-related problems

Adherence to iron chelation therapy rates "DRP identification: The clinical pharmacist analysed the collected data to detect whether any DRPs existed and allocated them to one of the seven categories as classified by Cipolle et al. [18]: unnecessary drug therapy, need for additional drug therapy, ineffective drug product, dosage too low, adverse drug reaction, dosage too high, noncompliance"

\section{Trial-reported outcomes}

1. SF levels were measured at baseline, 3 months and after 6 months 
2. CBC with WBC differential was assessed at every visit, and SCr and ALT were measured routinely for all the participants every 3 months

3. Health-related QoL was assessed at baseline and at the end of the trial (after 6 months) using PedsQL ${ }^{\text {TM }} 4.0$ Generic Core Scale questionnaire. PedsQL is a 23-item multidimensional model with 4 domains for paediatric health-related QoL measurement: physical functioning ( 8 items), emotional functioning ( 5 items), social functioning (5 items) and school functioning (5 items) (19)

Identification

Sponsorship source: not stated

Country: Egypt

Setting: Hematology clinic

Authors name: Lamia El Wakeel

Institution: Pediatric Hematology Clinic, Children's Hospital, Ain Shams University,

Email: lamywak@yahoo.com

Address: Lamia El Wakeel, Pediatric Hematology Clinic, Children's Hospital, AinShams

University, 4, Street 292 New Maadi, Cairo, Egypt

Notes

Sample-size calculation not reported.

Drug-related outcomes do not have any comparable data reported. Only outcomes with comparable data reported are SF levels and health-related QoL

\section{Risk of bias}

\section{Bias} bias)
Random sequence generation (selection Unclear risk

Authors' judgement

\section{Support for judgement}

Quote: "The study was a prospective, randomized, controlled study. It was conducted on pediatric BTM patients admitted to the Pediatric Hematology Clinic," Stratified randomization was used considering the iron chelation therapy as the stratification factor

Judgement comment: no description of how randomisation was done or by whom

Allocation concealment (selection bias) Unclear risk

Blinding of participants and personnel High risk (performance bias)

All outcomes except mortality or other objective outcomes dard medical care by a physician while the intervention group received standard medical care plus clinical pharmacist-provided services

Judgement comment: no description of how participants were allocated to the pharmacist intervention or standard care

Judgement comment: not possible to blind a pharmacist intervention versus no pharmacist intervention
The control group $(\mathrm{n}=24)$ received stan- 
Blinding of outcome assessment (detection High risk bias)
All outcomes except mortality

Judgement comment: no indication that outcome assessors where different from pharmacists who implemented the intervention. Also most outcomes were reported only in the intervention group except for ferritin levels and health-related QoL

Judgement comment: all drug-related outcomes were only reported in the intervention group including adherence - no comparative data available. Multiple interventions in small number of participants

Selective reporting (reporting bias) High risk

Judgement comment: drug-related outcomes reported only in intervention group. No comparative data. The participants within the intervention arm seem to have complex and multiple changes. Difficult to tease out the actual intervention that effected a change

Other bias Unclear risk

Judgement comment: small sample size and only report intervention group

Calvaruso 2015

Methods

Participants

\section{Study design: RCT}

Study grouping: parallel group

This trial was designed as a 5-year, multicentre, randomised, open-label trial with blinded data management and data analyses to evaluate whether the DFP treatment is superior to the DFO treatment

Follow-up after trial. An additional 5 years of follow-up after the end of the trial was planned to collect data on the survival, cause of death and chelation treatment of this cohort of participants. During this period, the participants were allowed to change their chelation treatment

\section{Baseline characteristics}

DFP

- Total \# of participants: 47

- Age: mean (SD): 41.3 (14.8)

- Sex n (\%): F: 24 (50)

- Ethnicity: not reported

- Thalassaemia genotype (\%): thalassaemia Intermedia $100 \%$

- Baseline ferritin levels (ng/mL) median (IQR): 1221 (743)

- Age at initiation of DFO years: mean (SD): 29.9 (16.8)

- LIC (mg/g/dw) median (IQR): 3800 (2800)

- Splenectomy n (\%): 42 (89.3)

- QoL: mean (SD): not reported 
- Total \# of participants: 41

- Age: mean (SD): 41.2 (14.3)

- Sex n (\%): F: 23 (51.1)

- Ethnicity: not reported

- Thalassaemia genotype (\%): thalassemia intermedia $100 \%$

- Baseline ferritin levels (ng/mL) (median (IQR)): 1,122 (910)

- Age at initiation of DFO years: mean (SD): 29.6 (17.4)

- LIC (mg/g/dw) median (IQR): 3800 (4668)

- Splenectomy n (\%): 35 (77.7)

- QoL: mean (SD): not reported

- $\mathrm{Hb}, \mathrm{g} / \mathrm{L}$ mean (SD): 89 (12)

Inclusion criteria: people with thalassaemia intermedia (based on clinical and molecular criteria), SF between 800 and $3000 \mu \mathrm{g} / \mathrm{L}, 13$ years of age, consent from patient or parent or guardian (if 13 to 18 )

Exclusion criteria: known intolerance to treatment, platelet count $<100 \times 10^{9} / \mathrm{L}$, white cell count of $<3 \times 10^{9} / \mathrm{L}$, severe liver damage, sepsis or heart failure (or both)

Pretreatment: none of the participants in the DFP group and 8 in the DFO group withdrew from the trial. 1 participant in the DFP group and 3 in the DFO group changed their chelation therapy $(\mathrm{P}$ value $=0.357)$

If the participants were treated with a subcutaneous administration of DFO (30 - 50 mg/ $\mathrm{kg}$ per day, 8 - 12 hours for 5 days a week) before inclusion in the trial, a DFO washout was executed for 1 week before randomisation. The minimum number of participants required for each treatment group was calculated, assuming equal allocation under the hypothesis of equality between the 2 treatment groups at each point during the course. The recommended number of participants was 30

One participant in the DFP group and 3 in the DFO group changed their chelation therapy

- DFP (Apotex; Toronto, ON, Canada) administered at $75 \mathrm{mg} / \mathrm{kg} /$ day, divided into 3 oral daily doses for 7 days/week

\section{DFO}

- DFO (BiofuturaPharma, Omezia, Italy), administered by subcutaneous infusion (8 - 10 hours) at $50 \mathrm{mg} / \mathrm{kg}$ per day for 5 days/week

Treatment failure was defined as an increase in the SF level to greater than $1000 \mathrm{lg} / \mathrm{L}$ from baseline, confirmed by at least 2 consecutive determinations. Participants who failed were switched to the alternative treatment and followed until the end of the trial. The criteria for a dosage reduction to $50 \mathrm{mg} / \mathrm{kg}$ of DFP per day were arthralgia and nausea, and the criterion for a reduction to $30 \mathrm{mg} / \mathrm{kg}$ of DFO per day was a local reaction at the site of infusion. Both treatments were reduced if the ferritin levels for 2 consecutive determinations were less than $400 \mathrm{lg} / \mathrm{L}$. The treatment was resumed when the ferritin levels were greater than $700 \mathrm{lg} / \mathrm{L}$ for at least 2 determinations 
Calvaruso 2015 (Continued)

1. The primary endpoint was treatment effectiveness, evaluated as the mean change in the SF level over the 5-year period. This type of evaluation strengthened the power of the test for the sample-size calculation compared with the standard

2. The secondary endpoints were safety and survival analysis after 5 years

Identification

Sponsorship source: contract grant sponsor: Franco and Piera Cutino Foundation

Country: Italy (17 centres)

Setting: haematology and thalassaemia clinical centres at institutions

Recruitment: January 2001 to January 2006

Trial registration: NCT00733811

Authors name: Aurelio Maggio

Institution: Unita Operativa Complessa Ematologia II,

Email: md.amaggio@gmail.com

Address: U.O.C. Ematologia II, A.O.R. "Villa Sofia - V. Cervello", Palermo, Italy

\begin{tabular}{l|l} 
Sotes & Sample-size calculation reported for primar \\
Notes: 9 participants changed from DFP the & 5 to DFO \\
2 to none \\
1 to DFX \\
1 to \\
DFP-DFO \\
6 participants changed from DFO therapy \\
4 to DFP \\
1 to DFX \\
1 to DFP-DFO
\end{tabular}

Risk of bias

\begin{tabular}{ll|l}
\hline Bias & Authors' judgement & Support for judgement \\
\hline $\begin{array}{l}\text { Random sequence generation (selection } \\
\text { bias) }\end{array}$ & Low risk & $\begin{array}{l}\text { Quote: "The randomization sequence was } \\
\text { based on a computer- randomized list ar- } \\
\text { ranged in permuted blocks of 10 with a 1: } \\
\text { 1 ratio." }\end{array}$ \\
\hline
\end{tabular}

Allocation concealment (selection bias) Low risk

To ensure for allocation concealment, treatments were assigned by telephone contact from the coordinating centre. The sequence was concealed until the interventions were assigned. Randomization was performed for each consecutive patient after verification of the exclusion criteria

Blinding of participants and personnel High risk (performance bias)

All outcomes except mortality or other objective outcomes

Quote: “open-label trial”

Judgement comment: 1 of 2 arms was desferal pump infusers, participants would know. Participants on DFO attended for weekly blood tests

Interventions for improving adherence to iron chelation therapy in people with sickle cell disease or thalassaemia (Review) 
Calvaruso 2015 (Continued)

Blinding of outcome assessment (detection Low risk

bias)

Quote: "with blinded data management and data analysis"

All outcomes except mortality

Incomplete outcome data (attrition bias) Low risk

No loss to follow-up for 5-year trial

All outcomes

\begin{tabular}{|c|c|c|}
\hline Selective reporting (reporting bias) & Low risk & All outcomes reported \\
\hline Other bias & Unclear risk & $\begin{array}{l}\text { Unclear how participant variation relating } \\
\text { to SF levels may have had effect on results. } \\
\text { Although all outcomes were reported for } \\
\text { the } 5 \text { year trial in the } 5 \text { years of follow-up } \\
\text { only mortality was reported }\end{array}$ \\
\hline
\end{tabular}

El Beshlawy 2008

Methods

Study design: single-centre RCT

Study grouping: parallel group, follow-up for 54 weeks

Participants

\section{Baseline characteristics}

\section{DFP/DFO}

- Total \# of participants: 18

- Age (mean (SD): 11.0 (4.9)

- Sex: F: 10; M: 8

- Ethnicity: not reported

- Thalassaemia genotype $\mathrm{N}(\%)$ : $\beta$-thalassaemia major: $100 \%$

- Baseline ferritin levels (ug/mL) (mean (SD) (range)): 2865 (983) (1500 - 4800)

- Previous iron chelation: not reported

- LIC (mg/g) mean (SD) (range): 17.1 (9.1) (4.9 - 33.6) $\mathrm{N}=16$

- Splenectomy n (\%): 11 (61)

- QoL mean (SD): not reported

- $\mathrm{Hb}, \mathrm{g} / \mathrm{L}$ (mean (SD) (range): 68 (5) (55 - 75)

DFP

- Total \# of participants: $\mathrm{N}=18$

- Age (mean (SD) (range)): 10.8 (5.1) (5 - 26)

- Sex: F: 6; M: 12

- Ethnicity: not reported

- Thalassaemia genotype $\mathrm{N}(\%)$ : $\beta$-thalassaemia major: $100 \%$

- Baseline ferritin levels (ug/mL) (mean (SD) (range)): 2926 (1107) (1560 - 5000)

- Previous iron chelation: not reported

- $\mathrm{LIC}(\mathrm{mg} / \mathrm{g})$ (mean (SD) (range)): 15.8 (7.1) (2.3 - 29.3) $\mathrm{N}=17$

- Splenectomy n (\%): 9 (50)

- QoL mean (SD): not reported

- $\mathrm{Hb}, \mathrm{g} / \mathrm{L}$ mean (SD) (range): 69 (6) (58 - 80)

DFO

- Total \# of participants: $\mathrm{N}=20$

Interventions for improving adherence to iron chelation therapy in people with sickle cell disease or thalassaemia (Review) 
El Beshlawy 2008 (Continued)

- Age (mean (SD) (range)): 13.1 (5.9) (5.5 - 24)

- Sex: F: 9; M: 11

- Ethnicity: not reported

- Sickle cell genotype $\mathrm{N}(\%)$ - not applicable:

- Thalassaemia genotype $\mathrm{N}(\%)$ : $\beta$-thalassaemia major: $100 \%$

- Baseline ferritin levels (ug/mL) (mean (SD)(range)): 2838 (967) (1500 - 4300)

- Previous iron chelation: not reported

- LIC (mg/g) mean (SD) (range): 22.5 (10.1) $(6.0$ - 41.7) $\mathrm{N}=15$

- Splenectomy n (\%): 10 (50)

- QoL mean (SD): not reported

- $\mathrm{Hb}, \mathrm{g} / \mathrm{L}$ mean (SD) (range): 69 (5) (60 - 80)

Inclusion criteria: males or females with thalassaemia major attending the Hematology Clinic at Cairo University Children Hospital; participants had to be iron overloaded with transfusion dependency and older than 4 years of age

Exclusion criteria: known to have DFP or DFO toxicity; neutrophil count less than 1. $5 \times 10^{9} / \mathrm{L}$; platelet count less than $100 \times 10^{9} / \mathrm{L}$; renal or hepatic insufficiency; decompensated heart failure; without contraceptive precaution; pregnant or nursing

Interventions

DFP/DFO

- DFP + DFO (dose $60-83 \mathrm{mg} / \mathrm{kg} /$ day and DFO 23 to $50 \mathrm{mg} / \mathrm{kg}$ per dose) DFP 7 days and DFO over 8 hours 2 days/week)

DFP

- DFP only (dose 60 to $83 \mathrm{mg} / \mathrm{kg} /$ day) 7 days per week DFO

- DFO 23 to $50 \mathrm{mg} \mathrm{kg} /$ day monotherapy for 5 days/week

Outcomes

Adherence to iron chelation therapy rates

Compliance was assessed by performing a drug accounting at each patient visit by counting the returned empty blisters of DFP and used vials of DFO

Trial-reported outcomes

1. Incidence of chelation therapy-related SAEs (reported in AEs)

2. Iron overload defined by ferritin over $1000 \mu \mathrm{g} / \mathrm{L}$ and/or clinical symptoms and/or signs of iron overload and/or need for medically indicated additional or change in chelation therapy (mean ferritin levels extrapolated from graph - no SD provided)

3. Other AEs related to iron chelation (in this trial participants with an event are reported. 1 person could experience more than 1 event)

4. LIC mg/g dry weight (change from baseline (extrapolated from graph Least squares means / lower and upper value))

Identification

Sponsorship source

Country: Egypt

Setting: Hematology Clinic at Cairo University Children Hospital, Egypt

Comments: 2 authors from Lipomed (DFP): C. Manz : C. Tarabishi Clinical Research Development, Lipomed AG, Arlesheim, Switzerland

Authors name: A. El-Beshlawy

Institution: Faculty of Medicine, Cairo University,

Email: amalelbeshlawy@yahoo.com

Address: Faculty of Medicine, Cairo University, 32 Falaky Street, Bab El-Louk, Cairo, Egypt

Interventions for improving adherence to iron chelation therapy in people with sickle cell disease or thalassaemia (Review) 
El Beshlawy 2008 (Continued)

\begin{tabular}{|c|c|c|}
\hline Notes & \multicolumn{2}{|c|}{ Sample-size calculation reported } \\
\hline \multicolumn{3}{|l|}{ Risk of bias } \\
\hline Bias & Authors' judgement & Support for judgement \\
\hline $\begin{array}{l}\text { Random sequence generation (selection } \\
\text { bias) }\end{array}$ & Unclear risk & $\begin{array}{l}\text { Judgement comment: no description of } \\
\text { how randomisation was accomplished: The } \\
\text { participants were randomly assigned into } 1 \\
\text { of } 3 \text { treatment arms }\end{array}$ \\
\hline Allocation concealment (selection bias) & Unclear risk & $\begin{array}{l}\text { Judgement comment: no description of al- } \\
\text { location concealment }\end{array}$ \\
\hline $\begin{array}{l}\text { Blinding of participants and personnel } \\
\text { (performance bias) } \\
\text { All outcomes except mortality or other ob- } \\
\text { jective outcomes }\end{array}$ & High risk & $\begin{array}{l}\text { No mention of blinding - since DFO is an } \\
\text { injection and DFP is oral likely participants } \\
\text { and personnel not blinded }\end{array}$ \\
\hline $\begin{array}{l}\text { Blinding of outcome assessment (detection } \\
\text { bias) } \\
\text { All outcomes except mortality }\end{array}$ & Unclear risk & $\begin{array}{l}\text { Judgement comment: no blinding men- } \\
\text { tioned }\end{array}$ \\
\hline $\begin{array}{l}\text { Incomplete outcome data (attrition bias) } \\
\text { All outcomes }\end{array}$ & High risk & $\begin{array}{l}\text { Judgement comment: a total of } 10 \text { partici- } \\
\text { pants dropped out of the trial as a result of } \\
\text { several complications. Only } 56 \text { participants } \\
\text { completed } 54 \text { weeks of treatment. Evalua- } \\
\text { tion of LIC could not be done in another } 8 \\
\text { participants. Reports on per protocol par- } \\
\text { ticipants }\end{array}$ \\
\hline Selective reporting (reporting bias) & High risk & $\begin{array}{l}\text { Compliance not reported as number or per- } \\
\text { centage of participants compliant through- } \\
\text { out trial: "Four patients, all treated with } \\
\text { DFO-based regimen, were excluded from } \\
\text { the study due to lack of compliance. Com- } \\
\text { pliance was otherwise excellent during the } \\
\text { entire study. The majority of patients had } \\
\text { no problems with the intake and swallow- } \\
\text { ing of the DFP tablets. By contrast, } 80 \% \text { of } \\
\text { patients in the combination arm and } 76 \% \\
\text { of patients in the DFO monotherapy arm } \\
\text { complained about difficulties in the par- } \\
\text { enteral use of DFO or problems to insert a } \\
\text { needle", SF and LIC are partially reported } \\
\text { in charts and no actual numbers are pro- } \\
\text { vided in the text. Also the focus on UIE over } \\
\text { LIC and SF measures is misleading as DFP }\end{array}$ \\
\hline
\end{tabular}

Interventions for improving adherence to iron chelation therapy in people with sickle cell disease or thalassaemia (Review) 
El Beshlawy 2008 (Continued)

is known to have a higher UIE but this can be highly variable over multiple measurements. LIC is the gold standard and there was no difference in this outcome between groups

Other bias Unclear risk

There was a higher incidence of AEs in the combined group and the DFP group versus the DFO group

Elalfy 2015

Methods

Study design: RCT in 2 treatment centres

Study grouping: parallel group

Study duration: 1 year

Participants

Baseline characteristics

Group A: DFP/DFO

- Total \# of participants: 48

- Age: mean (SD): 15.25 (2.31)

- Sex: male n (\%): 30 (62.5)

- Ethnicity: not reported

- Thalassaemia genotype $\mathrm{N}(\%)$ : Not stated all participants appear to have $\beta$ thalassaemia major

- Baseline ferritin levels (ng/mL): mean (SD): 4379.07 (895.00); range 3632 - 6210

- Duration of any iron chelation (years): mean (SD): 8.71 (2.7)

- LIC (mg/g): mean (SD): 12.69 (2.23); range: 12.69 - 2.23

- Splenectomy n (\%): 21 (43.7)

- QoL mean (SD): 63.09 (5.77)

- $\mathrm{Hb}, \mathrm{g} / \mathrm{L}$ mean (SD): 81.1 ( 3.3$)$

- Mean geometric cardiac T2*(ms): mean (SD): 16.32 (1.82); range: 14.9 - 18.2

Group B: DFP/DFX

- Total \# of participants: 48

- Age: mean (SD): 14.05 (2.21)

- Sex: male n (\%): 32 (66.6)

- Ethnicity: not reported

- Thalassaemia genotype $\mathrm{N}(\%)$ : not stated all participants appear to have $\beta$ thalassaemia major

- Baseline ferritin levels (ng/mL) mean (SD): 4289.19 (866.21); range: 3451 - 7122

- Duration of any iron chelation (years): mean (SD): 8.95 (2.8)

- LIC (mg/g): mean (SD): 12.52 (2.28); range: 9.82 - 15.12

- Splenectomy n (\%): 20 (41.6)

- QoL mean (SD): 63.38 (5.98)

- $\mathrm{Hb}, \mathrm{g} / \mathrm{L}$ mean (SD): 79 (3.8)

- Mean geometric cardiac T2*(ms): mean (SD):16.59 (1.85); range: 15.7 - 18.9

Inclusion criteria: people with $\beta$-thalassaemia major aged $10-18$ years with severe iron overload defined as: ferritin $>2500 \mu \mathrm{g} / \mathrm{L}$ on maximum tolerated dose of a single iron chelator with up trend of ferritin over the last 12 months prior to the study. People

Interventions for improving adherence to iron chelation therapy in people with sickle cell disease or thalassaemia (Review) 
with LIC more than $7 \mathrm{mg} / \mathrm{g}$ by MRI R2* and mean cardiac T2* less than 20 and more than $6 \mathrm{~ms}$ calculated as geometric mean without clinical symptoms of cardiac dysfunction (shortness of breath at rest or exertion, orthopnoea, exercise intolerance, lower extremity oedema, arrhythmias). Adequacy of prior chelation defined as taking $75 \%$ of the calculated dose/month on maximum tolerated dose with upward ferritin trend

Exclusion criteria: past history of agranulocytosis, clinically significant GI or renal disease, clinical cardiac disease, or with LVEF $<50 \%$ on baseline echocardiography; evidence of active hepatitis or serum transaminases $>3$ times above ULN or renal impairment (serum creatinine > ULN) participation in a previous investigational drug study within the 30 days preceding screening, known allergy to DFX, DFP, and DFO

Pre-treatment: baseline difference in mean $\mathrm{Hb}$ (P 0.004)

Interventions

Outcomes

\section{DFP/DFO}

- DFP $75 \mathrm{mg} / \mathrm{kg} /$ day divided into 2 doses taken orally at 8 a.m. and 3 p.m. for 7 days (with 6 - 8 hours interval between the 2 doses) combined with DFO $40 \mathrm{mg} / \mathrm{kg}$ / day by subcutaneous infusion over 10 hours starting at 10 p.m. for 6 days/week

\section{DFP/DFX}

- DFP $75 \mathrm{mg} / \mathrm{kg} / \mathrm{day}$, divided into 2 doses taken orally at 8 a.m. and 3 p.m. combined with DFX30 mg/kg/day taken orally at 10 p.m. for 7 days/week

To achieve an acceptable treatment washout, chelation therapy was withdrawn for 2 weeks before randomisation, after verifying inclusion and exclusion criteria. The transfusion regimen aimed to maintain the participants pre-transfusion $\mathrm{Hb} \geq 80 \mathrm{~g} / \mathrm{L}$ by receiving approximately $15 \mathrm{~mL} / \mathrm{kg}$ packed RBCs every 3 - 4 weeks

\begin{tabular}{l} 
Adherence to iron chelation therapy rates \\
Compliance was evaluated by counting of returned tablets for the oral chelators and of \\
the vials for DFO. The percentage of actual dose that participant had taken in relation \\
to the total prescribed dose was calculated \\
Trial-reported outcomes \\
\begin{tabular}{l} 
1. \% change in SF (from baseline to the end of trial) \\
2. \% change in LIC (from baseline to the end of trial) \\
3. \% change in cardiac MRI (from baseline to the end of trial) \\
4. SAEs and AEs (safety assessment) \\
5. Compliance \\
6. Satisfaction \\
7. QoL \\
\hline Sponsorship source: Ain Shams University \\
Country: Egypt and Oman \\
Setting: Thalassemia treatment centres (Ain Shams University, Egypt and Sultan Qaboos \\
University Hospital, Oman) \\
Comments: Government Clinical Trial NCT01511848 \\
Authors name: Amira Abdel Moneam Adly, \\
Institution: Department of Pediatrics, Ain Shams University, Cairo, Egypt \\
Email: amiradiabetes@yahoo.com \\
Address: 6 A ElSheshini street, Shoubra, Soudia buildings, Cairo, Egypt
\end{tabular} \\
\hline
\end{tabular}

Interventions for improving adherence to iron chelation therapy in people with sickle cell disease or thalassaemia (Review) 
Elalfy 2015 (Continued)

\begin{tabular}{l} 
The chelation regimens in the last year prior to the trial were daily DFX (14 participants) \\
, daily DFP (29 participants), and DFP 4 days/week alternating with subcutaneous DFO \\
3 days/week ( 53 participants) \\
Sample-size calculation reported \\
Author contacted for additional info on SF 36 mean (SD) 6 months and end of trial \\
\hline
\end{tabular}

Risk of bias

\begin{tabular}{|c|c|c|}
\hline Bias & Authors' judgement & Support for judgement \\
\hline $\begin{array}{l}\text { Random sequence generation (selection } \\
\text { bias) }\end{array}$ & Low risk & $\begin{array}{l}\text { Quote: "The randomisation sequence was } \\
\text { based on a computer randomised list in per- } \\
\text { muted blocks of } 10 \text { with a } 1: 1 \text { ratio, gen- } \\
\text { erated at both University of Ain Shams and } \\
\text { Sultan Qaboos" }\end{array}$ \\
\hline
\end{tabular}

Allocation concealment (selection bias) Low risk

Quote: "To ensure no allocation bias, treatment group was assigned by telephone contact from the coordinating center in Ain Shams"

Blinding of participants and personnel High risk (performance bias)

All outcomes except mortality or other objective outcomes

Oral versus subcutaneous medication therefore participants would be aware which medication arm they had been randomised to

Blinding of outcome assessment (detection Low risk bias)

Quote: “open-label study with blinded data management and data analyses"

All outcomes except mortality

Incomplete outcome data (attrition bias) Low risk

All outcomes

Selective reporting (reporting bias)

Unclear risk
Judgement comment: treatment was started within the following $24 \mathrm{hr}$, and all the included participants continued till the end of study with no participants were lost follow-up

Judgement comment: provide only P values for patient satisfaction, satisfaction with ICT self-reported satisfaction and all 'significantly' higher in group B; no actual end of trial data provided (mean (SD)). All outcomes are reported

Other bias

Unclear risk
Judgement comment: it is not clear how the investigators would have known that infections, GI disorders or skin disorders were not related to the drug therapies 
Methods

Participants
Study Design: 2-arm parallel RCT conducted in Italy and Greece

Number of centres: multicentre (3 centres)

Duration of treatment: 12 month

Follow-up: not stated.

\section{DFP/DFO}

- Total \# of participants: randomised 30, analysed 29 (withdrawn after 2 days on trial before taking DFP)

- Age (mean (SD): 19.8 (6.1) years

- Sex: F: 13; M: 16

- Ethnicity: not reported

- Thalassaemia genotype $\mathrm{N}(\%)$ : $\beta$-thalassaemia major: $100 \%$

- Baseline ferritin levels (ug/mL) mean (SD): 2048 (685)

- Previous iron chelation: not reported

- LIC (mg/g) mean (SD) (range): 17.1 (9.1) (4.9 - 33.6) $\mathrm{N}=16$

- Splenectomy n (\%): 11 (61)

- QoL mean (SD): not reported

- $\mathrm{Hb}, \mathrm{g} / \mathrm{L}$ mean (SD) (range): 68 (5) (55 - 75)

\section{DFP/DFO}

- Total \# of participants: randomised 30, analysed 30

- Age (mean (SD)): 18.7 (4.8) years

- Sex: F: 18; M: 12

- Ethnicity: not reported

- Thalassaemia genotype $\mathrm{N}(\%)$ : $\beta$-thalassaemia major: $100 \%$

- Baseline ferritin levels (ug/mL) (mean (SD): 2257 (748)

- Previous iron chelation: not reported

- LIC (mg/g) mean (SD) (range): 17.1 (9.1) (4.9 - 33.6) N = 16

- Splenectomy n (\%): 11 (61)

- QoL mean (SD): not reported

- Hb, gL mean (SD) (range): 68 (5) (55 - 75)

Inclusion criteria: participants were 10 years or older with a diagnosis of thalassaemia major undergoing iron chelation therapy with subcutaneous DFO, with a SF value between $1000-4000 \mu \mathrm{g} / \mathrm{L}$ over the previous year

Exclusion criteria: not reported

Interventions

DFO: 20 - $60 \mathrm{mg} / \mathrm{kg} /$ day subcutaneously on 5 - 7 days a week (mean (SD) dose at baseline: $34.8(8.9) \mathrm{mg} / \mathrm{kg} /$ day and at end of trial: $37.8(8.9) \mathrm{mg} / \mathrm{kg} /$ day) $)$

DFO/DFP: DFO 20 - $60 \mathrm{mg} / \mathrm{kg} /$ day subcutaneously on 2 days a week (mean (SD) dose DFO for the 29 participants who completed the trial at baseline: $36.0(5.8) \mathrm{mg} / \mathrm{kg} /$ day and at end of trial: 33.3 (6.64) mg/kg/day) with DFP $25 \mathrm{mg} / \mathrm{kg} /$ body weight $3 \mathrm{x}$ daily for 5 days a week)

Outcomes

\author{
Adherence see compliance below \\ Trial-reported outcomes \\ 1. SF change at 1 year \\ 2. LIC (measured by SQUID) change at 1 year \\ 3. ALT \\ 4. FBC \\ 5. Zinc levels
}




\begin{tabular}{|c|c|}
\hline & $\begin{array}{l}\text { 6. AEs } \\
\text { 7. Participant compliance: compliance with DFP was assessed by pill counts, diary } \\
\text { cards and an electronic cap that recorded the time and date of each opening of the } \\
\text { tablet container. Compliance with DFO was assessed by diary cards, weekly physical } \\
\text { examination of infusion sites, and by the Crono }{ }^{\mathrm{TM}} \text { infusion pump that recorded the } \\
\text { number of completed infusions } \\
\text { Primary outcome: not identified }\end{array}$ \\
\hline Identification & $\begin{array}{l}\text { Source of funding: Apotex Research Inc, Toronto, Canada. The last author of the study } \\
\text { is an Apotex employee }\end{array}$ \\
\hline Notes & $\begin{array}{l}\text { The trial inferred that participants had previously received DFO treatment but no details } \\
\text { as to dose, schedule or duration were reported } \\
\text { Sample-size calculation not reported }\end{array}$ \\
\hline
\end{tabular}

\section{Risk of bias}

\begin{tabular}{l|l|l} 
Bias & Authors' judgement & Support for judgement \\
\hline $\begin{array}{l}\text { Random sequence generation (selection } \\
\text { bias) }\end{array}$ & Unclear risk & $\begin{array}{l}\text { The authors did not report any information } \\
\text { about how randomisation was undertaken }\end{array}$ \\
\hline Allocation concealment (selection bias) & Unclear risk & $\begin{array}{l}\text { The authors did not report any informa- } \\
\text { tion about how treatment allocation was } \\
\text { concealed }\end{array}$ \\
\hline
\end{tabular}

Blinding of participants and personnel Unclear risk (performance bias)

jective outcomes
All outcomes except mortality or other ob-

The authors did not report any information as to whether participants, personnel or outcome assessors were blinded to treatment allocation

The authors did not report any information as to whether outcome assessors were blinded to treatment allocation

Although 1 participant in the treatment group was withdrawn due to intolerance to DFP, this is unlikely to effect the findings of the trial

Selective reporting (reporting bias) Unclear risk

Compliance to DFP was pre-specified as an outcome but was not measured or reported in the manuscript

Other bias

Low risk
The trial appears to be free of other sources of bias 
Methods
Study design: single-centre RCT

Study grouping: parallel group

Trial duration: September 2014 to September 2015

Participants

\section{Baseline characteristics}

DFX

- Total \# of participants: 30

- Age mean (SD): 8.9 (2.2)

- Sex male/female: $9 / 21$

- Thalassaemia genotype (\%): $\beta$-thalassaemia major: $100 \%$

- Baseline ferritin levels (ng/mL) median (range): 3216 (2100 - 5862)

- Previous iron chelation: $100 \%$

- Duration of any iron chelation: not reported

- LIC (mg/g): not reported

- Splenectomy n (\%): 4 (13.3)

- QoL mean (SD): not reported

- $\mathrm{Hb}, \mathrm{g} / \mathrm{dL}$ mean (SD): 85 (12)

\section{DFO}

- Total \# of participants: 30

- Age mean (SD): 9.7 (1.9)

- Sex male/female: $10 / 20$

- Thalassaemia genotype (\%): $\beta$-thalassaemia major: $100 \%$

- Baseline ferritin levels $(\mathrm{ng} / \mathrm{mL})$ median (range): 2773 (1980 - 4884)

- Previous iron chelation: $100 \%$

- Duration of any iron chelation: not reported

- LIC (mg/g): not reported

- Splenectomy n (\%): 17 (56.7)

- QoL mean (SD): not reported

- $\mathrm{Hb}, \mathrm{g} / \mathrm{dL}$ mean (SD): $7.9(2.4)$

Inclusion criteria: transfusion-dependent $\beta$-thalassaemia major, ages were $\geq 6$ years, and they had SF levels greater than $1500 \mu \mathrm{g} / \mathrm{L}$ and were on irregular subcutaneous DFO chelation therapy

Exclusion criteria: serum creatinine above the upper age-related normal range, significant proteinuria (urinary protein/creatinine ratio 1.0 in a non-first-void urine sample at baseline), elevated ALT more than 3-fold of the ULN, GI diseases, clinically relevant auditory and/or ocular toxicity related to iron chelation therapy, cardiac disease, and/or SAEs with DFO or DFX, and absolute heutrophilic count $1500 / \mathrm{mm} 3$ or platelet count 100,000/mm3

Pre-treatment: significant difference between the 2 groups with participants having splenectomy 4 in DFX group compared to 17 in DFO group $(\mathrm{P}=0.001)$, hepatitis $\mathrm{C}$ status 2 in DFX group compared to 11 in DFO group $(\mathrm{P}=0.005)$ and baseline ALT baseline mean of 28.2 in the DFX group compared to 46.1 in the DFO group ( $\mathrm{P}=0$. 001)

Interventions

\section{DFX}

- DFX was administered orally as a single daily dose of $20-40 \mathrm{mg} / \mathrm{kg} /$ day on an empty stomach after dissolution in water, apple juice, or orange juice to assure adequate bioavailability. Starting dose of DFX was individualized based on the frequency of blood transfusions

\section{DFO}

Interventions for improving adherence to iron chelation therapy in people with sickle cell disease or thalassaemia (Review) 
- DFO was administered at 20 - $50 \mathrm{mg} / \mathrm{kg} /$ day via subcutaneous infusion over 8 10 hours, 5 days per week

7-day washout phase

\begin{tabular}{|c|c|}
\hline Outcomes & $\begin{array}{l}\text { Adherence to iron chelation therapy rates } \\
\text { During the study, we kept records of all dosages administered, all study medications that } \\
\text { were dispensed and returned, and intervals between visits to determine compliance with } \\
\text { the treatment. The patients' parents were instructed to contact the investigator if the } \\
\text { patients were unable to take the study drug as prescribed } \\
\text { Trial-reported outcomes } \\
\text { 1. decrease in the SF level to }<1500 \mu \mathrm{g} / \mathrm{L} \\
\text { 2. Safety of the drugs that were used }\end{array}$ \\
\hline Identification & $\begin{array}{l}\text { Sponsorship source: not stated } \\
\text { Country: Egypt } \\
\text { Setting: out-patient paediatric hematology clinic Al- Hussein University Hospital, Al- } \\
\text { Azhar University, Cairo, Egypt } \\
\text { Comments: no conflict of interest. } \\
\text { Authors name: Dr Omar Atef Tolba } \\
\text { Institution: Cairo University Children's Hospital } \\
\text { Email: omartolba80@yahoo.com } \\
\text { Address: Dr Omar Atef Tolba, Cairo University Children's Hospital, Department } \\
\text { of Pediatrics, Cairo University, Egypt. Tel: }+201222101717,+20233025539 \text {, Fax: } \\
+20233025539 \\
\text { There is no conflict of interest declared }\end{array}$ \\
\hline Notes & Sample-size calculation not reported \\
\hline
\end{tabular}

\section{Risk of bias}

\begin{tabular}{|c|c|c|}
\hline Bias & Authors' judgement & Support for judgement \\
\hline $\begin{array}{l}\text { Random sequence generation (selection } \\
\text { bias) }\end{array}$ & Unclear risk & $\begin{array}{l}\text { Quote: "the patients were randomized in } \\
\text { a } 1: 1 \text { ratio based on permuted blocks to } \\
\text { receive deferasirox (DFX) or deferoxamine } \\
\text { (DFO) for one year." } \\
\text { Judgement comment: it is unclear risk as } \\
\text { there is imbalance in the groups on several } \\
\text { variables }\end{array}$ \\
\hline Allocation concealment (selection bias) & Unclear risk & $\begin{array}{l}\text { Judgement comment: allocation conceal- } \\
\text { ment not described and imbalance between } \\
\text { groups }\end{array}$ \\
\hline
\end{tabular}

Blinding of participants and personnel High risk (performance bias)

All outcomes except mortality or other objective outcomes
Judgement comment: oral tablet versus subcutaneous infusion - unable to blind participants or personnel 
Hassan 2016 (Continued)

Blinding of outcome assessment (detection High risk bias)

All outcomes except mortality
Quote: "During the study, we kept records of all dosages administered, all study medications that were dispensed and returned, and intervals between visits to determine compliance with the treatment." Judgement Comment: Does not state if outcome assessors were blinded. Assessors would be aware the treatment participants were on

Quote: "no discontinuation of drugs or drop-out of follow-up occurred."

All outcomes

Low risk

Selective reporting (reporting bias) High risk

Quote: "Post-treatment levels of ALT and AST were significantly higher in the DFO group ( $\mathrm{p}=0.022, \mathrm{p}=0.020$, respectively) , both drugs have comparable safety profiles, as the adverse effects noted did not reach clinical significance or lead to discontinuation of treatment with either agent. In the light of the comparable efficacy and safety of both agents for the reduction of iron overload, as was reported in the monotherapy of patients with transfusion-dependent thalassaemia $(31,32)$, the oral preparation merits convenience and therefore patient compliance and adherence to treatment regimen that needs to be taken on a long-term basis."

"The oral DFX is recommended due to more convenience to assure adherence to treatment regimen."

Judgement comment: the data within this trial do not provide evidence that DFX assures adherence. Pre-treatment ALT, AST were also higher in the DFO group - and also reflects imbalance in randomisation. Most outcomes vaguely reported (i.e. compliance - not percentages even though did a count and closely monitored). Also not clear if all drug-related AEs reported (i.e. agranulocytosis). Further the evidence is uncertain from this trial that both drugs of comparable efficacy and safety

Small trial $\mathrm{N}=60$ and short-term followup. Sample-size calculation not reported, and single-centre trial 


\section{Methods}

Study design: multicentre RCT

Study grouping: parallel group

Consecutive thalassaemia major participants $(n=275)$ were observed at the 25 SoSTE centres from September 30, 2000 to January 31, 2008

9 participants did not meet inclusion criteria and 53 patients declined to participate. The remaining 213 participants were included; 105 and 108 respectively, were randomly allocated to DFP-DFO sequential treatment or DFP alone (Fig 1). None of the participants were lost to follow-up

Study duration: 5 year follow-up

\section{Participants}

\section{Baseline characteristics} DFP/DFO

- Total \# of participants: 105

- Age: mean (SD): 23 (8.0)

- Sex: N (\%): F: 55 (50.9)

- Thalassaemia genotype (\%): thalassaemia major (100\%)

- Baseline ferritin levels (ng/mL): mean (SD): 1727 (669)

- Previous iron chelation: $\mathrm{N}=105$

- Duration of any iron chelation: not stated

- LIC (mg/g): mean SD: $4.6(2.8)$

- Splenectomy: N (\%): 17 (14.0)

- QoL mean (SD): not reported

- Hb, g/L: mean SD: 99 (10)

DFP

- Total \# of participants: $\mathrm{N}=108$

- Age: mean SD: 23 (7.8)

- Sex: N (\%): F: 66 (61.1)

- Thalassaemia genotype (\%): thalassaemia major (100\%)

- Baseline ferritin levels (ng/mL): mean (SD): 1868 (845)

- Previous iron chelation: $\mathrm{N}=108$

- Duration of any iron chelation: not stated

- LIC (mg/g): mean (SD): 4.0 (2.3)

- Splenectomy: N (\%): 15 (12.7)

- QoL mean (SD): not reported

- $\mathrm{Hb}, \mathrm{g} / \mathrm{L}:$ mean (SD): 98 (10)

Inclusion criteria: thalassaemia major, SF between 800 and 3000 ug/L over 13 years of age

Exclusion criteria: known intolerance treatment, platelet count $100 \times 109 / 1$ or leucocyte count $3.0 \times 109 / 1$, severe liver damage, heart failure

\section{DFP/DFO}

- DFP $75 \mathrm{mg} / \mathrm{kg}$, divided into 3 oral daily doses, for 4 days/week and DFO subcutaneous infusion (8-12 hours) at $50 \mathrm{mg} / \mathrm{kg}$ per day for the remaining 3 days/week DFP

- DFP alone, at the same dosage (75 mg/ $\mathrm{kg}$ divided into 3 oral daily doses), administered 7 days a week

\section{Adherence}

Compliance was assessed by counting the pills in each returned bag of DFP and by 


\begin{tabular}{|c|c|}
\hline & $\begin{array}{l}\text { assessing the number of infusions of DFO registered on the electronic pump } \\
\text { Trial-reported outcomes } \\
\text { 1. Difference between multiple observations of SF concentrations during the } 5 \text {-year } \\
\text { treatment. A correlation between LIC and SF levels has previously been shown in cohort } \\
\text { of people with thalassaemia major treated with DFP (Olivieri et al, 1995) } \\
\text { 2. Survival analysis } \\
\text { 3. AEs } \\
\text { 4. Costs } \\
\text { 5. Multislice-multiecho T2* MRI scan, available since June 2004, was used in a subgroup } \\
\text { of participants to evaluate variations in the iron content of the heart and liver during the } \\
\text { trial }\end{array}$ \\
\hline Identification & $\begin{array}{l}\text { Sponsorship source: Italian Society for the Study of Thalassaemia and } \\
\text { Haemoglobinopathies (SoSTE) } \\
\text { Country: Italy } \\
\text { Setting: } 25 \text { SoSTE centres in Italy } \\
\text { Comments: NCT } 00733811 \\
\text { Authors name: Aurelio Maggio } \\
\text { Institution: A.O.V. Cervello, U.O.C. di Ematologia } \\
\text { Email: aureliomaggio@virgilio.it } \\
\text { Address: A.O.V. Cervello, U.O.C. di Ematologia II,Cervello”, Palermo, Italy }\end{array}$ \\
\hline Notes & $\begin{array}{l}\text { Follow-up was planned for } 5 \text { years; however, because of the beneficial effects, in terms } \\
\text { of SF levels reduction in the sequential DFP-DFO group, observed after the interim } \\
\text { analysis performed at } 31 \text { January } 2008 \text { the trial was stopped before the planned } 5 \text { years of } \\
\text { treatment were completed for all participants years but mean (SD) duration of treatment } \\
\text { was } 2.5(2.2) \text { and } 2.9(2.1) \text { years for DFP and sequential DFP-DFO groups, respectively } \\
\text { Sample-size calculation reported }\end{array}$ \\
\hline
\end{tabular}

\section{Risk of bias}

Bias

Random sequence generation (selection Low risk bias)
Authors' judgement
Low risk

\section{Support for judgement}

Quote: "The randomization sequence was based on a computer-randomized list in permuted blocks of 10 with a 1:1 ratio," Judgement comment: the randomization sequence was based on a computer-randomized list in permuted blocks of 10 with a 1:1 ratio. The sequence was concealed until interventions were assigned. Randomization was performed per each consecutive participant after verification of the exclusion criteria

Quote: "To ensure allocation concealment, treatment was assigned by telephone contact from the coordinating centre" 
Blinding of participants and personnel High risk

Trial was open-label

(performance bias)

All outcomes except mortality or other ob-

jective outcomes

Blinding of outcome assessment (detection Low risk

bias)

All outcomes except mortality

Quote: “All outcome assessments were done under code by physicians blinded to the trial treatment."

Incomplete outcome data (attrition bias) Unclear risk All outcomes

The statistical analysis was based on the 'intention-to-treat' principle. None of the participants were lost to follow-up. However, SF measurements were only complete for all participants in the first year of the trial and decrease substantially thereafter to $\mathrm{n}=$ 32 in the combined group and $n=26$ in the DFP group

\begin{tabular}{l|l} 
Selective reporting (reporting bias) $\quad$ Low risk & All outcomes reported
\end{tabular}

Other bias

Unclear risk

"Only $21(35 \%)$ subjects in the DFP-alone and $12(24 \%)$ in the sequential DFP-DFO group withdrew definitely from the trial (Table V). The mean time for definitive withdrawal was $152 \pm 103$ (days) in DFPalone versus $112 \pm 76$ (days) in the sequential DFP-DFO group respectively." "The planned duration of treatment was 5 years. However, because of the beneficial effects, in terms of serum ferritin levels reduction in the sequential DFP-DFO group, observed after the interim analysis performed at January 31, 2008 the trial was stopped before the planned 5 years of treatment were completed for all patients. Therefore, the mean duration of treatment was $2.5 \pm 2.2$ and 2 . $9 \pm 2.1$ years for DFP and sequential DFPDFO group respectively"

Judgement comment: withdrawal rate is high and the trial stopped early 


\begin{tabular}{|c|c|}
\hline Methods & $\begin{array}{l}\text { 2-arm parallel RCT. } \\
\text { Number of centres: } 1 . \\
\text { Trial dates: not stated. } \\
\text { Duration of treatment: } 1 \text { year. } \\
\text { Follow-up: none. } \\
\text { Trial undertaken: Chronic Care Centre, Beirut, Lebanon. }\end{array}$ \\
\hline Participants & $\begin{array}{l}\text { Number randomised: } 25 \text { (treatment group: } 14 \text {; comparator group: } 11 \text { ) } \\
\text { Number analysed: } 25 \text { (treatment group: } 14 \text {; comparator group: } 11 \text { ) } \\
\beta \text {-thalassaemia participants, severely iron overloaded and previously poorly chelated } \\
\text { Age range: } 12-40 \text { years } \\
\text { Sex: treatment: } 43 \% \text { male, comparator: } 64 \% \text { male } \\
\text { Ethnicity: not stated }\end{array}$ \\
\hline Interventions & $\begin{array}{l}\text { DFO } \\
\text { - DFO by subcutaneous injection, } 40-50 \mathrm{mg} / \mathrm{kg} 8-12 \text { hours a day, } 5-7 \text { days/week } \\
\text { DFP/DFO } \\
\text { - DFP } 75 \mathrm{mg} / \mathrm{kg} / \text { day orally in } 3 \text { divided doses, } 7 \text { days a week, DFO by } \\
\text { subcutaneous injection, daily dose of } 2 \text { g over } 8 \text { - } 12 \text { hours, } 2 \text { days a week }\end{array}$ \\
\hline
\end{tabular}

Outcomes

Adherence see compliance below Trial-reported outcomes

1. Mean serum iron concentration at baseline, $6 \& 12$ months (primary outcome)

2. Number RBC units during the trial

3. Iron excretion at $1 \& 12$ months

4. Hb level measured weekly for 3 months then monthly for 9 months

5. Liver function measured weekly for 3 months then monthly for 9 months

6. Renal function measured weekly for 3 months then monthly for 9 months

7. Side effects

8. Participant compliance: compliance was assessed by the number of vials of DFX or tablets of DFP used. Safety was determined by detailed clinical and laboratory examination. Participants were also asked to complete questionnaires about any side-effects they experienced reported

Sample-size calculation not reported

\section{Risk of bias}

\section{Bias}

Random sequence generation (selection Unclear risk bias)
Authors' judgement

\section{Support for judgement}

The authors did not report any information about how randomisation was undertaken 
Mourad 2003 (Continued)

\begin{tabular}{|c|c|c|}
\hline Allocation concealment (selection bias) & Unclear risk & $\begin{array}{l}\text { The authors did not report any informa- } \\
\text { tion about how treatment allocation was } \\
\text { concealed }\end{array}$ \\
\hline $\begin{array}{l}\text { Blinding of participants and personnel } \\
\text { (performance bias) } \\
\text { All outcomes except mortality or other ob- } \\
\text { jective outcomes }\end{array}$ & Unclear risk & $\begin{array}{l}\text { The authors did not report any information } \\
\text { as to whether participants, personnel were } \\
\text { blinded to treatment allocation }\end{array}$ \\
\hline $\begin{array}{l}\text { Blinding of outcome assessment (detection } \\
\text { bias) } \\
\text { All outcomes except mortality }\end{array}$ & Unclear risk & $\begin{array}{l}\text { The authors did not report any informa- } \\
\text { tion as to whether outcome assessors were } \\
\text { blinded to treatment allocation }\end{array}$ \\
\hline $\begin{array}{l}\text { Incomplete outcome data (attrition bias) } \\
\text { All outcomes }\end{array}$ & Low risk & $\begin{array}{l}\text { All randomised participants were included } \\
\text { in the analysis for all outcomes: there were } \\
\text { no missing outcome data }\end{array}$ \\
\hline Selective reporting (reporting bias) & High risk & $\begin{array}{l}\text { Data for } 2 \text { pre-specified outcomes were not } \\
\text { reported in the paper: iron excretion at } 1 \\
\text { and } 12 \text { months and renal function. Both are } \\
\text { important clinical markers of the efficacy } \\
\text { of iron chelation therapy }\end{array}$ \\
\hline Other bias & Low risk & $\begin{array}{l}\text { The trial appears to be free of other sources } \\
\text { of bias }\end{array}$ \\
\hline
\end{tabular}

\section{Olivieri 1997}

$\begin{array}{ll}\text { Methods } & \text { 2-arm parallel RCT } \\ \text { Number of centres: } 2 \\ \text { Trial dates: November } 1993 \text { - September } 1995 \\ \text { Duration of treatment: analysis undertaken after } 24 \text { months (mean (SD) duration } 33 \\ \text { (1.0) months, range } 24 \text { - } 43 \text { months) } \\ \text { Follow-up: none } \\ \text { Trial undertaken: Hospital Centres in Toronto and Montreal, Canada. These data are } \\ \text { from the Toronto participants only }\end{array}$

Participants

\section{Baseline characteristics}

Number randomised: 64 (DFO: 32; DFP: 32)

Number analysed: 37 (DFO: 18; DFP: 19). The trial reports details for why 6 and 7 participants respectively were not included in the analysis. The remaining participants had not completed 24 months treatment at the time of analysis for this trial report DFP (L1)

- Age: not reported

- Sex: F: 11; M: 14

- Thalassaemia genotype (\%): thalassaemia major: $100 \%$

- Baseline ferritin levels (ng/mL) mean (SD): 2194 (1251)

- Previous iron chelation: not reported 
- Duration of any iron chelation (duration of treatment in this trial - mean (SD) months): 11.0 (4.2) range 2 - 15

- LIC (mg/g): 9.56 (4.77) Range 2.7 - 21.7

- Splenectomy n (\%): not reported

- QoL mean (SD): not reported

- $\mathrm{Hb}, \mathrm{g} / \mathrm{L}$ : not reported

DFO

- Age: not reported

- Sex: F: 11 M: 14

- Thalassaemia genotype (\%): thalassaemia major: $100 \%$

- Baseline ferritin levels (ng/mL) mean (SD): 2089 (048)

- Previous iron chelation: Not reported

- Duration of any iron chelation (duration of treatment in this trial - mean (SD) months): 11.63 (3.26), range 2 - 15 months

- LIC (mg/g): 7.43 (3.59), range 2.4 - 15.7

- Splenectomy n (\%): not reported

- QoL mean (SD): not reported

- $\mathrm{Hb}, \mathrm{g} / \mathrm{L}$ : not reported

Inclusion criteria: diagnosed with homozygous $\beta$-thalassaemia, 10 years of age or older, willing to participate in the trial

\section{Exclusion criteria:}

- refùsal to participate in the screening

- previously treated with DFP

- serious adverse reactions to DFO

- failed to attend $20 \%$ of the visits in the first 3 months of the trial

- receiving other investigational drugs

- past history of malignancy

- medical, psychological or psychiatric risk

- therapy with an investigational drug would be unwise

- were pregnant or breast feeding

- not using a reliable birth control method

Pre-treatment:

- stratified into high (7 mg Fe/g dry weight liver tissue) and low iron-overloaded (7 $\mathrm{mg} \mathrm{Fe} / \mathrm{g} \mathrm{dw}$ ) according to their hepatic iron concentration as assessed either by liver biopsy or a SQUID (or both)

- 8 participants have been withdrawn from the study due to AEs (2), family reasons (1), psychiatric disorder (1), chronic neutropenia prior to starting on DFP (2), bone marrow transplantation (1) and non compliance with the trial protocol (1)

- 25 participants on DFP and 26 participants on DFO have been used in the present analysis.

- Author goes on to report that results of $n=5$ in DFO were not evaluated as there was no compliance data. A further $\mathrm{n}=5$ participants on DFP and $\mathrm{n}=2$ were excluded for the analysis of the correlation between compliance + successful outcome (as measured by LIC) as there were 6 months of data available. Therefore, for the main outcome the actual $\mathrm{N}=39(\mathrm{n}=20$ in DFP and $\mathrm{n}=19$ in DFO 
Olivieri 1997 (Continued)

\begin{tabular}{ll}
\hline Outcomes & $\begin{array}{l}\text { Adherence see adherence below } \\
\text { Trial-reported outcomes }\end{array}$ \\
& $\begin{array}{l}\text { 1. Change in LIC (measured by SQUID or biopsy) between } 12 \text { months prior to ran- } \\
\text { domisation } \& 24 \text { months duration on trial treatment } \\
\text { 2. Adherence to iron chelation therapy rates defined as per cent of doses administered } \\
\text { (number of doses of the iron chelator taken, out of number prescribed), measured for a } \\
\text { minimum of } 3 \text { months }\end{array}$ \\
\hline Identification & $\begin{array}{l}\text { Sponsorship source: no sponsorship stated } \\
\text { Country: Canada }\end{array}$ \\
& $\begin{array}{l}\text { Setting: Transfusion Clinic } \\
\text { Authors name: Nancy Olivieri } \\
\text { Institution: University of Toronto } \\
\text { Source of funding: not stated }\end{array}$ \\
\hline Notes & $\begin{array}{l}\text { Prior exposure to iron chelators: not reported } \\
\text { Abstract publication. Some data from Pope } 1995 \text { thesis included for baseline character- } \\
\text { istics } \\
\text { Sample-size calculation not reported }\end{array}$ \\
\hline
\end{tabular}

Risk of bias

\begin{tabular}{|c|c|c|}
\hline Bias & Authors' judgement & Support for judgement \\
\hline $\begin{array}{l}\text { Random sequence generation (selection } \\
\text { bias) }\end{array}$ & High risk & $\begin{array}{l}\text { Quote: "After stratification patients by LIC } \\
(>7 \mathrm{mg} \mathrm{Fe} / \mathrm{g} ;<7 \mathrm{mg} \mathrm{Fe} / \mathrm{g} \text { ) 'patients were as- } \\
\text { signed by a research pharmacist who did } \\
\text { not know the patients" }\end{array}$ \\
\hline
\end{tabular}

Allocation concealment (selection bias) Unclear risk

The authors did not report any information about how treatment allocation was concealed

Blinding of participants and personnel High risk (performance bias)

1 treatment a pump and 1 treatment a All outcomes except mortality or other obtablet, participants and researchers would jective outcomes not be blinded to treatment

Blinding of outcome assessment (detection Unclear risk bias)

All outcomes except mortality

Incomplete outcome data (attrition bias) High risk All outcomes
The authors did not report any information as to whether outcome assessors were blinded to treatment allocation

The trial analysed data from $58 \%$ of randomised participants. Of the $42 \%$ randomised participants who were not available for outcome analysis:

- $22 \%$ randomised participants had not 
Olivieri 1997 (Continued)

\begin{tabular}{|c|c|c|}
\hline & & $\begin{array}{l}\text { completed the required } 24 \text { months treat- } \\
\text { ment at the time of analysis for this trial } \\
\text { report; } \\
\text { - } 16 \% \text { DFP-treated participants and } 5 \% \\
\text { DFO treated participants were withdrawn } \\
\text { due to treatment induced side effects } \\
\text { This missing data may inappropriately af- } \\
\text { fect the statistical findings of the trial }\end{array}$ \\
\hline Selective reporting (reporting bias) & Low risk & $\begin{array}{l}\text { All outcomes pre-specified were reported in } \\
\text { the manuscript }\end{array}$ \\
\hline Other bias & Unclear risk & $\begin{array}{l}\text { The trial was reported in an abstract, thus } \\
\text { there are few data available to make an as- } \\
\text { sessment of whether the trial was free of } \\
\text { other bias. Trial stopped early by manufac- } \\
\text { turer }\end{array}$ \\
\hline
\end{tabular}

Pennell 2006

Methods

2-arm parallel RCT

Number of centres: 4

Trial dates: December 2002 - March 2005

Duration of treatment: 1 year

Follow-up: outcome data recorded for duration of treatment

Trial undertaken: 4 participating centres in Italy and Greece

Participants

Number randomised: 61 DFO: 32; DFP: 29

Number analysed: variable across outcomes. Minimum and maximum numbers analysed were: treatment group: 30 - 32; comparator group: 27 - 29. Trial reported details as to why data from 1 participant in the treatment group and 2 in the comparator group were withdrawn from treatment

Transfusion-dependent homozygous participants with $\beta$-thalassaemia major Age: mean (SD) treatment group: 26.2 (4.7) years; mean (SD) comparator group: 25.1 (5.8) years

Sex: treatment group: 50\% male; comparator group: $52 \%$ male

Ethnicity: Greek/Italian: treatment group: 18/14; comparator group: 16/13

Interventions

\section{DFO}

- DFO by subcutaneous injection, $50 \mathrm{mg} / \mathrm{kg}$ for 5 or more days a week DFP

- DFP initial dose $75 \mathrm{mg} / \mathrm{kg} /$ day increasing to $100 \mathrm{mg} / \mathrm{kg} /$ day. Mean actual dose: $92 \mathrm{mg} / \mathrm{kg} /$ day

Outcomes

Adherence rates: DFP compliance was measured using the Medication Event Monitoring System device (Aardex, Zug, Switzerland) and calculated as the percent of openings with an interval longer than 4 hours recorded, divided by number of doses prescribed. DFO compliance was calculated as the percentage of completed infusions, as determined by the Crono pumps, divided by the number of infusions prescribed

Interventions for improving adherence to iron chelation therapy in people with sickle cell disease or thalassaemia (Review) 


\begin{tabular}{|c|c|c|}
\hline & \multicolumn{2}{|c|}{$\begin{array}{l}\text { Trial-reported outcomes } \\
\text { 1. Change over } 1 \text { year in myocardial T2* (primary outcome) } \\
\text { 2. Cardiac volumes and function } \\
\text { 3. LIC } \\
\text { 4. SF } \\
\text { 5. ANC } \\
\text { 6. AEs } \\
\text { 7. ALT } \\
\text { 8. Serum zinc levels } \\
\text { 9. Serum creatinine levels }\end{array}$} \\
\hline Identification & \multicolumn{2}{|c|}{ Trial sponsor: Apotex (manufacturer of DFP) } \\
\hline Notes & \multicolumn{2}{|c|}{$\begin{array}{l}\text { Prior exposure to iron chelators: DFO at a mean (SD) dose of } 39(8) \mathrm{mg} / \mathrm{kg} / \text { day for } 5 \text { - } \\
7 \text { days/week } \\
\text { Sample-size calculation reported }\end{array}$} \\
\hline \multicolumn{3}{|l|}{ Risk of bias } \\
\hline Bias & Authors' judgement & Support for judgement \\
\hline $\begin{array}{l}\text { Random sequence generation (selection } \\
\text { bias) }\end{array}$ & Unclear risk & $\begin{array}{l}\text { The authors did not report any information } \\
\text { about how randomisation was undertaken }\end{array}$ \\
\hline Allocation concealment (selection bias) & Unclear risk & $\begin{array}{l}\text { The authors did not report any informa- } \\
\text { tion about whether treatment allocation } \\
\text { was concealed }\end{array}$ \\
\hline $\begin{array}{l}\text { Blinding of participants and personnel } \\
\text { (performance bias) } \\
\text { All outcomes except mortality or other ob- } \\
\text { jective outcomes }\end{array}$ & High risk & $\begin{array}{l}\text { Open label one treatment subcutaneous } \\
\text { and the other oral so not possible to mask } \\
\text { treatments }\end{array}$ \\
\hline $\begin{array}{l}\text { Blinding of outcome assessment (detection } \\
\text { bias) } \\
\text { All outcomes except mortality }\end{array}$ & Low risk & $\begin{array}{l}\text { The primary outcome was independently } \\
\text { measured in a different country (UK) to } \\
\text { where the trial took place and the findings } \\
\text { were not communicated back to the clini- } \\
\text { cians during the course of the trial }\end{array}$ \\
\hline $\begin{array}{l}\text { Incomplete outcome data (attrition bias) } \\
\text { All outcomes }\end{array}$ & Low risk & $\begin{array}{l}\text { All participants were included in the anal- } \\
\text { ysis of the outcomes SF and AEs } \\
\text { Data from } 1 \text { participant in the treatment } \\
\text { (DFO) group were not included in the } \\
\text { analysis of the cardiac outcomes (primary } \\
\text { outcome) and last observation carried for- } \\
\text { ward method was used to accommodate the } \\
\text { missing data from } 3 \text { other participants (1 } \\
\text { treatment group and } 2 \text { from the compara- } \\
\text { tor group) in the cardiac outcomes (pri- }\end{array}$ \\
\hline
\end{tabular}




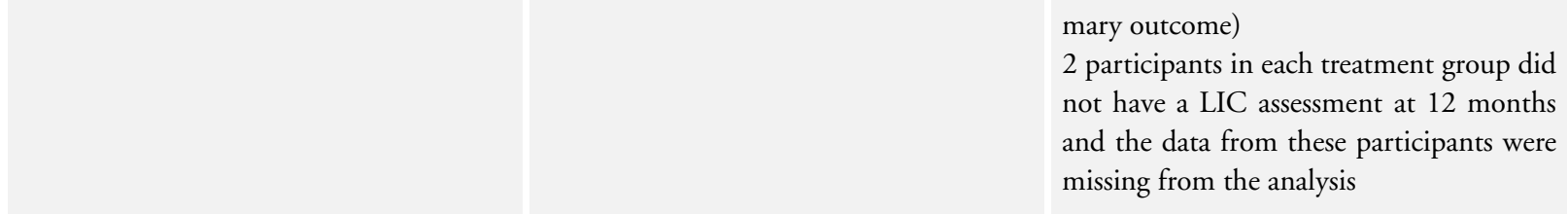

Selective reporting (reporting bias) High risk

The following pre-specified outcomes were not reported in the manuscript: ANC; ALT; serum zinc levels; and serum creatinine levels

Other bias

High risk

There are several imbalances in baseline characteristics between the 2 interventions including a major imbalance in SF measures with the DFO group having much higher levels as well as a greater proportion of participants with severe iron overload (above $2500 \mu \mathrm{g} / \mathrm{L}$ )

Pennell 2014

Methods

Study design: RCT

Study grouping: parallel group

CORDELIA was a prospective, multinational, randomised, open-label, parallel-group, phase 2 trial. A total of $81.2 \%$ of participants $(n=160)$ completed 1 year of treatment

Participants

"Overall, 925 patients were screened and 197 randomized. The majority of patients screened were $\beta$-thalassemia major patients (902/925; 99.1\%). Other patients who were screened and for whom underlying anaemia was captured had low/intermediate 1 myelodysplastic syndrome $(n=4)$, Diamond-Blackfan anaemia, $\beta$-thalassemia intermedia, congenital dyserythropoietic anaemia, and paroxysmal nocturnal haemoglobinuria (all $\mathrm{n}=1$ ). Only $\beta$-thalassemia major patients fulfilled the inclusion criteria and were enrolled in the study. A total of $81.2 \%$ of patients $(n=160)$ completed 1 year of treatment"

\section{Baseline characteristics}

\section{DFX (Exjade)}

- Total \# of participants: 98

- Age mean (SD): 19.9 (6.5)

- Sex (M:F ratio n): 58:40

- Thalassaemia genotype (\%): thalassaemia major: $100 \%$

- Previous iron chelation: DFO: 41 (42.7); DFP: 9 (9.4); DFO + DFP: 21 (21.9);

DFX: 18.1(8.8); Unknown or irregular: 7(7.3)

- Duration of any iron chelation mean (SD) years: $14.0(7.0)$

- LIC (mg Fe/g dw): < 7: 11 (12.1); 7 to < 15: 14 (15.4); $\geq 15: 66$ (72.5)

- Splenectomy n (\%): not reported

- QoL (mean (SD)): not reported

- Median SF (range), ng/mL (per protocol population): 5062 (613 - 15331)

DFO (Desferal) 
- Total \# of participants: 99

- Age mean (SD): 19.7 (6.3)

- Sex (M:F ratio n): 57:42

- Thalassaemia genotype (\%): thalassaemia major: $100 \%$

- Previous iron chelation: DFO: 39 (42.9); DFP: 5 (5.5); DFO + DFP: 219 (23.1) ; DFX: 23 (25.3); Unknown or irregular: 3 (3.3)

- Duration of any iron chelation mean (SD) years: 14.3 (7.2)

- LIC (mg Fe/g dw): 7: 8 (9.9); 7 to $15: 14$ (17.3); $\geq 15: 59$ (72.8)

- Splenectomy n (\%): not reported

- QoL (mean (SD)): not reported

- Median SF (range), ng/mL (per protocol population): 4684 (677 - 13342)

Inclusion criteria: people with $\beta$-thalassemia major, Diamond-Blackfan anaemia, low/ intermediate myelodysplastic syndromes, or sideroblastic anaemia, aged $\geq 10$ years with myocardial T2* $6-20 \mathrm{~ms}, \mathrm{LVEF} \geq 56 \%$, R2 MRI LIC $\geq 3 \mathrm{mg} \mathrm{Fe} / \mathrm{g} \mathrm{dw}$, lifetime history of $\geq 50$ units $\mathrm{RBC}$ transfusions, and receiving $\geq 10$ unit/year of $\mathrm{RBC}$ transfusions Exclusion criteria: participants with serum creatinine above the ULN or significant proteinuria (urinary protein/creatinine ratio $\geq 1.0 \mathrm{mg} / \mathrm{mg}$ in a non-first-void urine sample at baseline; people with ALT $5 \mathrm{x}$ the ULN only if their LIC was $10 \mathrm{mg} \mathrm{Fe} / \mathrm{g} \mathrm{dw}$; considerable impaired GI function or GI disease; history of clinically relevant ocular and/or auditory toxicity related to iron chelation; therapy, and history of HIV seropositivity or malignancy within the past 5 years; clinical symptoms of cardiac dysfunction (shortness of breath at rest or exertion, orthopnoea, exercise intolerance, lower-extremity edema, arrhythmias)

Interventions

DFX (Exjade)

- Once-daily DFX starting dose was $20 \mathrm{mg} / \mathrm{kg}$ per day for 2 weeks, followed by 30 $\mathrm{mg} / \mathrm{kg}$ per day for 1 week, and then continued with $40 \mathrm{mg} / \mathrm{kg}$ per day

DFO (Desferal)

- An intensified dosing regimen of DFO was administered at 50 to $60 \mathrm{mg} / \mathrm{kg}$ per day via subcutaneous infusion over 8 - 12 hours, 5 - 7 days a week, in accordance with Thalassaemia International Federation Guidelines

Mean actual dose over 1-year treatment was $36.764 .2 \mathrm{mg} / \mathrm{kg}$ per day DFX (range, 19. 7- $43.3 \mathrm{mg} / \mathrm{kg}$ per day). Mean actual dose of DFO was $41.568 .7(13.2-60.2) \mathrm{mg} / \mathrm{kg}$ per day, when normalized to a 7-day regimen

Outcomes

Adherence to iron chelation therapy rates: not stated how adherence was measured Trial-reported outcomes

1. Ratio of Gmean myocardial T2* after 1 year of treatment with DFX divided by the ratio of Gmean for DFO

2. Change in LVEF after 1 year of treatment, assessed by absolute change from baseline CMR

3. Absolute change from baseline in LIC after 1-year treatment

4. Absolute change from baseline in SF after 1-year treatment

Identification

Sponsorship source: Novartis Pharma AG

Country: multinational, 11 countries

Setting: 22 centres across 11 countries

Comments: the authors thank Debbi Gorman of Mudskipper Business Ltd for medical editorial assistance. Financial support for medical editorial assistance was provided by 


$\begin{array}{ll} & \text { Novartis Pharmaceuticals } \\ \text { Authors name: Dudley J. Pennell } \\ \text { Institution: National Institute for Health, Research Cardiovascular Biomedical Research } \\ \text { Unit } \\ \text { Email: d.pennell@ic.ac.uk } \\ \text { Address: National Institute for Health Research Cardiovascular Biomedical Research } \\ \text { Unit, Royal Brompton Hospital, Sydney Street, London, SW3 6NP, UK }\end{array}$

\section{Risk of bias}

\begin{tabular}{|c|c|c|}
\hline Bias & Authors' judgement & Support for judgement \\
\hline $\begin{array}{l}\text { Random sequence generation (selection } \\
\text { bias) }\end{array}$ & Low risk & $\begin{array}{l}\text { Quote: " } 22 \text { centers across } 11 \text { countries. Fol- } \\
\text { lowing a } 35 \text {-day screening phase, patients } \\
\text { were randomized in a } 1: 1 \text { ratio" Randomi- } \\
\text { sation was based on permuted blocks; strat- } \\
\text { ification by centre was not conducted }\end{array}$ \\
\hline Allocation concealment (selection bias) & Unclear risk & $\begin{array}{l}\text { Judgement comment: no description of al- } \\
\text { location concealment except that randomi- } \\
\text { sation was based on permuted blocks }\end{array}$ \\
\hline
\end{tabular}

Blinding of participants and personnel High risk (performance bias)

All outcomes except mortality or other objective outcomes
Judgement comment: open-label trial subcutaneous pump versus oral tablet - difficult to blind
Blinding of outcome assessment (detection Low risk bias)

All outcomes except mortality

Incomplete outcome data (attrition bias) Unclear risk All outcomes
Unclear risk
Quote: “Core laboratories were blinded to treatment allocation.In order to eliminate potential unrecognized biases, the core clinical trial team was blinded to the treatment assignment prior to the database lock for the primary analysis."

Judgement comment: 21 withdrawn DFO arm 16 in DFX (78 to 82 completed trial) Efficacy outcomes reported in per protocol and safety in the participants who received the trial drug

Investigator-reported AEs, regardless of causality, were reported in $65(67.7 \%)$ DFX participants and $69(75.8 \%)$ DFO participants (supplemental Table 2). AEs 

curred in $35.4 \%$ of DFX participants and $30.8 \%$ of DFO participants

Judgement comments: It is unclear if investigator-reported AEs and those suspected to be related to trial drug include the same AEs. Also, they only report the end of trial LIC value for the DFX group

Other bias

Low risk

The trial appears to be free of other sources of bias

Taher 2017

Methods

Study design: multicentre RCT conducted in several countries

Study grouping: parallel group

Study duration: 24 weeks

Participants

Baseline characteristics

DFX film-coated tablet

- Total \# of participants: $\mathrm{N}=87$

- Age: 34.6 (19.97)

- Sex: F: 41

- Thalassaemia genotype $\mathrm{N}(\%)$ : thalassaemia major: $70(80.5)$

- Previous iron chelation: 79 (90.8)

- Median SF (range), ng/mL: 2983 (939 - 8250)

- Splenectomy n (\%): not reported

- QoL mean (SD): not reported

- $\mathrm{Hb}, \mathrm{g} / \mathrm{L}$ : not reported

DFX dispersible tablet

- Total \# of participants: $\mathrm{N}=86$

- Age: 35.1 (18.60)

- Sex: F: 47

- Thalassaemia genotype $\mathrm{N}(\%)$ : thalassaemia major: 70 (81.4)

- Baseline ferritin levels (ng/mL) mean (SD): 2089 (048)

- Previous iron chelation: 77 (89.5)

- Median SF (range), ng/mL: 2485 (915 - 8250)

- Splenectomy n (\%): not reported

- QoL mean (SD): not reported

- Hb, g/L: not reported

Inclusion criteria:

- Males and females aged $\geq 10$ years

- Transfusion-dependent thalassaemia and iron overload, requiring DFX dispersible tablet at doses of $\geq 30 \mathrm{mg} / \mathrm{kg} /$ day as per the investigator's decision or participants with very low, low or intermediate (int) risk myelodysplastic syndrome and iron overload, requiring DFX dispersible tablet at doses of $\geq 20 \mathrm{mg} / \mathrm{kg} / \mathrm{day}$ as per the investigator's decision

- History of transfusion of at least 20 PRBC units and anticipated to be transfused

Interventions for improving adherence to iron chelation therapy in people with sickle cell disease or thalassaemia (Review) 
with at least 8 units of PRBCs annually during the study

- $\mathrm{SF}>1000 \mathrm{ng} / \mathrm{mL}$, measured at screening Visit 1 and screening Visit 2 (the mean value will be used for eligibility criteria).

Exclusion criteria:

- Creatinine clearance below the contraindication limit in the locally approved prescribing information. Creatinine clearance will be estimated from serum creatinine at screening Visit 1 and screening Visit 2 and the mean value will be used for eligibility criteria

- Serum creatinine $>1.5 \times$ ULN at screening measured at screening Visit 1 and screening Visit 2 (the mean value will be used for eligibility criteria)

- ALT (SGPT) > 5 x ULN, unless LIC confirmed as >10 mg Fe/dw within 6 months prior to screening visit 1 . Significant proteinuria as indicated by a urinary protein/creatinine ratio $>0.5 \mathrm{mg} / \mathrm{mg}$ in a non-first void urine sample at screening Visit 1 or screening Visit 2

- Participants with significant impaired GI function or GI disease that may significantly alter the absorption of oral DFX (e.g. ulcerative diseases, uncontrolled nausea, vomiting, diarrhoea, malabsorption syndrome, or small bowel resection)

- Liver disease with severity of Child-Pugh Class B or C

Interventions

\section{DFX film-coated tablets}

- DFX film-coated provided as $90 \mathrm{mg}, 180 \mathrm{mg}$ and $360 \mathrm{mg}$ film-coated tablets for oral use

\section{DFX dispersible tablet}

- DFX dispersible tablet provided as $125 \mathrm{mg}, 250 \mathrm{mg}$ and $500 \mathrm{mg}$ dispersible tablets for oral use

Adherence to iron chelation therapy rates
Compliance with medication as assessed by relative consumed tablet count
Trial-reported outcomes
1. Overall safety of both DFX formulations, measured by frequency and severity of AEs
and changes in laboratory values from baseline to 24 weeks
2. Evaluation of both formulations on selected GI AEs (diarrhoea, constipation, nausea,
vomiting, and abdominal pain) during treatment
3. Estimation of treatment compliance
4. Evaluation of both formulations on participant satisfaction, palatability, and GI symp-
toms using PROs
5. Evaluation of the pharmacokinetics of both formulations
6. Reported \% compliant with upper and lower percentages

Identification

Sponsorship source: Novartis Pharmaceuticals

Country: USA

Comments: NCT02125877

Authors name: Ali Taher

Institution: American University of Beirut Medical Center

Email: ataher@aub.edu.lb

Address: Haematology and Oncology, Department of Internal Medicine, Faculty of Medicine, American University of Beirut Medical Center, Beirut, Lebanon 
Taher 2017 (Continued)

Risk of bias

\begin{tabular}{|c|c|c|}
\hline Bias & Authors' judgement & Support for judgement \\
\hline $\begin{array}{l}\text { Random sequence generation (selection } \\
\text { bias) }\end{array}$ & Unclear risk & $\begin{array}{l}\text { "Randomization was stratified by under- } \\
\text { lying disease and previous chelation treat- } \\
\text { ment." } \\
\text { No clear description of randomisation or if } \\
\text { participants were randomised centrally }\end{array}$ \\
\hline
\end{tabular}

Allocation concealment (selection bias) High risk

Quote: "Post- hoc analyses identified that 23 patients on FCT $(26 \%)$ were started on a dose that was higher than recommended in the protocol compared with 8 patients (9.3\%) on DT (not recognized or reported by the investigators as dosing error)."

Judgement comment: the trial was open label and most participants had been on 1 or the other of the trial drugs prior to the trial - doses may have corresponded to prior dosing since there was no description of allocation concealment

Blinding of participants and personnel High risk (performance bias)

Judgement comment: open-label

All outcomes except mortality or other ob-

jective outcomes

Blinding of outcome assessment (detection High risk bias)

All outcomes except mortality

Incomplete outcome data (attrition bias) Unclear risk All outcomes
No description of how outcome assessment was performed - centrally or blinded openlabel trial

Quote: “Overall, all patients were satisfied with their medicine during the study period; satisfaction scores were higher with deferasirox FCT compared with DT at all visits."

Judgement comment: no data provided on number of participants or scores just general statements

Selective reporting (reporting bias) High risk
Quote: "patients discontinued treatment because of AEs $(n=10)$, protocol deviation $(\mathrm{n}=5)$, withdrawal of consent ( $\mathrm{n}$ $=3)$, patient guardian decision $(\mathrm{n}=2)$ , and other reasons (administrative problems, death, and physician's decision, $\mathrm{n}=1$ each)." 
Taher 2017 (Continued)

Judgement comment: investigators do not report all outcomes by treatment assignment, and AEs and SAEs are reported as suspected relationship to trial drug and occurring in > or equal to $10 \%$

Other bias Unclear risk

"The absolute reduction in median serum ferritin (range) in patients receiving FCT was $-350(-4440-3572) \mathrm{ng} / \mathrm{mL}$ and in those receiving DT was -85.5 (-2146-8250) ng/ $\mathrm{mL}$ ); these correspond to a relative change of $-14.0 \%$ with FCT and $-4.1 \%$ with DT. "

Judgement comment: some of difference in change could be accounted for more participants starting on a higher dose of filmcoated tablet

Tanner 2007

Methods

Participants 2-arm parallel RCT

Number of centres: multicentre (12 centres)

Duration of treatment: 12 months

Follow-up: not stated

Trial undertaken: thalassaemia out-patient clinics in Sardinia

Number randomised: 65 (treatment group: 33; comparator group: 32)

Number analysed: not reported

Number completing treatment: 60 (treatment group: 32; comparator group: 28). The reason for the withdrawal was not fully reported by the trial authors

Participants aged 18 years or older with a diagnosis of $\beta$-thalassaemia, currently maintained on subcutaneous DFO and with a myocardial T2* between $8-20 \mathrm{~ms}$

Age: treatment group: mean (SD) 28.7 (5.3) years; comparator group: mean (SD) 28.8

(4.2) years Age range for both arms was 18 - 42 years

Sex: treatment group: 39\% male; comparator group: $44 \%$ male

Ethnicity: not stated

\section{DFO}

- DFO 40 - $50 \mathrm{mg} / \mathrm{kg}$ subcutaneously for 5 days a week (DFO actual dose: 43.4 $\mathrm{mg} / \mathrm{kg}$ for 5 days) with an oral placebo (no further details reported)

DFO/DFP

- DFO 40 - $50 \mathrm{mg} / \mathrm{kg}$ subcutaneously for 5 days a week (DFO actual dose: 34.9 $\mathrm{mg} / \mathrm{kg}$ for 5 days) with DFP $75 \mathrm{mg} / \mathrm{kg}$ daily for 7 days a week

\author{
Adherence see compliance below \\ Trial-reported outcomes \\ 1. Change over 1 year in myocardial T2* (primary outcome) \\ 2. Change in liver T2* at 12 months \\ 3. SF
}




\begin{tabular}{|c|c|}
\hline & $\begin{array}{l}\text { 4. Left ventricular volume \& function } \\
\text { 5. Brachial artery reactivity as a marker of heart failure } \\
\text { 6. Participant compliance with chelation treatments: DFO compliance was calculated } \\
\text { as the percentage of completed infusions, as determined by the Crono pumps, divided } \\
\text { by the number of infusions prescribed. DFP/placebo compliance was measured through } \\
\text { pill counting at the bi-monthly visits } \\
\text { 7. AEs } \\
\text { 8. BNP test }\end{array}$ \\
\hline Identification & $\begin{array}{l}\text { Source of funding: CORDA, Royal Brompton \& Harefield Hospitals Charitable funds, } \\
\text { Cooley's Anemia Foundation, Apotex, UK Thalassaemia Society, University College } \\
\text { London Special trustees Chairty }\end{array}$ \\
\hline Notes & $\begin{array}{l}\text { Prior exposure to iron chelation: DFO mean (SD) dose } 36.4(11.1) \mathrm{mg} / \mathrm{kg} \text { per day for } \\
5.5 \text { day/week (equivalent to } 40.5 \mathrm{mg} / \mathrm{kg} \text { for } 5 \text { day/week). Participants were excluded if } \\
\text { they had previously received DFP } \\
\text { Sample-size calculation reported }\end{array}$ \\
\hline
\end{tabular}

\section{Risk of bias}

\begin{tabular}{l|l|l}
\hline Bias & Authors' judgement & Support for judgement \\
\hline $\begin{array}{l}\text { Random sequence generation (selection } \\
\text { bias) }\end{array}$ & Unclear risk & $\begin{array}{l}\text { The authors did not report any information } \\
\text { about how randomisation was undertaken }\end{array}$ \\
\hline Allocation concealment (selection bias) & High risk & $\begin{array}{l}\text { Trial reports that the participants and clin- } \\
\text { icians were aware of how treatment was to } \\
\text { be allocated }\end{array}$ \\
\hline
\end{tabular}

Blinding of participants and personnel Unclear risk (performance bias)

All outcomes except mortality or other objective outcomes
The authors did not report any information as to whether participants or personnel were blinded to treatment allocation
Blinding of outcome assessment (detection Unclear risk bias)

All outcomes except mortality

Incomplete outcome data (attrition bias) Unclear risk All outcomes
The authors did not report any information as to whether outcome assessors were blinded to treatment allocation

As the trial does not report the number of participants included in each outcome assessment. The trial reports the number completing treatment and the reasons why 3 participants in the treatment group ( 1 adverse event $\& 2$ participant requests) and 4 participants in the comparator group $(3 \mathrm{ad}-$ verse events $\& 1$ participant request) were withdrawn from the trial 
Tanner 2007 (Continued)

\begin{tabular}{|c|c|c|}
\hline Selective reporting (reporting bias) & Low risk & $\begin{array}{l}\text { All outcomes pre-specified were reported in } \\
\text { the manuscript }\end{array}$ \\
\hline Other bias & Low risk & $\begin{array}{l}\text { The trial appears to be free of other sources } \\
\text { of bias }\end{array}$ \\
\hline
\end{tabular}

Vichinsky 2007

Methods

Study design: RCT

Study grouping: parallel group

The study duration was 52 weeks. Participants were recruited by investigators at 44 sites in the USA, France, Italy, UK and Canada

Participants

\section{Baseline characteristics}

DFX

- Total \# of participants: 132

- Age: 15 range $3-54$

- Sex (female \%): 60.6

- Sickle cell genotype N (\%): 100

- Baseline ferritin levels (ng/mL) median (min - max): 3460 (1082 - 1201)

- Previous iron chelation \%: 62.9

- Splenectomy n (\%): not reported

- QoL mean (SD): not reported

\section{DFO}

- Total \# of participants: 63

- Age: 16. Range 3- 51

- Sex (female \%): 55.6

- Sickle cell genotype N (\%): 100

- Baseline ferritin levels (ng/mL) median (min - max): 2834 (1015 - 15578)

- Previous iron chelation \%: 60.3

- Splenectomy n (\%): not reported

- QoL (mean (SD)): not reported

Age group (\% DFX, DFO)

$<6$ years: $3.0,4.8$

6 to $<12$ years: $22.7,23.8$

12 to $<16$ years: $25.0,20.6$

16 to $<50$ years: $47.7,49.2$

50 to $<65$ years: $1.5,1.6$

Inclusion criteria:

- People with $\mathrm{SCD} \geq$ to 2 years of age and with iron overload from repeated blood transfusions

- People receiving regular blood transfusions or those sporadically transfused who received at least 20 units of packed RBCs or equivalent were eligible

- Prior chelation therapy was permitted but was not mandatory

- The serum ferritin level for entry into the screening period of this study was $\geq$ $1000 \mu \mathrm{g} / \mathrm{L}$

Exclusion criteria

Interventions for improving adherence to iron chelation therapy in people with sickle cell disease or thalassaemia (Review) 
Vichinsky 2007 (Continued)

- People were excluded if they had a serum creatinine above the ULN

- Significant proteinuria (as indicated by a urinary protein:creatinine ratio of $\geq 0.5$ confirmed at 2 visits)

- Active hepatitis B or C

- Second and third atrioventricular block, QT interval prolongation, or therapy with digoxin or similar medications

- Treatment with beta blockers or angiotensin-converting enzyme inhibitors was permitted. Those with chelation therapy-associated ocular toxicity were excluded

\section{DFX}

- The initial 24 participants enrolled were randomised to receive DFX $10 \mathrm{mg} / \mathrm{kg}$, all subsequent participants randomised to DFX were dosed at $10-30 \mathrm{mg} / \mathrm{kg}$ according to baseline LIC. DFX was given once daily each morning as a dispersed solution in water, half-an-hour before breakfast. The dose of DFX was reduced by 1 dose level and not re-escalated for participants 15 years and older if serum creatinine increased $33 \%$ above baseline on two consecutive occasions. For children less than 15 years of age, the dose was only decreased if these values were also above the age-appropriate ULN. DFX was interrupted for moderate or severe skin rash and re-instituted at half the initial dose, and dose re-escalation was permitted

DFO

- DFO was administered as a slow subcutaneous infusion over 8 - 12 hours using electronic Microject Chrono infusion pumps on 5 - 7 days a week. In order to facilitate the comparison of different schedules, all DFO doses reported were normalised to administration for 5 days/week (i.e. $50 \mathrm{mg} / \mathrm{kg}$ administered 7 days/week would be reported as $70 \mathrm{mg} / \mathrm{kg}$ )

\section{Adherence to iron chelation therapy rates}

Compliance. For DFX, compliance was assessed by counting the number of tablets returned in bottles at each visit. For DFO, the numbers of vials returned at each visit were counted

\section{Trial-reported outcomes}

\section{Safety assessments}

2. Laboratory assessments were performed at least monthly and included complete blood counts with differential counts. Biochemistry testing included electrolytes, glucose, liver function tests, gamma-glutaryl-transferase, lactate dehydrogenase, cholesterol, triglycerides, uric acid, total protein, C-reactive protein, copper and zinc levels. Iron parameters included total iron, transferrin, transferrin saturation and ferritin. Urinary testing performed on random collections included determination of creatinine, total protein and albumin

3. Physical examinations, ECGs, audiometry and ophthalmological tests were performed at baseline, 12, 24, 36 and 52 weeks. In participants less than 16 years of age, additional assessments included growth velocity and pubertal stage

4. Efficacy assessments. LIC was determined by SQUID biospectrometry at baseline, 24 and 52 weeks. The 24-week assessment was performed primarily for safety purposes, and the change in LIC was calculated between baseline and 52 weeks. SF was assessed monthly during the trial and the change was determined using the baseline and final ferritin level 
Vichinsky 2007 (Continued)

\begin{tabular}{|c|c|c|}
\hline Identification & \multicolumn{2}{|c|}{$\begin{array}{l}\text { Sponsorship source: Novartis Pharmaceuticals } \\
\text { Country: international (Canada, France, Italy, UK and USA) } \\
\text { Setting: medical centre outpatient } \\
\text { Authors name: Elliott Vichinsky } \\
\text { Institution: Children's Hospital and Research Center at Oakland, } \\
\text { Email: evichinsky@mail.cho.org } \\
\text { Address: Children's Hospital and Research Center at Oakland, } 747 \text { 52nd Street, Oak- } \\
\text { land, CA 94609, USA } \\
\text { Novartis Pharmaceuticals Corporation (East Hanover, NJ, USA) co-ordinated the design } \\
\text { and execution of this trial and contributed to the analysis and interpretation of the trial } \\
\text { data. Novartis Pharmaceuticals Corporation also collaborated with the external authors } \\
\text { to assist in the development and approval of the manuscript for publication }\end{array}$} \\
\hline Notes & \multicolumn{2}{|c|}{ Sample-size calculation reported } \\
\hline \multicolumn{3}{|l|}{ Risk of bias } \\
\hline Bias & Authors' judgement & Support for judgement \\
\hline $\begin{array}{l}\text { Random sequence generation (selection } \\
\text { bias) }\end{array}$ & Low risk & $\begin{array}{l}\text { Quote: "The randomisation was per- } \\
\text { formed using an interactive voice response } \\
\text { system" }\end{array}$ \\
\hline Allocation concealment (selection bias) & Unclear risk & $\begin{array}{l}\text { Quote: "stratified according to the follow- } \\
\text { ing age groups: } 2 \text { to }<6 \text { years, } 6 \text { to }< \\
12 \text { years, } 12 \text { to }<16 \text { years and } 16 \text { years } \\
\text { and older. The randomisation sequence in- } \\
\text { cluded permuted block groups of six pa- } \\
\text { tients for each of the three age strata." } \\
\text { Judgement comment: some of the age } \\
\text { groups had few participants and unclear } \\
\text { if allocation would remain concealed with } \\
\text { permuted block groups of } 6 \text { participants }\end{array}$ \\
\hline $\begin{array}{l}\text { Blinding of participants and personnel } \\
\text { (performance bias) } \\
\text { All outcomes except mortality or other ob- } \\
\text { jective outcomes }\end{array}$ & High risk & $\begin{array}{l}\text { Judgement comment: no mention of blind- } \\
\text { ing, but DFO is delivered by infusion } \\
\text { pumps and DFX is a solution in water, so } \\
\text { blinding not feasible }\end{array}$ \\
\hline $\begin{array}{l}\text { Blinding of outcome assessment (detection } \\
\text { bias) } \\
\text { All outcomes except mortality }\end{array}$ & High risk & $\begin{array}{l}\text { Judgement comment: no description of } \\
\text { blinding: Novartis Pharmaceuticals Cor- } \\
\text { poration (East Hanover, NJ, USA) co-or- } \\
\text { dinated the design and execution of this } \\
\text { trial and contributed to the analysis and } \\
\text { interpretation of the trial data. The data } \\
\text { were analysed under supervision of the trial } \\
\text { statistician and were reviewed by the inves- } \\
\text { tigators }\end{array}$ \\
\hline
\end{tabular}

Interventions for improving adherence to iron chelation therapy in people with sickle cell disease or thalassaemia (Review) 
Vichinsky 2007 (Continued)

Incomplete outcome data (attrition bias) Low risk All outcomes
All outcomes reported. 8 participants did not complete and were not included. 6 DFX arm withdraw consent, one in DFO arm. 3 DFO non compliant, 2 DFX and 1 DFO lost to follow-up

\footnotetext{
Selective reporting (reporting bias) Unclear risk

Selective reporting (reporting bias) Unclear risk
}

Other bias

Unclear risk
Quote: "Adverse events, irrespective of the relationship to study medication, which occurred in more than $10 \%$ of patients receiving either treatment, are shown in Table III. As arbitrarily defined by an increased frequency of at least $5 \%$ indicating a potential relationship to drug administration."

Judgement comment: do not report the total number of AEs in all participants, as well there was a substantial number of participants experience SAEs and there is no list of the type except for pain crisis: The number of participants receiving DFX and DFO that reported SAEs was similar (46. $2 \%$ and $42.9 \%$ respectively) and the most common SAE in both groups was sickle cell anaemia with crisis $33.3 \%$ and $31.7 \%$ respectively). Also table of AEs report $\%$ and no totals so impossible to determine total number of participants with an $\mathrm{AE}$

Quote: "The reasons for withdrawal of consent were not included in the database." Quote: "The initial 24 patients enrolled were randomised to receive deferasirox 10 $\mathrm{mg} / \mathrm{kg}$ or deferoxamine at recommended doses of $20-60 \mathrm{mg} / \mathrm{kg}$ based on initial LIC. Subsequently, additional safety information became available for deferasirox suggesting a need to modify the starting dose (Cappellini et al, 2006). Therefore, following the enrolment of the first 24 patients, the study was amended so that all subsequent patients randomised to deferasirox were dosed at $10-30 \mathrm{mg} / \mathrm{kg}$ according to baseline LIC"

Judgement comment: it is important to understand reasons for withdrawals and also the nature of the missing safety information which may have implications for dosing and effects of the dosing amendment 
ADRs: adverse drug reactions

AEs: adverse events

ALT: alanine aminotransferase

ANC: absolute neutrophil count

BNP: brain natriuretic peptide

CBC: complete blood count

CMR: cardiovascular magnetic resonance imaging

DFO: deferoxamine

DFP: deferiprone

DFX: deferasirox

dw: dry weight

ECGs: electrocardiograms

FBC: full blood count

Hb: haemoglobin

HRQoL: health-related quality of life

ICT: iron chelation therapies

IQR: interquartile range

LVEF: left ventricular ejection fraction

LIC: liver iron concentration

MRI: magnetic resonance imaging

PK: pharmacokinetic

PRBC: packed red blood cell

QoL: quality of life

RBCs: red blood cells

RCT: randomised controlled trial

SAEs: serious adverse events

SCr: sickle cell retinopathy

SD: standard deviation

SF: serum ferritin

SGPT: serum glutamate-pyruvate transaminase

SQUID: Superconducting Quantum Interference Device

UIE: urinary iron excretion

ULN: upper limit of normal

WBC: white blood count

Characteristics of excluded studies [ordered by study ID]

\begin{tabular}{ll}
\hline Study & Reason for exclusion \\
\hline Abu 2015 & Wrong study design - qualitative interview questionnaire used \\
\hline Al Kloub 2014 & Wrong study design - qualitative interview questionnaire used \\
\hline Al Kloub 2014a & Wrong study design - cross-sectional study. \\
\hline Al Refaie 1995 & Wrong study design - medication study - not an RCT. \\
\hline Alvarez 2009 & Wrong study design - medication study - not an RCT. \\
\hline $\begin{array}{l}\text { Interventions for improving adherence to iron chelation therapy in people with sickle cell disease or thalassaemia (Review) } \\
\text { Copyright } \odot 2018 \text { The Cochrane Collaboration. Published by John Wiley \& Sons, Ltd. }\end{array}$
\end{tabular}


(Continued)

\begin{tabular}{|c|c|}
\hline Armstrong 2011 & No intervention. \\
\hline Bala 2014 & No intervention. \\
\hline Belgrave 1989 & No intervention. \\
\hline Berkovitch 1995 & Not designed to measure adherence to iron chelation therapy. \\
\hline Chakrabarti 2013 & Not designed to measure adherence to iron chelation therapy. \\
\hline Daar 2010 & Wrong setting - single-centre study. \\
\hline Gomber 2004 & No intervention. \\
\hline Kidson Gerber 2008 & Wrong study design - clinical audit of medication use. \\
\hline Kolnagou 2008 & Wrong study design - medication study not RCT. \\
\hline Leonard 2014 & Wrong study design - single-treatment study. \\
\hline Loiselle 2016 & Review. \\
\hline Mazzone 2009 & Wrong comparator - healthy children not taking iron chelation therapy \\
\hline NCT01709032 & Not designed to measure adherence to iron chelation therapy. \\
\hline NCT01825512 & Not designed to measure adherence to iron chelation therapy. \\
\hline NCT02133560 & Wrong study design - single-centre study with no control. \\
\hline NCT02466555 & Wrong study design - single-centre study with no control. \\
\hline Pakbaz 2004 & Wrong study design - single-centre study with no control. \\
\hline Pakbaz 2005 & Wrong study design - single-centre study with no control. \\
\hline Porter 2009 & Wrong study design - medication intervention not a RCT. \\
\hline Porter 2012 & Wrong study design - medication intervention not a RCT. \\
\hline Vichinsky 2005 & Not designed to measure adherence to iron chelation therapy. \\
\hline Vichinsky 2008 & Not designed to measure adherence to iron chelation therapy. \\
\hline Waheed 2014 & Not designed to measure adherence to iron chelation therapy. \\
\hline Walsh 2014 & Review. \\
\hline
\end{tabular}


(Continued)

Yarali 2006

Not designed to measure adherence to iron chelation therapy.

RCT: randomised controlled trial

Characteristics of studies awaiting assessment [ordered by study ID]

Antmen 2013

\begin{tabular}{|c|c|}
\hline Methods & Prospective cohort study; parallel group \\
\hline Participants & $\begin{array}{l}\text { Participants using DFX - we do not know the disease diagnosis and therefore awaiting classification } \\
\text { Exclusion criteria: not stated }\end{array}$ \\
\hline Interventions & Educational intervention, standard care (as defined in the study) \\
\hline Outcomes & $\begin{array}{l}\text { Exjade Patient Compliance Program (EX-PAT) was established to increase patients' knowledge about DFX usage. } \\
\text { This abstract aimed to represent the results of the pilot EX-PAT program } \\
\text { It is highly recommended to educate the patients under iron chelating treatment about possible complication and } \\
\text { usage of chelating agent }\end{array}$ \\
\hline Notes & $\begin{array}{l}\text { Email sent to author asking for the following information so we could include the study: a full study report of this } \\
\text { abstract? If this is not available would it be possible to have more information on: } 1 \text {. The disease diagnosis of the } \\
\text { participants (were they sickle cell (phenotypes) or thalassaemia (phenotypes) or other); } 2 \text {. How participants were } \\
\text { assigned to intervention or control; } 3 \text {. Any inclusion/exclusion criteria; } 4 \text {. Any group differences; } 5 \text {. Is the age range } \\
\text { for the whole group or is it for the intervention group only? If so could we have the age range for the control group; } \\
6 \text {. Baseline and end of study ferritin levels; } 7 \text {. SAEs or any AEs }\end{array}$ \\
\hline
\end{tabular}

NCT00004982

\begin{tabular}{|c|c|}
\hline Methods & RCT; parallel group \\
\hline Participants & $\begin{array}{l}\text { Inclusion criteria: ages eligible for trial: } 7 \text { years and older (child, adult, senior); genders eligible for study: both } \\
\text { Exclusion criteria: overt cardiac disease }\end{array}$ \\
\hline Interventions & Combination iron chelation therapy, standard care (as defined in the trial) \\
\hline Outcomes & $\begin{array}{l}\text { This small trial is testing the premise that a combination of drugs as a new approach to iron chelation therapy may } \\
\text { reduce side effects and increase efficacy. If both drugs can be given orally, there may be a better chance of finding a } \\
\text { suitable alternative to Desferal. Several combinations of experimental iron chelating drugs are being used in this trial }\end{array}$ \\
\hline Notes & $\begin{array}{l}\text { This trial has been completed. Sponsor: National Institute of Diabetes and Digestive and Kidney Diseases (NIDDK) } \\
\text {. No study results posted } \\
\text { NCT00004982: scant information about the trial was documented on the clinicaltrials.gov web site. We have been } \\
\text { unable to identify any publications from this trial and despite repeated emails to the trial co-ordinator and searching } \\
\text { the funders web site, we have been unable to identify any further details about the trial. Start date: December 1998; }\end{array}$ \\
\hline
\end{tabular}

Interventions for improving adherence to iron chelation therapy in people with sickle cell disease or thalassaemia (Review)

Copyright ( 2018 The Cochrane Collaboration. Published by John Wiley \& Sons, Ltd. 


\section{NCT00004982 (Continued)}

AEs: adverse events

DFX: deferasirox

RCT: randomised controlled trial

SAEs: serious adverse events

\section{Characteristics of ongoing studies [ordered by study ID]}

\section{EudraCT 2012-000353-31}

Trial name or title

Multicentre, randomised, open-label, non-inferiority active-controlled trial to evaluate the efficacy and safety of DFP compared to DFX in paediatric patients aged from 1 month to less than 18 years of age affected by transfusion-dependent haemoglobinopathies

Methods $\quad$ Randomised trial, parallel group

Participants

1. Children on current treatment with DFO or DFX or DFP in a chronic transfusion program receiving at least $150 \mathrm{~mL} / \mathrm{kg} /$ year of packed RBCs (corresponding approximately to 12 transfusions);

2. For those naive to chelation treatment: participants that have received at least $150 \mathrm{~mL} / \mathrm{kg}$ of packed RBCs (corresponding to approximately 12 transfusions) in a chronic-transfusion program and with SF levels $\geq 800$ $\mathrm{ng} / \mathrm{mL}$;

3. For children aged from 1 month to less than 6 years: known intolerance or contraindication to DFO;

4. Written informed consent and patient's informed assent to child's maturity and understanding

Interventions $\quad$ DFP compared to DFX

Outcomes

Percentage of successfully chelated children assessed by SF levels (all participants) and cardiac MRI T2* (children above 10 years of age able to have an MRI scan without sedation)

1. LIC as measured by MRI in those able to undergo MRI scan without sedation

2. Safety and tolerability assessments

3. QoL

\begin{tabular}{l|l}
\hline Starting date & Not stated \\
\hline Contact information & $\begin{array}{l}\text { Consorzio per le Valutazioni Biologiche e Farmacologiche } \\
\text { via Luigi Porta, 14 } \\
\text { Pavia 27100 Italy } \\
\text { deep.2@deep-project.net }\end{array}$
\end{tabular}

Notes 
Trial name or title To assess compliance, efficacy and satisfaction with two different formulation of deferasirox in people with transfusion-dependent beta-thalassaemia

\begin{tabular}{|c|c|}
\hline Methods & RCT; parallel group \\
\hline Participants & $\begin{array}{l}\text { Inclusion criteria: signing informed consent; male or female aged } \geq 2 \text { years at screening; people with trans- } \\
\text { fusion-dependent thalassaemia major; regular transfusion indicated by a blood requirement } \geq 8 \text { blood trans- } \\
\text { fusions per year at screening } \\
\text { Exclusion criteria: people with mean levels of ALT above } 5 \text {-fold the ULN; people with serum creatinine above } \\
\text { ULN; significant proteinuria as indicated by a urinary protein/creatinine ratio }>0.6(\mathrm{mg} / \mathrm{mg}) \text {; creatinine } \\
\text { clearance } \leq 60 \mathrm{~mL} / \mathrm{min} \text {; chronic hepatitis B infection; active hepatitis C infection; pregnancy or breastfeeding; } \\
\text { non-transfusion dependent thalassaemia }\end{array}$ \\
\hline Interventions & DFX (new formulation Jadenu ${ }^{T M}$ ), DFX $\left(\right.$ Exjade $^{\circledR}$ ) \\
\hline Outcomes & $\begin{array}{l}\text { Participants compliance and satisfaction; } 3 \text { months after drug consumption; designed questionnaire to assess } \\
\text { participant compliance and satisfaction; ferritin serum amount; safety; possible GI side effects, including } \\
\text { diarrhoea, and dermatologic symptoms }\end{array}$ \\
\hline Starting date & 22 December 2015 \\
\hline Contact information & $\begin{array}{l}\text { Vice chancellor of research, Shiaz Univeisity of Medical Sciences } \\
\text { COUNTRY: Iran } \\
\text { SETTING: multicentre (outpatient) } \\
\text { Dr. Sezaneh Haghpanah } \\
\text { INSTITUTION:Hematology Research Center, Nemazee Hospital, Shiraz, Iran } \\
\text { EMAIL: haghpanah@sums.ac.ir } \\
\text { ADDRESS: Dr Sezaneh Haghpan Professor of community medicine Hematology Research Center, Nemazee } \\
\text { Hospital, Zand Street, Shiraz, Ira }\end{array}$ \\
\hline Notes & \\
\hline
\end{tabular}

Madderom 2016

\begin{tabular}{l|l} 
Trial name or title & $\begin{array}{l}\text { A randomised controlled trial studying the effectiveness of group medical appointments on self-efficacy and } \\
\text { adherence in sickle cell disease (TEAM study): study protocol }\end{array}$ \\
\hline Methods & RCT; parallel group \\
\hline Participants & $\begin{array}{l}\text { Inclusion criteria: individuals with homozygous or compound heterozygous SCD } \\
\text { Exclusion criteria: individuals with a first visit to the outpatient clinic, patients who cannot communicate } \\
\text { adequately due to language difficulties and/or hearing problems or patients who have behavioral problems } \\
\text { which will limit group functioning }\end{array}$ \\
\hline
\end{tabular}

Interventions

Group Medical Appointment, Individual Medical Appointment (IMA; care-as-usual)

Interventions for improving adherence to iron chelation therapy in people with sickle cell disease or thalassaemia (Review) 


\begin{tabular}{ll}
\hline Outcomes & Primary and secondary endpoints will be measured at baseline (start of the study), after 1.5 years (after two \\
GMA visits) and after 3 years (after four GMA visits), in both groups. Assessments are performed at the \\
hospital, directly before the outpatient visit and in presence of a psychologist. Primary endpoint: 1. Self- \\
efficacy as measured by the validated Sickle Cell Self- Efficacy Scale; Secondary endpoints; 2. Adherence to \\
prescribed treatment by (paediatric) hematologist; 3. QoL as measured with the validated Pediatric Quality \\
of Life Inventory for children and SF-36 for adults. 4. Emergency visits and hospital admissions for SCD \\
related symptoms and complications. 5. Satisfaction with treating physician and nurse (by visual analogue \\
scale: score 1 - 10); 6. Measurement of costs and effects in the GMA and IMA group by an economic analysis \\
according to Dutch guidelines and with respect to an increase in self- efficacy
\end{tabular}

Starting date The trial opened to recruitment in January 2013 for the children and in September 2015 for the adults and is still ongoing

Contact information Marjon H. Cnossen

INSTITUTION: Department of Pediatric Hematology, Erasmus University Medical Center - Sophia Children's Hospital

EMAIL: m.cnossen@erasmusmc.nl

ADDRESS: Department of Pediatric Hematology, Erasmus University Medical Center - Sophia Children's

Hospital, Wytemaweg 80, PO Box 2060, 3000 CB Rotterdam, The NetherlandsAdditional data

Notes

Trial registration: NTR4750 (NL42182.000.12)

\section{NCT02173951}

Trial name or title An algorithm to start iron chelation in minimally transfused young beta-thalassaemia major patients

\begin{tabular}{l|l}
\hline Methods & RCT; parallel group \\
\hline
\end{tabular}

Participants Inclusion criteria: young individuals with $\beta$-thalassaemia major (diagnosed by HPLC, CBC) who started transfusion therapy who received $5-7$ transfusions or less, aged more than 6 months. Pre-transfusional $\mathrm{Hb}$ should be $>9 \mathrm{~g} / \mathrm{dL}$. Serum ferritin should be $\leq 500 \mathrm{ng} / \mathrm{mL}$, transferrin saturation $\leq 50 \%$

Exclusion criteria: 1 . individuals with $\beta$-thalassaemia intermedia, those with other transfusion-dependent anemias (myelodysplasia, other chronic haemolytic anemias, pure red cell aplasia, aplastic anaemia); 2 . Individuals with levels of ALT > 5 the ULN, serum creatinine > ULN on 2 measurements; 3. Indiviudals with history of agranulocytosis (ANC $<0.5 \times 109 / \mathrm{L}$ ). 4. Non-complaint individuals acknowledged by reviewing the patient's records

Interventions DFP, placebo

Outcomes

Primary outcome measures:

determine the time and number of transfusion units as well as amount of infused iron that will lead to appearance of LPI $>0.2$ or TSAT $>50 \%$, serum ferritin $\geq 500 \mathrm{ng} / \mathrm{mL}$ in the studied thalassaemic patients which warrant start of iron chelation

Time frame: 12 months

To determine the time as well as amount of transfused iron (calculated in $\mathrm{mg}$ iron $/ \mathrm{kg}$ ) at which there is LPI appearance of $>0.2$ as well as TSAT reaching $70 \%$, a serum ferritin $\geq 500$ in order to start iron chelation therapy 
Secondary outcome measures:

Evaluation of safety of early use of iron chelation therapy in terms of drug related AEs or SAEs

Time frame: 12 months

To determine the tolerability and safety of early low dose DFP $50 \mathrm{mg} / \mathrm{kg}$ and effectiveness to postpone or prevent SF from reaching $1000 \mathrm{ng} / \mathrm{mL}$ or LPI $>0.6$ or TSAT $>70 \%$ in comparison to participants not starting chelation therapy

Starting date July 2014

Contact information Amira AM Adly,

INSTITUTION: Pediatric Hematology clinic, Ain Shams University Cairo, Egypt

EMAIL: amiradiabetes@yahoo.com

Notes

\section{NCT02435212}

Trial name or title Study to evaluate treatment compliance, efficacy and safety of an improved deferasirox formulation (granules) in paediatric patients $(2-<18$ years old) with iron overload

\begin{tabular}{|c|c|}
\hline Methods & RCT; parallel group \\
\hline Participants & $\begin{array}{l}\text { Inclusion criteria: written informed consent/assent before any study-specific procedures. Consent will be } \\
\text { obtained from parent(s) or legal guardians. Investigators will also obtain assent of patients according to local } \\
\text { guidelines. Male and female children and adolescents aged } \geq 2 \text { and }<18 \text { years. Any transfusion-dependent } \\
\text { anaemia associated with iron overload requiring iron chelation therapy and with a history of transfusion } \\
\text { of approximately } 20 \text { PRBC units and a treatment goal to reduce iron burden ( } 300 \mathrm{~mL} \mathrm{PRBC} \mathrm{=} 1 \text { unit in } \\
\text { adults whereas } 4 \mathrm{~mL} / \mathrm{kg} \text { PRBC is considered } 1 \text { unit for children). Serum ferritin }>1000 \mathrm{ng} / \mathrm{mL} \text {, measured at } \\
\text { screening visit } 1 \text { and screening visit } 2 \text { (the mean value will be used for eligibility criteria) } \\
\text { Exclusion criteria: creatinine clearance below the contraindication limit in the locally approved prescribing } \\
\text { information. Creatinine clearance will be estimated from serum creatinine (using the Schwartz formula) at } \\
\text { screening visit } 1 \text { and screening visit } 2 \text { and the mean value will be used for eligibility criteria. Serum creatinine } \\
>1.5 \mathrm{x} \text { ULN at screening measured at screening visit } 1 \text { and screening visit } 2 \text { (the mean value will be used } \\
\text { for eligibility criteria). ALT and/or AST }>3.0 \mathrm{x} \text { ULN (Criterion no longer applicable, removed as part of } \\
\text { amendment } 1 \text { ): prior iron chelation therapy. Liver disease with severity of Child-Pugh class B or C. Significant } \\
\text { proteinuria as indicated by a urinary protein/creatinine ratio }>0.5 \mathrm{mg} / \mathrm{mg} \text { in a non-first void urine sample } \\
\text { at screening visit } 1 \text { or screening visit } 2 \text {. Those with significant impaired GI function or GI disease that } \\
\text { may significantly alter the absorption of oral DFX (e.g. ulcerative diseases, uncontrolled nausea, vomiting, } \\
\text { diarrhoea, malabsorption syndrome or small bowel resection }\end{array}$ \\
\hline
\end{tabular}

Interventions $\quad$ DFX granule formulation, DFX DT formulation

Outcomes Primary outcome measures: compliance

Change in SF in iron chelation therapy-naive participants.

Secondary outcome measures: domain scores of treatment satisfaction and palatability over time

Overall safety, as measured by frequency and severity of adverse. This includes active monitoring for renal toxicity; including renal failure, hepatic toxicity; including hepatic failure, and gastrointestinal haemorrhage) 
, and changes in laboratory values from baseline (serum creatinine, creatinine clearance, ALT, AST, RBC and WBC). In addition, vital signs, physical, ophthalmological, audiometric, cardiac, and growth and development evaluations will be assessed.

Rate of dosing instructions deviations ('Compliance', using a questionnaire) .

Pre-dose DFX concentrations in all patients.

Pre-dose PK data from all patients will be analysed to support the assessment of compliance.

Post-dose DFX concentrations between 2 and 4 hours post-dose

Change in SF in iron chelation therapy naive and pre-treated participants

PK/PD relationship to explore exposure-response relationships for measures of safety and effectiveness: serum creatinine change from baseline, notable serum creatinine values, serum creatinine clearance change from baseline and notable serum creatinine clearance categories, SF change from baseline, in relationship to derived PK parameters for pre- and post-dose DFX concentrations.

Assess additional safety, as measured by frequency and severity of adverse for granules during extension phase includes active monitoring for renal toxicity; including renal failure, hepatic toxicity; including hepatic failure, and gastrointestinal haemorrhage), and changes in laboratory values from baseline (serum creatinine, creatinine clearance, ALT, AST, RBC and WBC). In addition, vital signs, physical, ophthalmological, audiometric, and growth and development evaluations will be assessed

\author{
AEs: adverse events \\ ALT: alanine transaminase \\ ANC: absolute neutrophil count \\ AST: aspartate transaminase \\ CBC: complete blood count \\ DFO: deferoxamine \\ DFP: deferiprone \\ DFX: deferasirox \\ DT: dispersible tablet \\ GI: gastrointestinal \\ HPLC: high-performance liquid chromatography \\ LIC: liver iron concentration \\ LPI: labile plasma iron \\ MRI: magnetic resonance imaging \\ PK/PD: pharmacokinetic/pharmacodynamic \\ QoL: quality of life \\ RBCs: red blood cells \\ RCT: randomised controlled trial \\ SAEs: serious adverse events \\ SF: serum ferritin
}


TSAT: transferrin saturation ULN: upper limit of normal WBC: white blood cell 
DATA AND ANALYSES

Comparison 1. DFP versus DFO

\begin{tabular}{|c|c|c|c|c|}
\hline Outcome or subgroup title & $\begin{array}{l}\text { No. of } \\
\text { studies }\end{array}$ & $\begin{array}{c}\text { No. of } \\
\text { participants }\end{array}$ & Statistical method & Effect size \\
\hline $\begin{array}{l}1 \text { Adherence to iron chelation } \\
\text { therapy }(\%, S D)\end{array}$ & 3 & & Mean Difference (IV, Random, 95\% CI) & Subtotals only \\
\hline $\begin{array}{l}2 \text { SAEs (from therapy, disease, } \\
\text { non-adherence) }\end{array}$ & 1 & & Risk Ratio (M-H, Random, 99\% CI) & Totals not selected \\
\hline 2.1 Agranulocytosis & 1 & & Risk Ratio (M-H, Random, 99\% CI) & $0.0[0.0,0.0]$ \\
\hline 3 All-cause mortality & 1 & & Risk Ratio (IV, Random, 95\% CI) & Totals not selected \\
\hline $\begin{array}{l}4 \text { Iron overload: defined as } \\
\text { proportion of participants with } \\
\text { serum ferritin } \geq 800(\mu \mathrm{g} / \mathrm{L})\end{array}$ & 1 & & Risk Ratio (M-H, Fixed, 95\% CI) & Totals not selected \\
\hline 5 Organ damage & 1 & & Risk Ratio (M-H, Random, 95\% CI) & Totals not selected \\
\hline 5.1 Liver damage & 1 & & Risk Ratio (M-H, Random, 95\% CI) & $0.0[0.0,0.0]$ \\
\hline $\begin{array}{l}6 \text { Other AEs related to iron } \\
\text { chelation }\end{array}$ & 3 & & Risk Ratio (IV, Random, 99\% CI) & Subtotals only \\
\hline $\begin{array}{l}\text { 6.1 Risk of leukopenia, } \\
\text { neutropenia and/or } \\
\text { agranulocytosis }\end{array}$ & 3 & 192 & Risk Ratio (IV, Random, 99\% CI) & $3.94[0.44,35.50]$ \\
\hline $\begin{array}{l}\text { 6.2 Risk of pain or swelling in } \\
\text { joints }\end{array}$ & 3 & 192 & Risk Ratio (IV, Random, 99\% CI) & $3.38[0.54,21.31]$ \\
\hline 6.3 Risk of nausea/vomiting & 2 & 132 & Risk Ratio (IV, Random, 99\% CI) & $13.68[0.99,188.88]$ \\
\hline $\begin{array}{l}\text { 6.4 Risk of increased liver } \\
\text { transaminase }\end{array}$ & 1 & 44 & Risk Ratio (IV, Random, 99\% CI) & $1.10[0.03,38.47]$ \\
\hline $\begin{array}{l}\text { 6.5 Local reactions at infusion } \\
\text { site }\end{array}$ & 1 & 88 & Risk Ratio (IV, Random, 99\% CI) & $0.17[0.00,9.12]$ \\
\hline
\end{tabular}

\section{Comparison 2. DFX versus DFO}

\begin{tabular}{|c|c|c|c|c|}
\hline Outcome or subgroup title & $\begin{array}{l}\text { No. of } \\
\text { studies }\end{array}$ & $\begin{array}{c}\text { No. of } \\
\text { participants }\end{array}$ & Statistical method & Effect size \\
\hline $\begin{array}{l}1 \text { Adherence to iron chelation } \\
\text { therapy }(\%, S D)\end{array}$ & 1 & & Mean Difference (IV, Random, 95\% CI) & Totals not selected \\
\hline 2 SAEs & 3 & & Risk Ratio (M-H, Random, 95\% CI) & Totals not selected \\
\hline 2.1 Thalassaemia-related SAEs & 2 & & Risk Ratio (M-H, Random, 95\% CI) & $0.0[0.0,0.0]$ \\
\hline $\begin{array}{l}\text { 2.2 SCD-related SAE - painful } \\
\text { crisis }\end{array}$ & 1 & & Risk Ratio (M-H, Random, 95\% CI) & $0.0[0.0,0.0]$ \\
\hline $\begin{array}{l}\text { 2.3 SCD-related SAEs - other } \\
\text { SCD-related SAEs }\end{array}$ & 1 & & Risk Ratio (M-H, Random, 95\% CI) & $0.0[0.0,0.0]$ \\
\hline $\begin{array}{l}3 \text { All-cause mortality } \\
\text { (thalassaemia) }\end{array}$ & 2 & 240 & Risk Ratio (IV, Random, 95\% CI) & $0.96[0.06,15.06]$ \\
\hline
\end{tabular}

Interventions for improving adherence to iron chelation therapy in people with sickle cell disease or thalassaemia (Review) 


4 Proportion of participants with
iron overload (thalassaemia)
4.1 Iron overload defined by
ferritin 1500 ( $\mu$ g/l) or higher
(Thalassaemia)
4.2 Proportion with severe
iron overload (LIC at least 15
mg/Fe/g dw)
4.3 Myocardial T2* $<10 \mathrm{~ms}$
5ther AEs related to iron
chelation - (thalassaemia)
5.1 Total chelation-related AE
5.2 Gastrointestinal upset
5.3 Rash
5.4 Risk of increased blood
creatinine
5.5 Risk of proteinuria
5.6 Risk of increased ALT
5.7 Risk of increased AST
5.8 Risk of diarrhoea
5.9 Risk of vomiting
6 Total AEs (thalassaemia)
7 Other AEs related to iron
chelation (SCD)
7.1 Risk of increased ALT
7.2 incidence of abdominal
pain
7.3 Risk of pain or swelling in
joints
7.4 Risk of diarrhoea
7.5 Nausea/vomiting

Risk Ratio (IV, Random, 95\% CI)

Risk Ratio (IV, Random, 95\% CI)

Risk Ratio (IV, Random, 95\% CI)

Risk Ratio (IV, Random, 95\% CI)

Risk Ratio (IV, Random, 95\% CI)

Risk Ratio (IV, Random, 95\% CI)

Risk Ratio (IV, Random, 95\% CI)

Risk Ratio (IV, Random, 95\% CI)

Risk Ratio (IV, Random, 95\% CI)

Risk Ratio (IV, Random, 95\% CI)

Risk Ratio (M-H, Random, 99\% CI)

Risk Ratio (M-H, Random, 99\% CI)
Subtotals only

$1.18[0.63,2.20]$

$1.00[0.83,1.20]$

$1.10[0.72,1.70]$

Subtotals only

$1.15[0.76,1.73]$

$3.0[0.66,13.69]$

3.05 [0.98, 9.47]

$3.79[0.83,17.38]$

$2.21[0.59,8.29]$

$5.69[0.70,46.33]$

$5.69[0.70,46.33]$

$5.69[0.70,46.33]$

$6.64[0.35,126.78]$

Totals not selected

Subtotals only

$5.29[0.12,232.98]$

$1.91[0.80,4.58]$

$1.06[0.41,2.76]$

$4.14[0.90,18.92]$

$1.63[0.90,2.94]$

\section{Comparison 3. DFX film-coated tablet versus DFX dispersible tablet}

\begin{tabular}{|c|c|c|c|c|}
\hline Outcome or subgroup title & $\begin{array}{l}\text { No. of } \\
\text { studies }\end{array}$ & $\begin{array}{c}\text { No. of } \\
\text { participants }\end{array}$ & Statistical method & Effect size \\
\hline $\begin{array}{l}1 \text { Adherence to iron chelation } \\
\text { therapy }\end{array}$ & 1 & 173 & Risk Ratio (M-H, Random, 95\% CI) & $1.10[0.99,1.22]$ \\
\hline 2 Incidence of SAEs & 1 & 173 & Risk Ratio (IV, Random, 95\% CI) & $1.22[0.62,2.37]$ \\
\hline 3 All-cause mortality & 1 & 173 & Risk Ratio (M-H, Random, 95\% CI) & $2.97[0.12,71.81]$ \\
\hline $\begin{array}{l}4 \text { Incidence of organ damage } \\
\text { (renal event) }\end{array}$ & 1 & 173 & Risk Ratio (IV, Random, 95\% CI) & $1.25[0.83,1.91]$ \\
\hline $\begin{array}{l}5 \text { Other AEs related to iron } \\
\text { chelation }\end{array}$ & 1 & & Risk Ratio (IV, Random, 99\% CI) & Subtotals only \\
\hline $\begin{array}{l}5.1 \text { Total chelation-related } \\
\text { AEs }\end{array}$ & 1 & 173 & Risk Ratio (IV, Random, 99\% CI) & $0.75[0.52,1.08]$ \\
\hline 5.2 Risk of diarrhoea & 1 & 173 & Risk Ratio (IV, Random, 99\% CI) & $0.70[0.29,1.70]$ \\
\hline
\end{tabular}


5.3 Increased urine protein/

urine creatinine ratio

5.4 incidence of abdominal

pain

5.5 Incidence of nausea

\section{Comparison 4. DFP and DFO versus DFP}

\begin{tabular}{|c|c|c|c|c|}
\hline Outcome or subgroup title & $\begin{array}{l}\text { No. of } \\
\text { studies }\end{array}$ & $\begin{array}{c}\text { No. of } \\
\text { participants }\end{array}$ & Statistical method & Effect size \\
\hline 1 Incidence of SAEs & 1 & 213 & Risk Ratio (M-H, Random, 95\% CI) & $0.15[0.01,2.81]$ \\
\hline 2 All-cause mortality & 2 & 237 & Risk Ratio (IV, Random, 95\% CI) & $0.77[0.18,3.35]$ \\
\hline $\begin{array}{l}3 \text { Incidence of chelation therapy- } \\
\text { related AEs }\end{array}$ & 3 & & Risk Ratio (IV, Random, 99\% CI) & Subtotals only \\
\hline $\begin{array}{l}\text { 3.1 Risk of leukopenia, } \\
\text { neutropenia and/or } \\
\text { agranulocytosis }\end{array}$ & 3 & 280 & Risk Ratio (IV, Random, 99\% CI) & $1.15[0.50,2.62]$ \\
\hline $\begin{array}{l}3.2 \text { Risk of pain or swelling in } \\
\text { joints }\end{array}$ & 2 & 256 & Risk Ratio (IV, Random, 99\% CI) & $0.76[0.31,1.91]$ \\
\hline $\begin{array}{l}\text { 3.3 Risk of gastrointestinal } \\
\text { disturbances }\end{array}$ & 1 & 213 & Risk Ratio (IV, Random, 99\% CI) & $0.45[0.15,1.37]$ \\
\hline $\begin{array}{l}3.4 \text { Risk of increased liver } \\
\text { transaminase }\end{array}$ & 2 & 256 & Risk Ratio (IV, Random, 99\% CI) & $1.02[0.52,1.98]$ \\
\hline 3.5 Nausea/vomiting & 1 & 43 & Risk Ratio (IV, Random, 99\% CI) & $0.55[0.13,2.23]$ \\
\hline
\end{tabular}

\section{Comparison 5. DFP and DFO versus DFO}

\begin{tabular}{|c|c|c|c|c|}
\hline Outcome or subgroup title & $\begin{array}{l}\text { No. of } \\
\text { studies }\end{array}$ & $\begin{array}{c}\text { No. of } \\
\text { participants }\end{array}$ & Statistical method & Effect size \\
\hline $\begin{array}{l}1 \text { Other AEs related to iron } \\
\text { chelation }\end{array}$ & 4 & & Risk Ratio (IV, Random, 99\% CI) & Subtotals only \\
\hline $\begin{array}{l}1.1 \text { Risk of leukopenia, } \\
\text { neutropenia and/or } \\
\text { agranulocytosis }\end{array}$ & 3 & 169 & Risk Ratio (IV, Random, 99\% CI) & $1.18[0.09,15.37]$ \\
\hline $\begin{array}{l}1.2 \text { Risk of pain or swelling in } \\
\text { joints }\end{array}$ & 3 & 135 & Risk Ratio (IV, Random, 99\% CI) & $2.39[0.18,32.31]$ \\
\hline $\begin{array}{l}\text { 1.3 Risk of increased liver } \\
\text { transaminase }\end{array}$ & 2 & 104 & Risk Ratio (IV, Random, 99\% CI) & $3.46[0.45,26.62]$ \\
\hline 1.4 Nausea/vomiting & 4 & 194 & Risk Ratio (IV, Random, 99\% CI) & $3.81[0.84,17.36]$ \\
\hline $\begin{array}{l}1.5 \text { Local reactions at infusion } \\
\text { site }\end{array}$ & 2 & 90 & Risk Ratio (IV, Random, 99\% CI) & $0.18[0.01,3.56]$ \\
\hline
\end{tabular}




\begin{tabular}{|c|c|c|c|c|}
\hline Outcome or subgroup title & $\begin{array}{l}\text { No. of } \\
\text { studies }\end{array}$ & $\begin{array}{c}\text { No. of } \\
\text { participants }\end{array}$ & Statistical method & Effect size \\
\hline $\begin{array}{l}1 \text { Adherence to iron chelation } \\
\text { therapy rates }\end{array}$ & 1 & 96 & Risk Ratio (IV, Random, 95\% CI) & $0.84[0.72,0.99]$ \\
\hline 2 Incidence of SAE & 1 & 96 & Risk Ratio (M-H, Random, 95\% CI) & $1.0[0.06,15.53]$ \\
\hline 3 All-cause mortality & 1 & & Risk Ratio (IV, Random, 95\% CI) & Totals not selected \\
\hline $\begin{array}{l}4 \text { Organ damage (serum creatinine } \\
(\geq 33 \%) \text { above baseline in } 2 \\
\text { consecutive occasions) }\end{array}$ & 1 & 96 & Risk Ratio (M-H, Random, 99\% CI) & $3.0[0.16,56.04]$ \\
\hline $\begin{array}{l}5 \text { Other AEs related to iron } \\
\text { chelation }\end{array}$ & 1 & & Risk Ratio (IV, Random, 99\% CI) & Subtotals only \\
\hline 5.1 one year (study end) & 1 & 96 & Risk Ratio (IV, Random, 99\% CI) & $1.08[0.68,1.71]$ \\
\hline $\begin{array}{l}5.2 \text { Risk of leukopenia, } \\
\text { neutropenia and/or } \\
\text { agranulocytosis }\end{array}$ & 1 & 96 & Risk Ratio (IV, Random, 99\% CI) & $1.67[0.27,10.14]$ \\
\hline $\begin{array}{l}5.3 \text { Risk of pain or swelling in } \\
\text { joints }\end{array}$ & 1 & 96 & Risk Ratio (IV, Random, 99\% CI) & $0.89[0.29,2.77]$ \\
\hline 5.4 Gastrointestinal problems & 1 & 96 & Risk Ratio (IV, Random, 99\% CI) & $0.6[0.18,2.04]$ \\
\hline 5.5 ALT (increase $\geq 3$ folds) & 1 & 96 & Risk Ratio (IV, Random, 99\% CI) & $1.33[0.20,8.88]$ \\
\hline 5.6 Skin rash & 1 & 96 & Risk Ratio (IV, Random, 99\% CI) & $5.0[0.10,261.34]$ \\
\hline
\end{tabular}

\section{ADDITIONAL TABLES}

Table 1. Adherence Measurement and Results Table

\begin{tabular}{|c|c|c|}
\hline STUDY & HOW ADHERENCE MEASURED & RESULTS \\
\hline Aydinok 2007 & $\begin{array}{l}\text { - Drug accounting at each visit (by counting the } \\
\text { returned empty blisters of DFP and used vials of } \\
\text { DFO) } \\
\text { - Trial-specific designed questionnaire completed } \\
\text { by the participants or their legal representative/ } \\
\text { guardian (or both) at quarterly intervals }\end{array}$ & $\begin{array}{l}\text { - Compliance was generally excellent during the } \\
\text { entire trial period } \\
\text { - } 1 \text { participant in the DFP treatment arm who } \\
\text { missed more than } 1 \text { chelation dose per week because } \\
\text { of problems with swallowing }\end{array}$ \\
\hline Badawy 2010 & $\begin{array}{l}\text { - Questionnaire on chelation therapy, reasons for } \\
\text { non-compliance, side effects, life activities, } \\
\text { transfusion regimen }\end{array}$ & $\begin{array}{l}\text { - Group II and group I were more compliant to } \\
\text { chelation therapy but difference was statistically non } \\
\text { significant } \\
\text { - Non-compliant participants (compliance less } \\
\text { than } 50 \% \text { ) showed increase in their SF levels in all } \\
\text { studied groups } \\
\text { - In non-compliant participants the reduction in } \\
\text { SF levels was higher in group I and III than in group } \\
\text { II but difference was statistically non significant }\end{array}$ \\
\hline
\end{tabular}


Table 1. Adherence Measurement and Results Table (Continued)

\begin{tabular}{|c|c|c|}
\hline Bahnasawy 2017 & $\begin{array}{l}\text { - Clinical pharmacist analysed data to detect } \\
\text { unnecessary drug therapy, need for additional drug } \\
\text { therapy, ineffective drug product, dosage too low, } \\
\text { adverse drug reaction, dosage too high, non- } \\
\text { compliance }\end{array}$ & $\begin{array}{l}\text { - All } 24 \text { participants in intervention group had } \\
\text { non-adherence at baseline and } 3 \text { where non-adherent } \\
\text { at end of trial } \\
\text { - No data on control group }\end{array}$ \\
\hline Calvaruso 2015 & $\begin{array}{l}\text { - Counting the number of DFP pills in each } \\
\text { returned bag } \\
\text { - Assessing the number of infusions of DFO } \\
\text { registered on the electronic pump }\end{array}$ & $\begin{array}{l}\text { - DFP compliance rate: } 85 \% \\
\text { - DFO compliance rate: } 76 \%\end{array}$ \\
\hline El Beshlawy 2008 & $\begin{array}{l}\text { - Counting the returned empty blisters of DFP } \\
\text { - Counting used vials of DFO }\end{array}$ & $\begin{array}{l}\text { - } 4 \text { participants with DFO-based regimen } \\
\text { excluded from the trial due to lack of compliance } \\
\text { - Compliance was otherwise excellent during the } \\
\text { entire trial period } \\
\text { - Majority of participants had no problems with } \\
\text { the intake and swallowing of the DFP tablets } \\
\text { - } 80 \% \text { of participants in the combination arm and } \\
76 \% \text { of participants in the DFO monotherapy arm } \\
\text { complained about difficulties in the parenteral use of } \\
\text { DFO or problems to insert a needle }\end{array}$ \\
\hline Elalfy 2015 & $\begin{array}{l}\text { - Counting of returned tablets for the oral } \\
\text { chelators } \\
\text { - Counting vials for DFO } \\
\text { - The percentage of actual dose that patient had } \\
\text { taken in relation to the total prescribed dose was } \\
\text { calculated }\end{array}$ & $\begin{array}{l}\text { - DFP/DFX: } 95 \% \\
\text { - DFP/DFO: } 80 \%\end{array}$ \\
\hline Galanello 2006 & $\begin{array}{l}\text { - DFP assessed by pill counts, diary cards and an } \\
\text { electronic cap that recorded the time and date of each } \\
\text { opening of the tablet container } \\
\text { - DFO assessed by diary cards, weekly physical } \\
\text { examination of infusion sites, and by the Crono }{ }^{\mathrm{TM}} \\
\text { infusion pump that recorded the number of } \\
\text { completed infusions }\end{array}$ & $\begin{array}{l}\text { - DFP/DFO: DFO: } 96.1 \pm 5.0 \text { ( } 29 \text { participants) } \\
\text { - DFP compliance was not reported } \\
\text { - DFO: } 95.7 \pm 5.7 \text { ( } 30 \text { participants })\end{array}$ \\
\hline Hassan 2016 & $\begin{array}{l}\text { - Records of all trial medications that were } \\
\text { dispensed and returned } \\
\text { - Parents were instructed to contact the } \\
\text { investigator if the participant were unable to take the } \\
\text { trial drug as prescribed }\end{array}$ & $\begin{array}{l}\text { - All participants compliant with prescribed doses } \\
\text { - No discontinuation of drugs or dropout of } \\
\text { follow-up occurred }\end{array}$ \\
\hline Maggio 2009 & $\begin{array}{l}\text { - Counting the pills in each returned bag of DFP } \\
\text { - Assessing the number of infusions of DFO } \\
\text { registered on the electronic pump }\end{array}$ & $\begin{array}{l}\text { - DFP-DFO group: DFP: } 92.7 \%(\mathrm{SD} \pm 15.2 \% \text {; } \\
\text { range } 37-100 \%) \text { : DFO: } 70.6 \%(\mathrm{SD} \pm 24.1 \% \text {; range } \\
25-100 \%) \\
\text { - DFP alone participants: } 93.6 \%(\mathrm{SD} \pm 9.7 \% \text {; } \\
\text { range } 56-100 \%)\end{array}$ \\
\hline
\end{tabular}

Interventions for improving adherence to iron chelation therapy in people with sickle cell disease or thalassaemia (Review) 
Table 1. Adherence Measurement and Results Table (Continued)

\begin{tabular}{|c|c|c|}
\hline Mourad 2003 & $\begin{array}{l}\text { - Number of vials of DFX used } \\
\text { - Number of tablets of DFO used }\end{array}$ & $\begin{array}{l}\text { - DFO/DFX group: compliance was excellent } \\
\text { (arbitrarily defined as taking }>90 \% \text { of the } \\
\text { recommended doses) in } 10 \text { participants and good } \\
\text { ( } 75 \% \text { to } 90 \% \text { of recommended doses) in } 1 \text { participant } \\
\text { - DFX alone group: compliance was considered to } \\
\text { be excellent in } 11 \text { patients and good in } 3 \text { participants }\end{array}$ \\
\hline Olivieri 1997 & $\begin{array}{l}\text { - Per cent of doses administered: number of doses } \\
\text { of the iron chelator taken, out of number prescribed } \\
\text { - DFP measured with computerised bottles } \\
\text { - DFO measured using ambulatory pumps } \\
\text { - Measured for a minimum of } 3 \text { months }\end{array}$ & $\begin{array}{l}\text { - DFP: } 94.9 \% \pm 1.1 \% \\
\text { - DFO: } 71.6 \% \pm 3.7 \%\end{array}$ \\
\hline Pennell 2006 & $\begin{array}{l}\text { - DFP: measured using the Medication Event } \\
\text { Monitoring System device calculated as the percent of } \\
\text { openings with an interval longer than } 4 \text { hours } \\
\text { recorded, divided by number of doses prescribed } \\
\text { - DFO: calculated as the percentage of completed } \\
\text { infusions, as determined by the Crono pumps, } \\
\text { divided by the number of infusions prescribed }\end{array}$ & $\begin{array}{l}\text { - DFP: } 94 \% \pm 5.3 \% \\
\text { - DFO: } 93 \% \pm 9.7 \%\end{array}$ \\
\hline Pennell 2014 & - Not stated how adherence was measured & $\begin{array}{l}\text { - DFX: } 99.0 \% \pm 3.5 \% \\
\text { - DFO: } 100.4 \% \pm 10.9 \%\end{array}$ \\
\hline Taher 2017 & - Assessed by relative consumed tablet count & $\begin{array}{l}\text { DT: } 85.3 \% \text { (95\% CI: 81.1, 89.5) } \\
\text { - FCT: } 92.9 \% \text { (95\% CI: 88.8, 97.0) }\end{array}$ \\
\hline Tanner 2007 & $\begin{array}{l}\text { - DFO: calculated as the percentage of completed } \\
\text { infusions, as determined by the Crono pumps, } \\
\text { divided by the number of infusions prescribed } \\
\text { - DFP/placebo: pill counting at the bimonthly } \\
\text { visits }\end{array}$ & $\begin{array}{l}\text { - DFO/placebo: DFO: } 91.4 \pm 2.7 \% \text {; placebo: } 89 \text {. } \\
8 \pm 7.2 \% \text {; } \\
\text { - DFO/DFP: DFO: } 92.6 \pm 2.7 \% \text {; DFP: } 82.4 \pm \\
18.1 \%\end{array}$ \\
\hline Vichinsky 2007 & $\begin{array}{l}\text { - DFX: counting the number of tablets returned } \\
\text { in bottles at each visit } \\
\text { - DFO: counting the numbers of vials returned at } \\
\text { each visit }\end{array}$ & $\begin{array}{l}\text { - Ratios of the administered to intended doses of } \\
\text { therapy were high ( } 1.16 \text { for DFX and } 0.97 \text { for DFO), } \\
\text { indicating high adherence to the prescribed treatment } \\
\text { regimens }\end{array}$ \\
\hline
\end{tabular}

DFO: deferoxamine

DFP: deferiprone

DFX: deferasirox

DT: dispersible tablet

FCT: film-coated tablet

SD: standard deviation

SF: serum ferritin 


\section{CONTRIBUTIONSOFAUTHORS}

- Lise Estcourt: searching; selection of trials; eligibility assessment; content expert, and review content development

- Patricia Fortin: searching; selection of trials; eligibility assessment; data extraction, risk of bias assessment, and review content development.

- Karen Madgwick: selection of trials; eligibility assessment; data extraction, risk of bias assessment, content expert.

- Sally Hopewell: methodological expert and review development.

- Marialena Trivella: statistical and methodological expert and review development

- Sheila Fisher: data extraction, risk of bias assessment, review content development.

\section{DECLARATIONSOF INTEREST}

Lise Estcourt: partly funded by the NIHR Cochrane Programme Grant - Safe and Appropriate Use of Blood Components.

Patricia Fortin: funded by the NIHR Cochrane Programme Grant - Safe and Appropriate Use of Blood Components.

Karen Madgwick: none to declare.

Sally Hopewell: partly funded by the NIHR Cochrane Programme Grant - Safe and Appropriate Use of Blood Components.

Marialena Trivella: partly funded by the NIHR Cochrane Programme Grant - Safe and Appropriate Use of Blood Components.

Sheila Fisher: partly funded by the NIHR Cochrane Programme Grant - Safe and Appropriate Use of Blood Components.

\section{SOURCES OF SUPPORT}

\section{Internal sources}

- NHS Blood and Transplant, Research and Development, UK.

To fund the work of the Systematic Review Initiative (SRI)

\section{External sources}

- National Institute for Health Research (NIHR) Cochrane Programme Grant, UK.

To provide funding for systematic reviewers and methodological support from the Centre for Statistics in Medicine, Oxford

\section{DIFFERENCES BETWEEN PROTOCOLAND REVIEW}

See Fortin 2016.

\section{Confidence intervals}

In most studies we were unable to report total adverse events due to participants having one or more of the listed adverse events. We therefore use the $99 \%$ CI to report estimates of effects in subgroups of adverse events.

\section{Assessment of reporting biases}

We could not assess reporting bias as there were fewer than 10 trials for each comparison

\section{Subgroup analysis}

Due to insufficient data we could not undertake subgroup analyses as planned in the protocol (see below). From the outset, we also reported separately on the SCD trial.

Interventions for improving adherence to iron chelation therapy in people with sickle cell disease or thalassaemia (Review) 
- Age of participant (child (one to 12 years), adolescent (13 to 17 years) adult (18+ years))

- Type of disease (SCD or thalassaemia)

- Route of administration of iron chelating agents (oral, intravenous or subcutaneous)

\section{Sensitivity analysis}

We could not undertake sensitivity analyses due to a lack of data.

\section{INDEX TERMS}

\section{Medical Subject Headings (MeSH)}

*Chelation Therapy; *Patient Compliance; Anemia, Sickle Cell [mortality; *therapy]; Benzoates [therapeutic use]; Deferoxamine [therapeutic use]; Iron Chelating Agents [* therapeutic use]; Iron Overload [etiology; ${ }^{*}$ prevention \& control]; Pyridones [therapeutic use]; Quality of Life; Randomized Controlled Trials as Topic; Triazoles [therapeutic use]; beta-Thalassemia [mortality; ${ }^{*}$ prevention $\&$ control]

\section{MeSH check words}

Adolescent; Adult; Child; Humans 